Rochester Institute of Technology

RIT Scholar Works

Theses

8-21-2019

\title{
Human Auditory Discrimination of Bottlenose Dolphin Signature Whistles Masked by Noise: Investigating Perceptual Strategies for Anthropogenic Noise Pollution
}

\author{
Evan L. Morrison \\ elm3948@rit.edu
}

Follow this and additional works at: https://scholarworks.rit.edu/theses

\section{Recommended Citation}

Morrison, Evan L., "Human Auditory Discrimination of Bottlenose Dolphin Signature Whistles Masked by Noise: Investigating Perceptual Strategies for Anthropogenic Noise Pollution" (2019). Thesis. Rochester Institute of Technology. Accessed from 
ROCHESTER INSTITUTE OF TECHNOLOGY

Human Auditory Discrimination of Bottlenose Dolphin Signature

Whistles Masked by Noise: Investigating Perceptual Strategies for Anthropogenic Noise Pollution

by

Evan L. Morrison

A Thesis Submitted in Partial Fulfillment of the Requirements for the

Degree of

Master of Science in Experimental Psychology

Department of Psychology

College of Liberal Arts

Rochester Institute of Technology

Rochester, NY

21 August 2019 
We approve the thesis of Evan L. Morrison:

Dr. Caroline M. DeLong D Date

Professor, Dept. of Psychology, RIT

Faculty Advisor and Chair of the Thesis Committee

Dr. Kirsten Condry $\quad$ Date Associate Professor, Dept. of Psychology, RIT

Reader

Dr. Stephanie Godleski

Date Assistant Professor, Dept. of Psychology, RIT

Reader 


\section{Acknowledgements}

No scientific study would be possible without the combined brilliance and labor of a diverse community of researchers, and this is no exception. I would first like to thank all of the members of my committee who generously offered their time and knowledge towards the successful completion of this project. Thank you to my advisor, Dr. Caroline DeLong, for your tireless support in helping sculpt this design, scouring endless pages of statistics, and long hours spent on meticulous revisions. You were there every step of the way, keeping me moving regardless of the innumerable challenges that this project posed. Thank you to Dr. Kirsten Condry for your many insights, especially on how to create and process data from an elaborate repeated measures design. Thank you to Dr. Stephanie Godleski for your instrumental advice when it came to developing effective means by which to address my hypotheses, both methodologically and mathematically. This research was supported by the College of Liberal Arts' Student Research Fund and the RIT Department of Psychology.

I could not have completed this study without the assistance of those outside of RIT who have helped me along the way. I would like to thank Dr. Heidi Harley and Moriah Deimeke (New College of Florida) for the dolphin signature whistles that served as my stimuli. Thanks also to Dr. Max Kaplan, Dr. T. Aran Mooney, and Jason Dinh (Woods Hole Oceanographic Institution) for providing samples of boat noise. Their work was supported by a grant from the National Science Foundation's Division of Ocean Sciences (OCE Grant 1536782). Finally, I would like to thank Kenneth Tyler Wilcox for lending both his time and statistical expertise. 


\begin{abstract}
Anthropogenic masking noise in the world's oceans is known to impede many species' ability to perceive acoustic signals, but little research has addressed how this noise pollution affects the detection of bioacoustic signals used for communication. Bottlenose dolphins (Tursiops truncatus) use signature whistles which contain identification information. Past studies have shown that human participants can be used as models for dolphin hearing, but most previous research investigated echolocation. In Experiment 1, human participants were tested on their ability to auditorily discriminate among signature whistles from three dolphins. Participants' performance was nearly errorless $(M=98.8 \%)$. In Experiment 2, participants identified signature whistles masked by five different samples of boat noise utilizing different signal to noise ratios. Participant performance was impacted by signal to noise ratio and the similarity of the whistle and noise frequencies. Participants reported listening to the same primary auditory cue as dolphins are believed to use, frequency contour, which indicates similarities in how both species process these signals. Participants reported only a minor change in strategy between noisepresent and noise-absent trials, potentially indicating that the ideal listening strategy does not change in the presence of noise, despite noise negatively impacting performance. This study may provide insight into the impacts of different types of boat noise on dolphin whistle perception. These findings can be used to generate hypotheses to test in future research with dolphin subjects, in order to create inferences of what dolphins' strategies may be when identifying signature whistles in the presence and absence of boat noise. These findings may have implications in conservation and regulations, as they suggest that anthropogenic noise is likely to cause unique and potentially significant harm to dolphins in the marine environment.
\end{abstract}


Table of Contents

\section{Acknowledgements}

iii

Abstract

iv

Introduction

Dolphin Signature Whistles

Human Visual Perception of Signature Whistles. $9-11$

Human Listening Studies $11-17$

The Effect of Masking Noise on Human Auditory Perception $17-19$

The Effect of Anthropogenic Noise on Dolphins

Current Study $23-25$

Experiment 1 25-34

Method $25-31$

Participants $25-26$

Materials 26-28

Procedure 28-31

Pilot Study.

Experimental Test Sessions 29-31

Training Phase 29-30

Testing Phase $30-31$

Interview 31

Results 31-32

Performance Accuracy 31 Interview Responses 


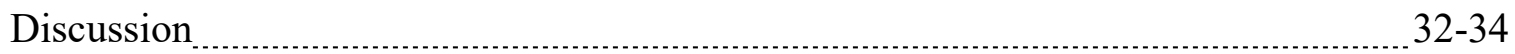

$\begin{array}{lr}\text { Experiment 2 } & \mathbf{3 4 - 5 0}\end{array}$

Method

Participants $\ldots \ldots \ldots$

Materials

Procedure $\quad 37-\ldots$

Pilot Study $\ldots \ldots \ldots$

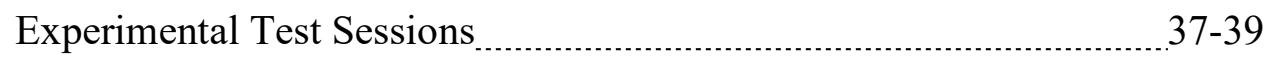

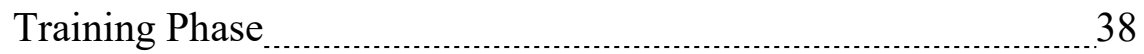

Testing Phase $\ldots \ldots$

Interview

Data Analyses

Results $\ldots \ldots \ldots \ldots \ldots \ldots-\ldots$

Performance Accuracy ........................................................................................ $41-44$

Interview Responses

Strategy Analysis

Discussion $\quad 46-50$

$\begin{array}{lr}\text { General Discussion } & \mathbf{5 0 - 6 1}\end{array}$

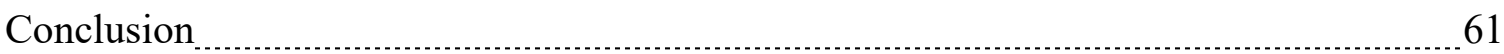

$\begin{array}{lr}\text { References } & 62-71\end{array}$

$\begin{array}{lr}\text { Appendix } & \mathbf{7 2 - 8 2}\end{array}$

$\begin{array}{lr}\text { Tables } & \mathbf{8 3 - 9 6}\end{array}$

$\begin{array}{lr}\text { Figures } & \text { 97-107 }\end{array}$ 
Human Auditory Discrimination of Bottlenose Dolphin Signature Whistles Masked by Noise:

Investigating Perceptual Strategies For Anthropogenic Noise Pollution

Species such as the bottlenose dolphin (Tursiops truncatus) rely heavily on vocalizations for communication and social behavior (Harley, 2008; Sayigh, Esch, Wells, \& Janik, 2007), and utilize biosonar to augment their perception of their environment ( $\mathrm{Au}, 1993)$. There is evidence that boat traffic and noise disrupt dolphin behavior (Lusseau, 2003; Pirotta, Merchant, Thompson, Barton, \& Lusseau, 2015), but it is unclear what the direct impact is of anthropogenic (human-made) noise pollution on dolphins' ability to use vocalizations utilized for communication. A variety of approaches can be implemented to investigate the impacts of noise on dolphin vocalizations, including studies using human participants to model dolphin listeners. Though humans have not previously been utilized as models for dolphin hearing masked by noise, they have proven to be a useful model listener in other contexts, such as investigating properties and perception of echoes from dolphin clicks (e.g., Au \& Martin, 1989; DeLong 2017).

Understanding the potential impacts of anthropogenic noise ranging from large shipping vessels to small recreational watercraft is essential to justify existing measures and regulations to protect these species, as well as establishing new laws. Current regulations apply to behavior that may disrupt or alter the natural behavior of all marine mammals (Marine Mammal Protection Act, 1972), but in order to properly understand what constitutes such a disruption, it is imperative to understand the full scope of human impacts on the cognition, behavior, and perception of marine mammals. Previous research has focused on the basic acoustic properties of noise and dolphin vocalizations (Albuquerque \& Souto, 2013; David, 2006), on the perception of simple tones, broadband clicks, and biosonar echoes in noise (e.g., Au, Moore, \& Pawloski, 
1988; Au \& Penner, 1981; Branstetter et al., 2013b; Weilgart, 2007), or general behavior responses of dolphins to the presence of boats (Janik \& Thompson, 1996; Lusseau, 2003; Pirotta et al., 2015). No studies have yet examined how boat noise directly impacts dolphins' ability to discriminate among their whistle vocalizations.

Prior work has shown that humans are capable of discriminating among dolphin whistlelike sounds and among object echoes ensonified with dolphin clicks (Au \& Martin, 1989; Branstetter, DeLong, Dziedzic, Black, \& Bakhtiari, 2016b; DeLong, 2017; DeLong, Au, Harley, Roitblat, \& Pytka, 2007a; DeLong, Au, \& Stamper, 2007b; DeLong, Heberle, Wisniewski, \& Mercado, 2014; Fish, Johnson, \& Ljungblad, 1976; Gorman \& Sawatari, 1985; Helweg, Roitblat, Nachtigall, Au, \& Irwin, 1995). Such comparisons are possible due to several similarities in human and dolphin hearing. Both species can discriminate between differences in amplitude of 1 dB (Au, 1993; Green, 1993). Likewise, both species have similar frequency discrimination for tonal stimuli within their range of best hearing (Herman \& Arbeit, 1972; Thompson \& Herman, 1975; Wier, Jesteadt, \& Green, 1977). This comparative approach, used by Branstetter and colleagues (2016b), determined that humans, like dolphins, can auditorily discriminate between sounds that simulate dolphin whistles. No research, however, has yet confirmed that humans can successfully discriminate between actual recorded dolphin signature whistles using audition. Prior research has compared the performance of human and dolphin listeners on the same listening task (Au \& Martin, 1989; Branstetter et al., 2016b; DeLong, 2017; DeLong et al., 2007a; DeLong et al., 2007b; DeLong et al., 2014; Gorman \& Sawatari, 1985). Human listeners, used to model dolphin hearing and processing, have been used to better understand the auditory processing of dolphins because humans are able to explain their strategies verbally. When human listeners perform similarly to dolphins on a matching task, making similar errors under similar 
conditions, we can infer that humans and dolphins are likely using the same strategies, leading to the same mistakes. This technique could be used to model the process of whistle discrimination when anthropogenic noise is present. The current study will attempt to investigate human performance and strategies, to lay a foundation for a future study with dolphins which may use a modified version of the same procedure.

First, I will define and discuss signature whistles and expand upon research on discrimination of these whistles by dolphins. I will also review human performance discriminating between dolphin signature whistles visually using spectrograms. Next, I will review the ability of human listeners to discriminate auditorily among echoes produced from dolphin echolocation clicks, as well as their ability to discriminate among whistle-like sounds. I will then explain how masking noise has been found to impact human auditory perception. Finally, I will review anthropogenic noise in the ocean and its effects on dolphin behavior.

\section{Dolphin Signature Whistles}

Many species of dolphins communicate with a variety of vocalizations, including whistles, clicks, and burst pulses (Herzing, 1996; Overstrom, 1983; Sayigh et al., 2007). Knowing the characteristics and uses of these vocalizations is important to grasp how anthropogenic noise may impact many aspects of dolphins' lives, such as inter-individual communication and foraging. Though the present study focuses on communicative vocalizations, many of the following vocalizations are used in multiple situations, including social scenarios. Echolocation clicks are used for navigation, foraging, and object perception, but can also be produced during social interactions or to coordinate foraging efforts (Au, 1993; Benoit-Bird \& Au, 2009a, 2009b). Clicks are directional, short, broad-band sounds, lasting between 50 and 70 $\mu \mathrm{s}$, with peak frequencies between 120 and $130 \mathrm{kHz}(\mathrm{Au}, 1980)$. Some vocalizations are 
associated with communication within specific social and emotional behavioral contexts, such as burst pulses, which are often associated with aggression (Herzing, 1996; Overstrom, 1983). Burst pulses generally $2 \mathrm{~s}$ or less (Overstrom, 1983). Whistles seem to be largely used for communication as well, primarily for group cohesion and coordination, as well as individual identification (Herzing, 1996; Quick \& Janik, 2008). Whistles are continuous, narrow-band, frequency-modulated sounds. Whistles appear to range from 1 to over $24 \mathrm{kHz}$ in frequency, and have an average duration of about $1 \mathrm{~s}$ (Harley, 2008).

Signature whistles are a specific subset of all types of whistles, used to identify individual dolphins in a manner similar to human names (Gridley et al., 2014; Janik \& Sayigh, 2013; Janik, Sayigh, \& Wells, 2006; Janik \& Slater, 1998; Quick \& Janik, 2008, 2012; Sayigh et al., 2007; Sayigh, Tyack, Wells, \& Scott, 1990). The signature whistle hypothesis states that dolphins produce unique, stereotyped whistles, especially when isolated from their conspecifics (Watwood, Tyack, \& Wells, 2004). Simply put, signature whistles are individualized whistles which dolphins seemingly create to refer to themselves (Fripp et al., 2005; Janik, Dehnhardt, \& Todt, 1994; Miksis, Tyack, \& Buck, 2002). Signature whistles are most-often produced by the dolphin to which they refer (Janik \& Sayigh, 2013; Sayigh et al., 2007). For example, dolphin A will most often produce its own signature whistle, but will also occasionally produce the signature whistle of dolphin B or dolphin $\mathrm{C}$. These whistles range in frequency from about 1 to $30 \mathrm{kHz}$ (Sayigh \& Janik, 2010) and have durations of 0.1 to 4 s (Buckstaff, 2004). Spectrograms of signature whistles from three dolphins are displayed in Figures 1, 2, and 3.

Signature whistles vary on several dimensions, including voice cues (sound quality or timbre), duration, amplitude, and frequency contour (Gridley et al., 2014; Harley, 2008; Janik et al., 1994; Janik \& Sayigh, 2013; Janik et al., 2006; Kershenbaum, Sayigh, \& Janik, 2013). Voice 
cues, defined by Sayigh and colleagues (2017) as the manner in which "characteristics of the vocal tract render vocalizations of an individual uniquely identifiable," (p. 1067). However, dolphins do not seem to consider these voice cues when perceiving signature whistles, a trait which may result from voice cues being inconsistent at different depths (Sayigh et al., 2017). Frequency contour has been defined as how the frequency (or multiple harmonic frequencies) of a whistle changes over the duration of the whistle. Past studies indicate that frequency contour may be the most crucial identifying element in a signature whistle for dolphins and humans alike (Harley, 2008; Janik et al., 1994; Janik, King, Sayigh, \& Wells, 2013; Janik et al., 2006; Sayigh et al., 2007). It is also noteworthy that the signature whistles of some dolphins contain long, looping frequency contour patterns, and that a signature whistle emitted on some occasions by the same dolphin may sometimes omit some of these loops, as demonstrated by the signature whistles of Dolphin 3 (see Figure 3).

There is evidence for several uses of signature whistles based on context-driven research. Dolphins generally increase their use of their own signature whistles when separated from their group, which supported the idea that signature whistles are used to maintain group cohesion (Janik \& Slater, 1998; Smolker, Mann, \& Smuts, 1993). Dolphins also use more signature whistles when two groups of dolphins meet in the wild (Quick \& Janik, 2012). This could also be related to cohesion, helping to ensure that all dolphins return to their original group after parting ways. Alternatively, it could be used as a form of greeting or introduction between unfamiliar individuals. Finally, signature whistles are likely involved in social bonding, especially between mothers and calves (King, Sayigh, Wells, Fellner, \& Janik, 2013). Mother and calf pairs are especially likely to use their signature whistles when they become separated, with calves using their signature whistles more often than their mothers (Smolker et al., 1993). Smolker and 
colleagues (1993) also found that the likelihood of using their signature whistle increased with the distance of separation, ranging from just under $50 \%$ of separations under $2 \mathrm{~m}$ involving signature whistles to $100 \%$ of observed separations of $100 \mathrm{~m}$ or more involving signature whistles.

Bottlenose dolphins appear to develop their own unique signature whistle within their first one or two years of life, after which these whistles seem to be stable and unchanging (Fripp et al., 2005; Sayigh et al., 1990). Bottlenose dolphins seem to use elements of familiar signature whistles in their own signature whistle (Fripp et al., 2005; Sayigh et al., 1990). This often means that signature whistles, though distinct from close associates, may resemble slightly more distant relatives or friends (Fripp et al., 2005). Sayigh, Tyack, Wells, Scott, and Irvine (1995) found that the signature whistles of female offspring are usually more distinct from their mothers, while male offspring tend to have signature whistles more similar to their mothers. This was suggested to be due to selective pressure for the females, who were more likely to stay with their mothers as adults, to distinguish themselves from their mothers. Male offspring would have no such selective pressure, as they are less likely to stay with their mothers as adults (Sayigh et al., 1995). Signature whistles seem to be stable across a dolphin's lifetime, once they have been fully developed by a young dolphin (Sayigh et al., 1990). Furthermore, in captivity, dolphins often model their own signature whistles, at least in part, after familiar anthropogenic noise, especially their trainers' whistles (Miksis et al., 2002). Miksis and colleagues (2002) discovered that dolphins that were raised with human trainers developed signature whistles that were more likely to have a relatively flat frequency contour overall, like the sound of a trainer's whistle, and to contain longer segments of continuously flat frequency. This indicates that dolphins do not 
develop their own signature whistles from scratch, but instead model them after familiar sounds and other dolphins' whistles.

Sayigh and colleagues (1999) attempted to determine whether dolphins recognized individual signature whistles by counting the number of head turns towards and away from a submersible speaker which played the various signature whistles to specific dolphins. For mother dolphins, the speaker played either the signature whistle of their own calves or of other familiar calves. For calves, the speaker played the signature whistles of either the calves' mothers, or of a familiar female of similar age to their mother. Dolphins were more likely to turn their heads towards the sound of a signature whistle of their parent or offspring than an unrelated conspecific (Sayigh et al., 1999). This response suggests that these whistles carry identifying information about the individual whose signature whistle is being vocalized, and that other dolphins actively recognize these whistles.

A study by King and Janik (2013) on wild dolphins replayed synthetic versions of signature whistles, which lacked any identifying voice features (whistle characteristics created by individual differences in vocal tracts among dolphins), back to the original dolphin which had produced the signature whistle. On most trials, dolphins responded to calls of their own signature whistle by calling back with the same whistle (their own, in this case). They responded to other, familiar signature whistles with this sort of mimicry only twice in twelve trials. Completely unfamiliar signature whistles seemed to elicit no mimicry of the heard signature whistle. King and Janik (2013) suggested that this might indicate that signature whistles could be used by dolphins to address conspecifics more directly, if hearing its own signature whistle can so heavily draw a bottlenose dolphin's attention. 
Earlier studies observing behavioral responses to the actual signature whistles of family members (Sayigh et al., 1999) and responses to synthetic signature whistles of family members (Janik et al., 2006) found that bottlenose dolphins react strongly to familiar signature whistles whether or not they recognize the voice cues in the vocalization. Voice cues have been defined as additional auditory features, especially timbre, generally created by the unique vocal tracts, especially the phonic lips, of the vocalizing dolphin (Janik et al., 2006). Synthetic whistles were produced by generating sounds which matched real signature whistles in frequency contour and amplitude contour but did not contain any voice cues (Janik et al., 2006). Familiar signature whistles, even synthetic ones, elicited strong behavioral responses in terms of vocalizations (Janik et al., 2006) as well as head turns (Sayigh et al., 1999). Though dolphins seem to respond strongly to any version of a familiar signature whistle, Sayigh, Wells, and Janik (2017) indicate that dolphins do not respond more strongly to any other type of whistle produced by a familiar dolphin than from unfamiliar dolphins. Familiar individuals' vocalizations ought to contain voice cues (e.g., timbre) that could distinguish them from the vocalizations of unfamiliar conspecifics, but these differences do not seem to be salient. Given that dolphins seem able to use frequency contour to identify signature whistles, but not voice cues, it is likely that a signature whistle's meaning is derived primarily, if not solely, by its frequency contour (Branstetter et al., 2016b; Janik et al., 2006; Sayigh et al., 1999; Sayigh et al., 2017). Since frequency contour is so important for a dolphin's understanding or recognition of signature whistles, it is likely that the most detrimental types of anthropogenic noises will be those which mask frequency contour more effectively. If humans, like dolphins, rely upon frequency contour to identify signature whistles, they are more likely to be an effective model listener for investigating dolphin perceptual strategies and abilities. 


\section{Human Visual Perception of Signature Whistles}

Previous studies that have explored human classification of dolphin signature whistles have used visual representations of the whistles, rather than auditory stimuli (Janik, 1999; Janik \& Slater, 1998; Kershenbaum et al., 2013; Sayigh et al., 2007; Watwood et al., 2004). These studies have involved humans looking at and categorizing large numbers of dolphin whistle spectrograms, which are visual, graphical representations of the frequency and amplitude of a sound over time (e.g., Figures 1-3). Participants were expected to categorize many exemplars of dolphin whistles from a small number of dolphins based only on whatever information was visible in the spectrograms, such as duration, amplitude, and frequency contour. In most of these studies, humans worked independently and without additional information on the whistles or individual dolphins, except for the study by Watwood and colleagues (2004), in which Watwood was responsible for the categorization of whistles and was aware of the identity of the dolphins producing each whistle. Humans have consistently performed extremely well at this type of task, achieving nearly 100\% accuracy and even outperforming computer programs (Janik, 1999; Kershenbaum et al., 2013).

Studies which utilize human categorization of signature whistles have primarily been performed with intentions other than achieving understanding of human visual categorization of signature whistles. This research has instead evaluated the efficacy of automated systems on whistle-sorting (Janik, 1999; Kershenbaum et al., 2013) or investigated other research questions, such as analyzing the similarities of dolphin whistles across generations (Sayigh et al., 1995) and testing the signature whistle hypothesis (Janik \& Slater, 1998; Watwood et al., 2004). Many of these studies have had extremely low numbers of human observers, primarily ranging from just one participant (Janik \& Slater, 1998; Watwood et al., 2004) to ten participants (Kershenbaum et 
al., 2013; Sayigh et al., 2007). There is still a need for more research on human discrimination and categorization of dolphin signature whistles, with greater sample sizes, more direct focus on human performance, and analysis of what variables impact that performance. There is still insight to be gained from this area of research about the nature of signature whistles and human perception of these whistles.

Sayigh and colleagues (1995) asked 74 human participants to view spectrograms of dolphin signature whistles and rate their similarities, to compare the similarity of signature whistles among mothers and calves. Rather than identifying individual dolphins' signature whistles, this methodology relied upon participants to assess and rate similarities in frequency contour between different individuals' signature whistles. This methodology enabled the researchers to draw conclusions about the similarities of signature whistles between mothers and calves based on human visual perception of those whistles. On average, participants rated the majority of male calves" signature whistles either "somewhat similar" or "very similar" to their mothers' signature whistles, but rated the majority of female calves signature whistles "not similar" to their mothers'. This study also validated the use of human observation of signature whistle spectrograms as a means of drawing conclusions about the nature of signature whistles. Janik (1999) asked five human observers to categorize simplified line spectrograms of 40 signature whistles and 64 other whistles into groups by similarities and patterns in the spectrograms. Participants were presented with simplified line spectrograms which displayed only the absolute frequency and frequency contour, with no indication of the amplitude or harmonics (simultaneously occurring sounds of lower or higher frequency than the peak frequency, which typically follow the same frequency contour). Three computer systems were also used to attempt to automatically sort the whistles. Human participants were given no 
additional information about the whistles or dolphins that produced them. The whistles came from five dolphins, with several exemplars of each dolphin's signature whistle present in the sample. Participants always identified the five signature whistle types (each produced by a one dolphin individual). Of the 64 non-signature whistles, only 5 were misidentified as signature whistles. Of the 40 signature whistles, 32 were sorted correctly by all five human observers, 7 were correctly sorted by all but one observer, and only one whistle was incorrectly sorted by two observers. Human observers performed significantly better than all three of the computer systems that were tasked with sorting the whistles in the same study. These results demonstrate the reliability with which humans are able to discriminate among dolphin whistles when using visual representations, but these findings do not necessarily predict success in discriminating auditorily among whistles.

Human visual categorization or comparison of signature whistles has been shown to be extremely reliable (Janik, 1999), even when compared against the performance of computer programs (Janik, 1999; Kershenbaum et al., 2013). This seems to be an indication of the presence of noticeable differences between signature whistles, especially in frequency contour, profound enough to be salient to humans. The present study seeks to determine whether these differences will be salient to humans when perceived auditorily instead of visually.

\section{Human Listening Studies}

When researching dolphin auditory processing, humans often serve as a valuable model organism, as humans and dolphins share some similarities in auditory processing, and humans are able to give verbal explanations and feedback on their strategies in auditory discrimination (Au \& Martin, 1989; Branstetter et al., 2016b; DeLong, 2017; DeLong et al., 2007a; DeLong et al., 2007b; DeLong et al., 2014). In these studies, humans and dolphins are often exposed to the 
same auditory stimuli (e.g., echoes). For these studies, the ultrasonic echoes are altered in frequency to suit the hearing range of human listeners. Previous studies have analyzed the ability of dolphins and humans to discriminate among recorded object echoes, which were generated using a simulated dolphin echolocation click (Au \& Martin, 1989; DeLong, 2017; DeLong et al., 2007a; DeLong et al., 2007b; DeLong et al., 2014; Fish, Johnson, \& Ljungblad, 1976).

Branstetter and colleagues (2016b) investigated both species' ability to discriminate sounds similar to natural dolphin whistles. Many of these studies have also involved interviews or questionnaires for the human participants, which have asked participants what elements of the sounds they were attending to when performing the discrimination tasks (Au \& Martin, 1989; Branstetter et al., 2016b; DeLong, 2017; DeLong et al., 2007a; DeLong et al., 2007b; DeLong et al., 2014; Gorman \& Sawatari, 1985). By analyzing human feedback and comparing performance between humans and dolphins (specifically error patterns), researchers have been able to generate informed hypotheses about strategies dolphins may use in their own auditory processing.

Research by Au and Martin (1989) utilized this approach to analyze dolphin biosonar. They used a simulated dolphin echolocation click to produce the echoes for this study, then slowed down the echoes by a factor of 50 for human listeners. Echoes were produced from cylinders made of glass and various metals. A second phase of the study used echoes made from foam spheres and cylinders. Both sets of stimuli had previously been used in echo discrimination tasks designed for dolphins. Participants in these studies reported using duration, frequency contour, and changes over time in the echoes' amplitudes to discriminate between the echoic stimuli. When listening to echoic stimuli, participants typically used frequency contour by noticing changes in frequency over a series of echoes, or "echo train," as opposed to continuous 
frequency contour as one might observe in a signature whistle. The authors also noted that duration cues may be less salient to dolphins listening to echoes in real time, as the differences in duration of the ultrasonic echoes would relatively small.

Helweg and colleagues (1995) investigated the acoustic discrimination of echoes of aspect-dependent objects by humans. In this study, aspect-dependent objects (objects which appear different from different angles, and which produce different echoes when ensonified from different angles) were allowed to move and rotate freely in water while recorded dolphin echolocation clicks were used to create recorded echoes of the objects. Human participants were trained to discriminate among time-stretched versions of series of echoes (echo trains) from the different objects, then were tested on this task. Human performance was compared to that of a dolphin, which had used the same stimuli for the same task. The dolphin effectively demonstrated its ability to discriminate among the objects regardless of the aspect angle of the echoes presented. Human participants were able to discriminate among the echoes from the different objects, but were unable to do so when the echoes were modified to be of equal amplitude. This indicated that humans were reliant upon changes in the amplitude of the echoes across each echo train in order to discriminate among these echoes. This finding is similar to that of DeLong (2017), which examined performance of human listeners discriminating among aspect-dependent objects at a fixed range of novel aspect angles. Human participants found this task difficult, and had to rely upon different auditory cues (e.g., timbre, amplitude, and frequency) than during training at a set aspect angle (DeLong, 2017). Both of these studies show examples of how changing conditions can affect the usefulness of different auditory cues for auditory discrimination in echoic stimuli. 
Another study used the same approach (DeLong et al., 2007a). This study directly compared dolphin and human performance on the same tasks and using the same stimuli. A dolphin was presented with a three-alternative match-to-sample procedure in which he had to discriminate among objects that varied in size, material, shape, and/or surface texture. The dolphin was able to perform above chance for stimuli varying in size, shape, and surface texture, and one set that varied in material, and performed at chance for the other set which varied in material. The next phase of this study was performed using human participants and echoes of the aforementioned stimuli produced using a recorded dolphin click. The echoes were increased in duration by a factor of 125 to ensure that the echoes were played in the human range of best hearing. Participants were first given a list of acoustic vocabulary words with which they might later be able to describe the sounds and their discrimination strategies. Participants were given a training period in which they listened to echoes from all three objects in a given set and could ask to hear an echo any number of times. During the testing phase, participants were presented with an echo train and were instructed to point to the object that they believed the echoes were from, based on their training. After testing, participants were interviewed about the cues used in the discrimination task.

DeLong and colleagues (2007a) found that human listeners performed as well or better than the dolphin and were above chance for all object sets. Most participants expressed using multiple features to discriminate among most stimuli. Though discriminable features differed among the various sets of stimuli, almost all participants cited changes in amplitude, frequency, or timbre across multiple echoes as useful, whereas overall differences in amplitude, frequency, or timbre between objects were less frequently used. This may constitute evidence that the 
changes in auditory features over the duration of a sequence of echoes is a strong cue, particularly when discriminating among objects varying in shape.

DeLong, Au, and Stamper (2007b) performed a study using echoic stimuli from objects that varied in material or wall thickness. Both human listeners and dolphins were able to successfully discriminate among the echoes, and human participants were interviewed about their discrimination strategies. Participants expressed that, for echoes from hollow cylinders made from the same material but with different wall thickness (internal structure), frequency and duration were their most useful cues. When objects were made from different materials, timbre also was useful for echo discrimination (DeLong et al., 2007b). As human and dolphin performance was similar, it is likely that humans and dolphins attended to similar elements of the echoes to achieve their success in the task.

Another study by DeLong and colleagues' (2014) was focused on learning how humans dolphins may recognized aspect-dependent objects (objects varying in shape and material that produce different sounding echoes from different aspect angles). The study instead focused on using echo trains which included echoes from a range of aspect angles, replicating a dolphin's natural head-sweeping motion when using echolocation to observe an object. Participants were trained using echoes from one angle and tested with echoes from novel angles. Participants performed significantly above chance when discriminating among echoes, regardless of whether the angle of the echo was novel or trained. Participants claimed to have utilized the change in amplitude and frequencies across the echo trains when discriminating among the objects. In addition to human listeners, this study employed a neural network to model auditory discrimination of echo trains. The use of neural networks allowed researchers to gain a second source of insight into the values of various cues for echo discrimination, and the researchers 
found that the networks' ratings of the value of different cues closely resembled the value ratings of human participants. These methodologies have granted insight into the mechanisms by which dolphin vocalizations can be interpreted and identified, and similar methodologies may be applied to greater understand the processing of signature whistles.

Branstetter and colleagues (2016b) trained humans and dolphins in a three-alternative match-to-sample paradigm similar to previous studies comparing human and dolphin auditory discrimination. Rather than using echoic stimuli, three physical objects were paired with three sounds designed to resemble dolphin whistles. The baseline versions of these whistle-like sounds were designed to differ from each other in frequency contour, but not in overall frequency, duration, amplitude, or timbre. The baseline whistles were then altered in amplitude, duration, or absolute frequency (leaving the frequency contour cue intact). During the testing phase of the experiment, both human and dolphin participants had to discriminate among the baseline stimuli, as well as the sets of stimuli altered in one of three aforementioned dimensions. Humans were found capable of correctly selecting the target stimulus in every sound transformation condition, which means that they successfully discriminated among the whistles even when they were modified in absolute frequency, duration, or amplitude. Dolphins were able to discriminate among the three targets despite changes in amplitude and duration (though not extreme changes in duration) but did not perform above chance when stimuli were transformed in frequency by a half-octave. This may be indicative of a difference in how dolphins and humans perceive the whistles. Humans may use frequency contour but not absolute frequency to discern meaning. Dolphins, meanwhile, may utilize both and therefore cannot generalize sounds with the same frequency contour but not the same absolute frequency. It is unclear whether this difference in performance is based on inflexible biological factors, such as the brain structures involved in 
sound processing in each species, or based on learned patterns based on prior experience.

However, since this study did not utilize real signature whistles, which vary from each other not only in frequency contour but also in absolute frequency, amplitude, duration, and timbre, it is unclear how either species would have performed with real signature whistle stimuli. It is possible that humans will be able to use overall frequency as well as frequency contour, as dolphins likely do, whenever stimuli differ in these characteristics.

This body of comparative research has generally shown that humans perform at least as well as dolphins at auditory discrimination tasks using echo or whistle-like stimuli (Au \& Martin, 1989; Branstetter et al., 2016b; DeLong et al., 2007a; DeLong et al., 2007b; DeLong et al., 2014). It is likely that human success in the aforementioned tasks will predict success in discriminating between authentic dolphin signature whistles during the present study. What remains to be seen is to what extent masking noise will inhibit this capability.

\section{The Effect of Masking Noise on Human Auditory Perception}

The present study investigates the impacts of specific types of masking noise on human auditory perception and processing of dolphin acoustic signals. Therefore, an understanding of how masking noise affects both humans and dolphins will be crucial to interpret this study's method and outcomes. The study of masking noise and its impact on human auditory perception has primarily focused on perception of human speech and of pure tones masked by simple noise such as pure masking tones and broadband noise (Egan \& Hake, 1950; Green, 1960; Hawkins \& Stevens, 1950; Jon, Lutz, Maria-Liisa, \& Wilhelm, 1999; Marshall \& Jesteadt, 1986; MullerGass, Marcoux, Logan, \& Campbell, 2001; Nelken, Rotman, \& Yosef, 1999). In general, the presence of masking noise does increase the thresholds at which humans will perceive the signal (i.e., the signal must be increased in amplitude to be heard in noise; Egan \& Hake, 1950; 
Hawkins \& Stevens, 1950). The frequency of the masking noise affects the degree to which it will mask a sound, with noise closer in frequency to the stimulus sound having greater impact on the perception of that stimulus, depending on the amplitudes of the signal and the noise (Egan \& Hake, 1950). Egan and Hake (1950) also found that signals with higher frequencies will be masked by a larger band of frequencies.

Evidence has suggested that masking seems to affect perception of both speech and pure tones very similarly, with similar masking thresholds for both types of sounds (Hawkins \& Stevens, 1950). Hawkins and Stevens (1950) found that the difference between the thresholds for speech detection and speech intelligibility was relatively low, ranging from just over $10 \mathrm{~dB}$ at a low level of masking noise $(-56 \mathrm{~dB})$ to about $5 \mathrm{~dB}$ of difference at a high level of masking noise $(+34 \mathrm{~dB})$. In the low-level masking noise, overall threshold to detect speech was about $-55 \mathrm{~dB}$, as opposed to a threshold of over $+15 \mathrm{~dB}$ in a high level of masking noise. The thresholds for perceiving a pure tone in the same amplitudes of masking noise generally fell between the thresholds for detecting and for understanding speech. It is therefore likely that these findings will generalize well to the detection and processing of other types of sounds in noise.

More recent research has determined that human auditory cortices show patterns of processing that are able to process multiple sources of background noise, even when that noise is as complex as background animal sounds such as bird calls (Nelken et al., 1999). Researchers found that sounds were processed by the frequency bands they occupied, and that natural sounds generally were limited to relatively small frequency bands, thus facilitating this process. This research may suggest that, so long as masking noise falls within a relatively small frequency band, humans may be naturally adapted to deal with such noise with relatively little disturbance of perceptual abilities. 
Based on these findings, it seems likely that masking noise will be most impactful on human perception of sounds when the masking noise is high in amplitude (Egan \& Hake, 1950; Hawkins \& Stevens, 1950), similar to the stimulus in frequency (Egan \& Hake, 1950), and covers a broad spectrum of frequencies (Nelken et al., 1999). Some research has also been done on the effects of masking noise and noise pollution on dolphins.

\section{The Effect of Anthropogenic Noise on Dolphins}

Marine anthropogenic noise has a broadly detrimental impact on perception, behavior, and, in extreme circumstances, well-being in all marine animals (Würsig \& Richardson, 2009). In dolphins, which are extremely hearing-reliant, noise has the potential to be particularly impactful. Albuquerque and Souto (2013) found that recreational motorboats have the potential to produce sounds of similar frequency and amplitude to those of dolphin communication, which creates a direct risk of masking at the frequencies dolphins use to communicate. Anthropogenic noise from shipping and other watercraft account for the greatest proportion of potential masking noise within the frequencies of most dolphin signature whistles, as a great deal of these noises occur within a range of frequencies from 1-30 kHz, similarly to signature whistles (Ross, 2005). Furthermore, the amplitudes of motorboat sounds at several sampled frequencies were extremely similar to the amplitudes of those same frequencies during dolphin communication. Thus, this interference would potentially be occurring across dolphins' entire vocal spectra.

A study found that pile driving, in which a machine drives large poles into the ground to provide support for some sort of structure, produces fairly high noise and can have a masking effect over louder vocalizations within a range of 10-15 km, and may mask quieter vocalizations as far away as $40 \mathrm{~km}$ (David, 2006). Though this may be somewhat mitigated by the intermittent nature of pile driving, long-term projects or regions where pile driving is frequent could see 
reduced activity of species affected, and may force groups of dolphins and other species to leave due to difficulties with foraging, communicating, and stress (David, 2006).

A study by Lusseau (2003) on wild bottlenose dolphins in New Zealand demonstrated that behaviors were significantly altered by the presence of tour boats, ranging from groups of one to five kayaks to a single $15 \mathrm{~m}$ catamaran. This altered behavior continued even after the departure of the boats. Rest and social behaviors, which included items such as affiliative contact and group swimming, were the most likely to be disturbed by boat interactions, and bottlenose dolphins most frequently engaged in travel after boat interaction (Lusseau, 2003). This may be indicative of distraction by the physical presence of the boat, interference generated by the boat's noise, or both. The fact that social interactions were particularly impacted may be some indication that social vocalizations were interrupted by boat noise, though the study did not measure dolphin vocalizations in order to support or reject this possibility.

Another study observed acoustic information and dolphin behavior during boat-dolphin encounters and found that the interruption of foraging behavior was correlated with boat presence, but not with boat noise (Pirotta et al., 2015). This may indicate that the presence of the boat is more imposing or distracting than its noise. It is unclear if this might indicate some degree of resilience of dolphin vocalizations to masking. Further research, ideally using samples of boat noise in a controlled experimental setting, ought to expand upon this phenomenon and determine the role that masking may play in these interactions.

Kaplan and Mooney (2015) conducted an acoustic survey of the U.S. Virgin Islands National Park and found boat noise in approximately $6 \%-12 \%$ of their samples. This noise included frequencies which could potentially mask local marine life, and contributed significantly to the total amount of environmental noise when boat noise was present. It was 
suggested that this amount of boat traffic, combined with its amplitude and frequencies, might impair local species' (including species of dolphins) abilities to communicate and perceive their environments (Kaplan \& Mooney, 2015). For species such as dolphins, this could result in impaired foraging ability and potentially force pods of dolphins to seek out locations with less boat traffic, either restricting them to specific parts of the park, or forcing them to leave the park entirely. This is likely to be a chronic problem for dolphins worldwide, due to the extreme distances sound is able to travel through seawater. Sounds at mid-range frequencies $(500 \mathrm{~Hz}$ to $50 \mathrm{kHz}$ ) can travel for multiple kilometers before attenuating, and low-range frequency sounds (10 Hz to $500 \mathrm{~Hz}$ ) can travel hundreds of kilometers (Hildebrand, 2009). These low and midrange frequencies are also the ranges at which most marine vessels generate the most noise (Hildebrand, 2009). Given the abundance of boats in most of the world's oceans, combined with the gradual attenuation of sound in the marine environment, it is likely that boat noise is extremely common for most dolphins, especially those living in coastal waters. Busy ports, especially those which harbor large commercial vessels, may produce constant noise at frequencies which could impact an extremely wide radius.

The extent to which masking noise might impair a dolphin's ability to echolocate has been studied in great detail with animals in human care (e.g., Au et al., 1988; Au \& Penner, 1981; Branstetter et al., 2016a; Branstetter, Trickey, Aihara, Finneran, \& Liberman, 2013a), revealing thresholds at which sounds affect dolphins' ability to detect or discriminate among objects using echolocation. In general, this research suggests that, like in humans, the effects of masking noise vary with both the frequency and amplitude of both the noise and the signal. Unsurprisingly, masking noise can impair dolphins' abilities to echolocate accurately, which could impact their ability to forage. One study (Lemonds, Au, Vlachos, \& Nachtigal, 2012) 
which examined a bottlenose dolphin's masked hearing thresholds of a pure tone, found similar masking patterns to the aforementioned body of research (Au et al., 1988; Au \& Penner, 1981; Branstetter et al., 2016a; Branstetter et al., 2013a) on masking and echolocation. Branstetter and colleagues (2016a) studied the effects of several types of natural and artificial noise sources on dolphin detection of various natural and artificial dolphin vocalizations: one non-signature whistle, one burst pulse, echolocation clicks, a $10 \mathrm{kHz}$ tone, and four artificial, whistle-like sounds. The experiment used natural samples of noise from ice movement, a major source of noise in northern oceans, and snapping shrimp, a major source in tropical and some subtropical oceans. In addition, the study used two types of artificially-generated noise. It is crucial to note that some signals, especially echolocation clicks, were masked more or less severely than others by certain noises (Branstetter et al., 2016a). This disparity in masking patterns across different noise types emphasizes the need for further study of specific signal types and noise types, especially commonly-used signals such as signature whistles. Masking threshold research has not been performed using signature whistles, but it is likely that masking noise would similarly impair dolphins' abilities to perceive these signals. Given the importance of signature whistles alone for social cohesion, bonding, and between-pod interactions, there could be potentially dire effects if masking impairs the functionality of this element of communication.

It has been found that wild dolphins engage in significantly more use of signature whistles during the approach of marine vessels (Buckstaff, 2004). Buckstaff (2004) recorded vessels ranging from 3 to $30 \mathrm{~m}$ in length and of varied propulsion types, ranging from kayaks to powerboats. This is likely an indication that boats do mask whistles and conspecific communication as a whole, thus necessitating increased repetition. An alternative interpretation might be that the frequent and repeated use of a dolphin's signature whistle may indicate 
agitation or stress, which has been suggested in previous work (Janik \& Sayigh, 2013; Janik \& Slater, 1998). The study by Buckstaff (2004) found significant differences between the noise levels of boats depending on their state of travel. Different speeds result in these different states, with planing boats, which move the fastest, resulting in the front of the boat lifting out of the water, being the loudest, plowing boats, which move slower and with the hull parallel to the water, being second loudest, and with idling being the quietest. The study also determined the volume of noise that the dolphins were exposed to by such watercraft, which may allow for the creation of more realistic masking conditions. The loudest recorded noise was $26 \mathrm{~dB}$, from a planing boat which passed within about $25 \mathrm{~m}$ of the recording equipment. On average, planing boats produced noise about $18 \mathrm{~dB}$ above that of the natural environment (Buckstaff, 2004).

Due to the fact that many populations of wild dolphins frequent areas that are exposed to anthropogenic noise with the potential to mask their communication (Albuquerque \& Souto, 2013; David, 2006; Kaplan \& Mooney, 2015), it is important to understand how such noise might affect their ability to communicate. Thus, this study will investigate how masking noise might affect auditory discriminatory abilities with respect to dolphin communicative vocalizations, specifically signature whistles.

\section{Current Study}

The current study utilized human listeners to gain insight into auditory discrimination in dolphins. Though there are many extant species of dolphins, this research focused on the Atlantic bottlenose dolphin, a widely-ranging species with well-studied perceptual and cognitive abilities (e.g., Au, 1993; Mercado \& DeLong, 2010). Humans have served as a model organism in several studies in order to model perceptual processes in dolphins (e.g., Au \& Martin, 1989; Branstetter et al., 2016b; DeLong, 2017; DeLong et al., 2007a; DeLong et al., 2007b; DeLong et al., 2014; 
Gorman \& Sawatari, 1985). This framework allows researchers to inquire as to the strategies that human participants use in order to complete the task, such as which features of a sound the participants attend to when discriminating between stimuli. When human participants' results are compared to those of dolphins performing the same task, and then the human participants' declared strategies are considered, one can begin to make inferences about which strategies dolphins may also be employing. Currently, this type of research has examined echo perception and discrimination strategies (DeLong, 2017; DeLong et al., 2007a; DeLong et al., 2007b; DeLong et al., 2014). One study has also examined human strategies in discriminating whistlelike sounds with many similar features to dolphin whistles, but not real dolphin signature whistles (Branstetter et al., 2016b).

The current study was the first to investigate human auditory perception of authentic dolphin signature whistles. The study also endeavored to determine to what extent anthropogenic boat noise of the type that is likely to be encountered by dolphins may impede human auditory discrimination of dolphin signature whistles. One advantage of the current study was the use of sample boat noises from an area where dolphins are relatively common: the U.S. Virgin Islands. The current study attempted to determine how the discrimination strategies of the human listeners changed with and without masking. First, participants were trained and tested for discrimination among baseline whistles, which lacked any masking boat noise. Then participants were tested with signature whistles masked by five different samples of anthropogenic boat noise. The signal to noise ratio were varied to estimate the threshold at which human listeners' ability to discriminate among signature whistles decreased.

I hypothesized that humans would be able to auditorily discriminate between authentic signature whistles at baseline, without background noise, since humans have previously been 
found successful in discriminating among sounds meant to imitate dolphin whistles (Branstetter et al., 2016b). I hypothesized that the ratio of signature whistle amplitude to boat noise amplitude will affect discrimination accuracy by human listeners, as higher amplitude masking noise generally increases human thresholds of detection and processing of sounds (Egan \& Hake, 1950; Hawkins \& Stevens, 1950). Specifically, I hypothesized that low signal to noise ratio would result in poorer performance. I also hypothesized that boat noise with frequencies similar to the signature whistles will create the most problems for discrimination, as previous work suggests that the frequency of masking noise plays a role in its impact on hearing thresholds (Egan \& Hake, 1950; Nelken et al., 1999). Finally, if the previous hypotheses are confirmed and noise impacts the difficulty of the task, I predicted that participants would alter their listening strategies, such as placing greater emphasis on different auditory cues in trials where noise is present.

\section{Experiment 1}

The first experiment investigated to what extent human participants were able to auditorily discriminate among baseline signature whistles. Participants listened to and identified signature whistles from dolphins $\mathrm{A}, \mathrm{B}$, and $\mathrm{C}$ without boat noise.

\section{Method}

Participants. Sixteen participants (nine male and seven female) participated in this study. Participants ranged in age from 18 to 23 years in age $(M=20$ years). There were 2 participants in the pilot study and 14 participants in Experiment 1. Only individuals with normal hearing were able to participate, as this study involved discriminating among auditory stimuli. Participants were screened using a standardized hearing test (Home Audiometer, 2017) to ensure adequate hearing ability at 125, 250, 500, 750, 1,000, 1,500, 2,000, 3,000, 4,000, 6,000, and 
$8,000 \mathrm{~Hz}$ in both ears. All participants successfully passed this test. Participants were asked questions about their musical experience, musical ability, typical exposure to noise, and family history of hearing loss (Appendix A). Of the 16 participants, three had previously participated in hearing studies, one reported having had a previous hearing problem (preferred not to give further information), none reported having any current hearing-related difficulties, and none reported a family history of hearing loss. Eleven participants had musical experience, among whom the average self-reported ability level was 3.25 on a Likert scale from 1 to 7 . Ten participants reported working in a noisy environment, with an average rating of 3.67 for the prevalence of noise in these scenarios on a Likert scale from 1 to 7 . Of the participants who reported working in a noisy environment, only one reported using hearing protection during work. Participants rated their frequency of music-listening to be an average of 5.67 on a Likert scale from 1 to 7 , most commonly using in-ear headphones and a volume of 4.4 on a Likert scale from 1 to 7. These data on hearing issues, musical experience, and noise exposure were collected to help describe this sample of listeners, but were not analyzed with the performance data. Participants were recruited using the Rochester Institute of Technology SONA system and paper flyers, and were compensated for their time with course credit (6 SONA credits) or $\$ 10$.

Materials. This study utilized Audacity® 2.22, 2018 for the editing and assembly of auditory stimuli. Participants used Bose ${ }^{\circledR}$ On-Ear headphones (Bose Corporation, 2006) to listen to stimuli. Qualtrics ${ }^{\circledR}$ was used as the software platform for presenting the training and test phases on a 14 inch IdeaPad Y700 laptop computer (Lenovo Group Ltd., 2015). Sound pressure levels were standardized using Audacity® 2.22, 2018 and checked using a digital sound level meter (Model SLM01, Tacklife). 
The auditory stimuli consisted of signature whistles from three individual adult male dolphins, which were referred to throughout the study as "Dolphin A," "Dolphin B," and "Dolphin C." All three dolphins resided in the same facility in Florida when the whistles recordings were made. Dolphins A and B shared a father. Dolphin A was 14 years of age, Dolphin B was 17 years old, and Dolphin C was 27 years old at the time their signature whistles were recorded. There were six exemplars of signature whistles from each dolphin (one for training, five for testing), for a total of 18 signature whistle files (see Figures 1-3 for examples from each dolphin). Signature whistles were recorded in the facility in Florida using a hydrophone array with a sampling rate of at least $62.5 \mathrm{kHz}$. Signature whistles were primarily determined by recording isolated dolphins and determining the most common whistle. Some whistles were not recorded in isolation, but could be identified as matching a dolphin's signature whistle by the whistle's frequency contour. Whistles were identified and matched through visual analysis of spectrograms. The identity of the dolphin producing each vocalization was collected by having trainers note the proximity of each dolphin to the hydrophones positioned around the dolphins' enclosure.

An entry survey was used to collect demographic information about participants, as well as information about their hearing status, musical experience, experience with listening in noise, and exposure to noise (Appendix A). Participants were trained by an experimenter, who read from a paper script during non-computerized parts of each session, and from a script on the same computer that was used to play training samples and trials for participants during the listening task (see Appendix B for this training script). As part of their training, participants were given a printed handout that explained several terms relevant to hearing and auditory perception: amplitude, duration, frequency, frequency contour, timbre, and other cues (Appendix C). 
Interviews given to the participant were read from a printed form by the experimenter (Appendix D).

Procedure. The procedure included a pilot study followed by the experimental test sessions.

Pilot study. The pilot study for Experiment 1 used the same methods and followed the same procedure as the experimental sessions. The pilot study also helped to ensure that the training methodologies were sufficient and that participants' interpretations of the training and listening task instructions were consistent.

Two participants volunteered for the Experiment 1 pilot study. Participants achieved an average accuracy of $99.58 \%$ during the listening task, which helped to estimate the necessary sample size for Experiment 1 by using a power analysis. Based on the results of this power analysis, it was determined that in order to support the hypothesis that humans could successfully discriminate among three signature whistles, Experiment 1 would have required one to two participants. I recruited more than two participants for added certainty and to increase the power level for analysis of participants' responses to the interview, and to test approximately the same number of participants that have been used in similar studies (e.g., Au \& Martin, 1989; Branstetter et al., 2016b; DeLong, 2017). Additionally, pilot study participants' interview responses were checked to ensure that responses addressed the intended questions, and that participant interpretations of all items were consistent. Overall, participants were fairly consistent in their interview responses, and no problematic questions were encountered. As a result, the questionnaire was not altered after the completion of the pilot study.

The pilot study ensured that the experiment took the intended period of time. Exceeding the intended period of time would have impacted the appropriate compensation for participants, 
and might have led to concerns of participant fatigue during the experimental procedure, potentially leading to errors not representative of the difficulty of the individual stimuli or the task. The task typically took 50 to 60 minutes to complete in the pilot study, which was deemed appropriate for the conditions of this study.

Experimental test sessions. A single experimenter tested participants in a sound-treated chamber (Controlled Acoustical Environments, Industrial Acoustics Company, Inc., Bronx, NY) on the Rochester Institute of Technology campus. For the duration of the experiment, the experimenter sat opposite the participant, and the computer faced towards the experimenter and away from the participant. Participants first took a brief (approximately 10 minute) hearing test in order to ensure they had no signs of hearing loss which might impair their ability to perform the study's listening task. All participants met the standards for normal hearing. After passing the listening task, participants filled out the entry survey (Appendix A). Participants then were trained for the listening task, tested on their signature whistle discrimination abilities, and interviewed about their listening and discrimination strategies. The experimenter remained present throughout the experiment. It took participants approximately 60 minutes total to complete the entire procedure.

Training phase. Participants were given verbal instructions for the task. The experimenter followed prompts on the computer screen to ensure consistent participant experience. Participants were given a brief explanation of signature whistles (see Appendix B) and were presented with a list of vocabulary to consider while performing all training and testing phases of the study, defining the terms "amplitude," "frequency," "frequency contour," "timbre," and “duration," (see Appendix C). Participants also received a printed copy of this vocabulary list, which they were told to reference at any time during the experiment including during test phases 
and interviews. The experimenter played sample sounds over the computer's speakers which further demonstrated each of these properties of a sound from the vocabulary sheet, similar to previous human audition studies which include an interview phase (e.g., DeLong et al., 2007a). The experimenter encouraged participants to also attend to cues that are not necessarily on their vocabulary sheet and expressed that participants were encouraged to discuss any such additional cues during the interviews.

The experimenter described the test phases of the experiment, and then played one sample whistle from "Dolphin A," "Dolphin B," and "Dolphin C." These samples were a sixth whistle from each dolphin, not used during the listening task itself (Exemplar 0 for each dolphin). Each of these sample whistles was played three times for every participant, and the participants were encouraged to ask the experimenter to play any of the whistles additional times until the participant felt confident in their ability to discriminate among the stimuli. The participants were encouraged to note specific elements of each whistle, such as the acoustic features on their vocabulary sheet, that might allow them to more easily discriminate among the whistles later on. The training phase typically took about five minutes.

Testing phase. For the test phase, the experimenter would play the stimulus, record the participant's response, inform the participant whether they were correct or wrong, then immediately begin the next trial. The experimenter played each whistle only once per trial. Participants were presented with all five baseline exemplar whistles for each of the three dolphins, with each whistle occurring 16 times during the test phase. In total, participants underwent 240 trials in the test phase of Experiment 1 . There was a total of 16 consecutive blocks, each made up of 15 trials with one sample of each exemplar from each dolphin, and the order of trials within each block of 15 were randomized. After completing 120 of the 240 trials, 
participants were given a two-minute break during which they were asked to take off their headphones, in order to avoid possible effects of fatigue. The testing phase of Experiment 1 typically took about 45 minutes.

Interview. After the completion of the test phase, the experimenter administered a brief verbal interview. First, the experimenter played one exemplar whistle from each dolphin, the same exemplar as was used during the training session. The experimenter then led a structured interview in which the participant answered questions about the extent to which they utilized various acoustic features of the whistles in order to identify signature whistles. The interview questions and recording form is included in Appendix D. For Experiment 1, the experimenter only asked questions $1-4$. The interview typically took about 5 minutes.

\section{Results}

Performance accuracy. Participants in Experiment 1 achieved near-perfect performance on the signature whistle discrimination task $(M=98.94 \%, S D=1.57 \%)$. For this task, chance performance would be approximately $33.33 \%$. A Student's t-test was used to compare actual and chance performance, in order to test the hypothesis that participants would be able to discriminate auditorily among dolphin whistles. Participant performance was significantly better than chance, $t(13)=156.21, p<.001$. Although there were no predictions concerning significant differences in performance between whistles of the three dolphins or among the 16 test blocks, exploratory analyses showed similar mean performance accuracies for the three dolphins $(M=$ 99.73\%, 95\% CI: [98.60\%, 100.87\%] for Dolphin A, $M=98.75 \%, 95 \%$ CI: [96.31\%, 101.19\%] for Dolphin B, and $M=98.04 \%, 95 \%$ CI: [94.99\%, 101.08\%] for Dolphin C) and some differences in performance on some of the blocks, particularly the first block (Table 1). 
Interview responses. All participants reported hearing a difference between the three dolphins' whistles. Of the 14 participants, all 14 reported a difference in frequency contour, 13 reported a difference in timbre, 12 reported a difference in duration, 11 reported a difference in frequency, 7 reported a difference in amplitude, and 4 reported another difference. One reported "other" difference, for example, was the perceived harshness of the whistle, expressing a degree of emotional valence to at least one of the whistles. The majority of participants in Experiment 1 (12 of 14) reported the whistles of Dolphins B and C as being the most similar and easily confused. Participants were most likely to report frequency contour either as the primary factor in this confusion or one of two main factors (11 of 14 participants). Participants' ratings of how frequently they used each auditory cue during the listening tasks during Experiments 1 are given in Figure 4. Participants generally claimed to use frequency contour the most frequently. Participants' assessments of which cue or cues were most helpful for identifying each individual dolphin during Experiment 1 are given in Table 2. Frequency contour and duration were the most frequently given cues, but participants reported using cues differently for different dolphins. Frequency contour was reported by the majority of participants for all three dolphins, whereas duration was reported by 9 of 14 participants for dolphin A and only 2-3 participants for dolphins B and C. Timbre was reported for 7 of 14 participants for dolphin $\mathrm{C}$ but $0-1$ participants for dolphins $\mathrm{A}$ and $\mathrm{B}$.

\section{Discussion}

One of the primary objectives of Experiment 1 was to test how well human participants could auditorily discriminate among the signature whistles of the three dolphins. In this experiment, there was no masking noise present, and whistle amplitude was high (approximately $55 \mathrm{~dB}$ ). As predicted, participants were able to discriminate among the signature whistles. In 
fact, participants under these conditions exhibited near-perfect performance on the signature whistle discrimination task, achieving an average accuracy of almost 99\%. Participant performance was so high that it is possible any errors that did occur among participants were the result of random chance, for example, intending to report one whistle and stating a different one by accident due to inattentiveness. Exploratory analyses indicated that participant performance may improve or become more consistent over the course of the earliest blocks of the experiment. Participants excelled at this task immediately, and improved extremely quickly. The speed with which human listeners became proficient with auditory whistle identification may serve to further validate the use of humans as a model listener for this type of study.

Participants' responses in the interview seem to indicate that, in this experiment, frequency contour was consistently the most helpful cue. This is consistent with Branstetter and colleagues' (2016b) study on human and dolphin perception of whistle-like sounds, which showed that humans are capable of discriminating among stimuli with near-perfect accuracy even when frequency contour is the only cue available by which to make this discrimination. Evidence from studies using artificial neural networks have indicated that frequency contour is key for identifying signature whistles (e.g., Janik, 1999). Humans also use frequency contour (the change in frequency across a series of echoes instead of a continuous sound) when discriminating among dolphin biosonar echo trains, especially to identify the shape of objects (e.g., DeLong et al., 2014). Finally, humans have previously been shown to be able to discriminate among signature whistles visually, using spectrograms, even when frequency contour is the only cue visualized in the spectrograms (e.g., Sayigh et al., 2007). Therefore, it is unsurprising to find that participants in Experiment 1 cited frequency contour as being the cue that they used most frequently to help identify the dolphin whistles during the task. 
The interview asked participants to quantify the usefulness of each auditory cue for identifying the signature whistles, yielding a more in-depth breakdown of how much each cue factored into participants' identifications of the signature whistles than in prior studies (Au \& Martin, 1989; Branstetter et al., 2016b; DeLong, 2017; DeLong et al., 2007a; DeLong et al., 2007b; DeLong et al., 2014; Gorman \& Sawatari, 1985). For example, participants were able to rate the frequency of use for each cue and also specifically state which cue was their most-used cue. Exploratory analyses indicated that participants also reportedly used different cues to identify the whistles of different dolphins (Table 2). The greatest examples of this were use of duration and timbre as acoustic cues. Nine participants reported using duration to identify Dolphin A's whistle, which was particularly short, while only two and three participants reported using duration for Dolphin B and C, respectively. Likewise, no participants used timbre for Dolphin A, and only one participant reported using timbre for Dolphin B, but seven participants reported using timbre for Dolphin C. This might indicate the presence of some variability in listening strategy depending on the circumstances of the task. When this experiment is compared to dolphins' overall performance and patterns of errors while attempting the same task in future research, it might grant insights into what strategies dolphins use and how these strategies may differ from those of human listeners, as in DeLong and colleagues' research (2007a).

\section{Experiment 2}

The second experiment investigated to how the presence of boat noise affected participants' ability to auditorily discriminate among signature whistles. Participants discriminated among signature whistles from dolphins A, B, and C, with different ratios of signal to boat noise. 


\section{Method}

Participants. Forty-four participants (22 male, 21 female, 1 genderqueer) participated in this study. Participants ranged from 18 to 43 years in age $(M=21$ years). Pilot Study A, Pilot Study B, and Experiment 2 had 2, 6, and 36 participants, respectively. Participants were recruited from the same population as those in Experiment 1 using the same recruitment techniques. Participants underwent the same screening procedure, and received the same compensation. All participants passed the hearing test and were allowed to participate. Of the 44 participants, two had previously participated in hearing studies, one reported having had a previous hearing problem (eardrum reconstruction surgery due to frequent ear infections during childhood), and one reported having difficulty hearing a specific type of sound (quiet, low-frequency voices). These participants were not removed because they passed the hearing test. Thirty-one participants had musical experience, among whom the average self-reported ability level was 3.56 on a Likert scale from 1 to 7 . Sixteen participants reported working in a noisy environment, with an average rating of 3.16 for the prevalence of noise in these scenarios on a Likert scale from 1 to 7. Of the participants who reported working in a noisy environment, three reported using hearing protection during work. Participants rated their frequency of music-listening to be an average of 5.93 on a Likert scale from 1 to 7 , most commonly using in-ear headphones and a volume of 4.3 on a Likert scale from 1 to 7 . These data on hearing issues, musical experience, and noise exposure were collected to help describe this sample of listeners, but were not analyzed with the performance data. Participants were compensated for their time with course credit (6 SONA credits) or $\$ 10$.

Materials. This experiment used the same materials as Experiment 1, with the exception of the auditory stimuli used during the test phase. This study utilized noise samples to mask three 
quarters of the auditory stimuli. Boat noise samples were recorded by Kaplan and Mooney (2015) in the U.S. Virgin Islands National Park. Sounds were collected from three reefs and were sampled for about one minute every two hours, providing samples from a variety of times of day. Recordings were made using autonomous underwater recording devices with hydrophones positioned approximately $0.3 \mathrm{~m}$ above the sea floor. Boat noises were then detected within sample recordings using visual identification and auditory confirmation. A subset of 28 of these recordings containing boat noise, collected between May 30, 2016 and March 29, 2017, were considered for use in this study. The six samples with the highest peak amplitudes of boat noise lasting more than two seconds (long enough to fully mask all signature whistle exemplars) were selected for use in Experiment 2 (Table 3). See Figure 5 for spectrograms of the boat noise samples.

Whistle stimuli were presented both with and without masking noise from boats for Pilot Studies A and B, as well as the experimental sessions. Audio files were created which contained simultaneous samples of boat noise and dolphin whistles. Each of the six sample boat noises were paired randomly with one exemplar from each dolphin using a random number generator (Randomness and Integrity Services Ltd.). The amplitudes of the individual tracks containing either boat noise or signature whistles were adjusted to create different ratios of signal to noise, based on the maximum amplitude of each sample. There were two levels of amplitude for the signature whistles, low amplitude and medium amplitude. There were four levels of amplitude for the noise samples, no amplitude, low amplitude, medium amplitude, and high amplitude. See Table 4 for the complete list of combinations of signal and noise amplitudes for the signal to noise condition. See Figure 6 for an example of a signature whistle masked by boat noise. 
Procedure. The procedure included pilot studies followed by the experimental test sessions. Both the pilot test and the experimental sessions used the same procedure, with the same protocol for training, testing, and the follow-up interview.

Pilot Study. Two pilot studies were performed prior to the experimental test sessions, which used the same methods and followed the same procedure as the experimental sessions. The goal was to determine a set of signal to noise conditions where at least the most difficult condition would force participants to perform at or below chance. In Pilot Study A, signal to noise ratios ranged from about $67.5 \mathrm{~dB}$ of signal with no amplitude of boat noise at the easiest to about $62.5 \mathrm{~dB}$ of signal with about $67.5 \mathrm{~dB}$ of boat noise at the hardest. Participants still performed exceedingly well $(M=97.71 \%)$, indicating that the conditions weren't difficult enough to induce error. In Pilot Study B, both boat noise and signal amplitudes were changed to create larger differences between the different conditions. The new signal to noise ratios ranged from about $70 \mathrm{~dB}$ of signal versus no amplitude of boat noise at easiest to about $60 \mathrm{~dB}$ of signal versus about $70 \mathrm{~dB}$ of boat noise at hardest. Participant performance decreased $(M=90.97 \%)$, but not dramatically. Performance ranged from an average of $96.67 \%$ in the easiest condition to $69.44 \%$ in the hardest condition, indicating that participants would still perform well above chance in all conditions. Based on these findings, the stimuli for the main experiment were altered by decreasing signature whistle amplitudes for half of the trials. The resulting signal to noise ratios can be seen in Table 4.

Experimental sessions. The experimental sessions included a training phase, test phase, and interview phase, similar to Experiment 1. Experiment 2 used the same procedure as Experiment 1, with two exceptions: some of the stimuli contained boat noise as well as signature whistles at different ratios of signal to noise. In addition, the interview was changed slightly; the 
first half of the interview contained the same questions as Experiment 1, asked specifically with regards to the trials in which noise was absent. Following this, participants were asked the same questions about the trials when noise was present (Appendix E). Experiment 2 typically took a total of about an hour and 15 minutes to complete.

Training Phase. The training procedure was exactly the same as in Experiment 1, except that participants were informed that some trials would contain other sounds as well as a signature whistle, and that every trial would contain one signature whistle for them to identify.

Testing phase. The testing phase was the same as in Experiment 1, with a few exceptions. Instead of listening only to baseline signature whistles (without noise), participants were also asked to identify signature whistles masked by boat noise of various amplitudes. In half of the trials, the amplitude of the signature whistle was also reduced. There was a total of 80 trials that included whistles from each of the three dolphins, for a total 240 trials. Each session was divided into two blocks. Each block contained one trial for each whistle exemplar-boat noise pairing at eight levels of signal and noise, for a total of 120 trials per block. Each participant received the same trials in both block 1 and block 2 , but the order in which the trials were presented was randomized separately for each participant. The listening task took approximately 30 minutes to complete. The experimenter administered a brief verbal interview immediately after the completion of this task.

Interview. At the beginning of the interview, the experimenter informed the participant that the first part of the interview was solely with regard to the trials where there was no background noise present. The experimenter then played the training exemplars from the beginning of the experiment, and informed the participant that they could ask for the whistles to be repeated at any time during the interview if they wanted to reference them. This was intended 
to get the participants focused on and considering the trials during which there was no boat noise present. The experimenter then led a structured interview in which the participant answered questions about the extent to which they utilized various acoustic features of the whistles to discriminate successfully during the trials where noise was not present. The experimenter then informed the participant that they were moving on to the part of the interview which referred to the trials where noise was present. The experimenter played samples of signature whistles masked with boat noise, with one example for each dolphin not used during the listening task (exemplar 0 for each dolphin and noise 0, Figures 1-3 and Figure 5, respectively). Participants were then asked similar questions about their listening strategies in the presence of noise. The interview questions and recording form are included in Appendices D and E. The interview typically took about 5 minutes.

Data analyses. Multilevel logistic regression was used to investigate how participant performance was affected by the boat noise sample, signal to noise ratio, and similarity of frequency between signature whistle and boat noise sample on participant performance using RStudio 1.1.463 (RStudio, Inc., 2018). Multilevel logistic regression was selected because it can be used to analyze binomial data with low variability, and because it allowed for a mixture of categorical and continuous independent variables. This strategy was used to address the hypothesis that signal to noise ratio would affect performance, and the hypothesis that similarity between the frequencies of the boat noise samples and the signature whistles would affect performance. The first model assessed the impact of different boat noise samples as a categorical variable, the impact of signal to noise ratio, and the interaction of these effects on participant performance as a repeated binomial measure. The model also included possible main effects of test block and dolphin, as well as possible two-way interaction effects between test block and 
dolphin, test block and boat noise sample, test block and signal to noise ratio, and dolphin and signal to noise ratio, with all of the listed effects treated as covariates and not included in hypothesis testing. Test block was included as a covariate to control for the possible effects of practice or fatigue, and dolphin was included in case the signature whistles from any of the dolphins might be more or less identifiable.

In the event that boat noise sample had a significant effect on performance, a follow-up model was planned to investigate whether this effect could be attributed to the frequency of the boat noise samples. The second model tested for two interaction effects: the interaction between boat noise peak frequency and signal to noise ratio and the interaction between boat noise peak frequency and dolphin peak frequency. Boat noise peak frequency would only be tested as a main effect if the previous model did not find a significant interaction between boat noise sample and signal to noise ratio. As covariates, not included in hypothesis testing, the model included main effects of test block, boat noise peak frequency, whistle peak frequency, and signal to noise ratio, as well as the interaction effects between test block and dolphin whistle peak frequency, test block and boat noise peak frequency, test block and signal to noise ratio, and dolphin whistle peak frequency and signal to noise ratio. Test block was again included as a covariate to control for the possible effects of practice or fatigue. Signal to noise ratio was included to control for any main effect which might be shown in the first model. Boat noise peak frequency was included in case frequency accounted for any possible effect which might be shown in the first model. Signature whistle peak frequency was included in case the frequency accounted for the possibility that any of the dolphins might be more or less identifiable. 


\section{Results}

Performance accuracy. Participants in Experiment 2 performed well overall $(M=$ $80.68 \%, S D=6.36 \%$ ). To investigate the hypotheses that both the signal to noise ratio and the similarity of the signal and boat noise would all affect performance, a 5 (boat noise sample) $\times 8$ (ratio of signal to noise) multilevel logistic regression model (Raundenbush \& Bryk, 2002) was used, controlling for test block and dolphin (A, B, C), with an experimental type I error level of $\alpha$ $=0.05$ and Holm's correction (1979) for multiple hypothesis testing. This model also tested a possible two-way interaction between boat noise sample and signal to noise ratio. The model controlled for possible two-way interactions between block and dolphin, block and boat noise sample, block and signal to noise ratio, and dolphin and signal to noise ratio. There was no significant main effect for boat noise sample $\left(\chi^{2}(4)=7.05, p>.05\right)$.

There was a significant main effect for signal to noise ratio $\left(\chi^{2}(7)=135.52, p<.01\right)$. Post-hoc analyses were conducted to further investigate this main effect, using Tukey's (1949) HSD to correct for multiple comparisons. The means, 95\% confidence intervals, and groupings based on statistically significant differences are included in Table 5 . The odds ratios and results from each pairwise comparison are included in Table 6. Participant performances at different ratios of signal to noise are depicted in Figure 7. Conditions could be grouped into approximately three levels of performance, all significantly different: low or no impairment, moderate impairment, and severe impairment. In the low signal amplitude trials, performance was moderately impaired by medium noise and severely impaired by high noise. In the medium signal amplitude trials, performance was moderately impaired by high noise.

There was a significant interaction effect between boat noise sample and signal to noise ratio $\left(\chi^{2}(28)=95.51, p<.001\right)$. Post-hoc analyses of the interaction effect were conducted using 
Holm's correction (1979) for multiple comparisons. Boat noises 1-5 were compared within each level of signal to noise ratio. The means, $95 \%$ confidence intervals, and results of the post-hoc analyses are included in Table 7. Participant performances for different boat noise samples at different ratios of signal to noise are displayed in Figure 8. In three signal to noise ratio conditions (low signal with no noise, medium signal with no noise, and medium signal with low noise), there were no differences between the five boat noises. In five signal to noise ratio conditions, participants performed significantly better on some boat noises than others, usually performing best on trials with boat noise 1 , and worst on trials with boat noise 3 . This was especially true in the conditions with moderate to severe performance impairment due to noise.

The significant interaction effect found for boat noise sample and signal to noise ratio indicated that some acoustic property of the boat noise samples may affect performance. To analyze the hypothesis that proximity in frequency between dolphin signature whistles and boat noise samples could impair performance, a second multilevel logistic regression model (Raundenbush \& Bryk, 2002) was used. In this model, the peak frequencies of each boat noise sample and of each dolphin's whistle were used as continuous variables instead of simply using dolphin and boat noise sample as categorical variables. This multilevel logistic regression model (Raundenbush \& Bryk, 2002) controlled for test block, with an experimental type I error level of $\alpha=0.05$ and Holm's correction (1979) for multiple hypothesis testing. Because the previous model showed an interaction effect where boat noise sample seemed to only affect performance at certain ratios of signal to noise, this model tested for a possible two-way interaction between boat noise peak frequency and signal to noise ratio. The model also tested for a two-way interaction between boat noise peak frequency and dolphin whistle peak frequency, to determine whether similarity of frequency between the whistle and boat noise played a role, or if 
performance was affected only by the frequency of the boat noise. The model controlled for possible interactions between block and dolphin whistle frequency, block and boat noise frequency, block and signal to noise ratio, and dolphin whistle frequency and signal to noise ratio. The model could not test for three-way interactions due to the relatively low variance within smaller subgroups of data.

There was a significant interaction effect between signal to noise ratio and boat noise peak frequency $\left(\chi^{2}(7)=28.12, p<.001\right)$. Post-hoc analyses of the interaction effect were conducted using Holm's correction (1979) for multiple comparisons. The peak frequencies of boat noises 1-5 were compared within each level of signal to noise ratio. The means, $95 \%$ confidence intervals, and results of the post-hoc analyses are included in Table 8 . There were no significant differences in performance based on boat noise frequency at higher ratio of signal to noise conditions: low signal and no noise, low signal and low noise, medium signal and no noise, and medium signal and low noise conditions (all $p^{\prime} \mathrm{s}>.05$ ). In conditions with low signal and medium noise, low signal and high noise, medium signal and medium noise, and medium signal and high noise, there was found to be a significant relationship between performance and the peak frequency of the boat noise sample. In each of these conditions, performance was worst with the boat noise with the highest peak frequency (all $p^{\prime} \mathrm{s}<.05$ ).

There was also a significant interaction effect between boat noise peak frequency and dolphin whistle peak frequency $\left(\chi^{2}(1)=10.45, b=1.55, \mathrm{SE}=0.48, p<.001\right)$. Post-hoc analyses of the interaction effect were conducted using Holm's correction (1979) for multiple comparisons. The peak frequencies of dolphins A, B, and C were compared within each boat noise peak frequency condition. The means, $95 \%$ confidence intervals, and results of the posthoc analyses are included in Table 9. In the presence of the lowest-frequency boat noise samples 
(Noise 1 at $0.063 \mathrm{kHz}$ and Noise 4 at $0.132 \mathrm{kHz}$ ), there was no relationship found between noise frequency and whistle frequency (all $p$ 's $>.05$ ). In the presence of all three of the higherfrequency boat noise samples, performance was significantly worse in conditions with lower whistle frequency (all $p$ 's $<.05$ ), indicating that more similar frequencies between boat noise samples and dolphin whistles did cause worse performance (i.e., performance was worst on Dolphin C, whose whistle had the lowest peak frequency, and was closest to the lower peak frequencies of the boat noises).

Although there were no predictions concerning significant differences in performance between whistles of the three dolphins or between the two test blocks, exploratory analyses showed different mean performance accuracies for the three dolphins $(M=98.1 \%, 95 \% \mathrm{CI}$ : [97.1\%, 98.8\%] for Dolphin A, $M=84.3 \%, 95 \% \mathrm{CI}$ : [81.2\%, 87.0\%] for Dolphin B, and $M=$ $80.8 \%, 95 \%$ CI: [77.1\%, 84.0\%] for Dolphin C) and lower performance on block $1(M=87.7 \%$, 95\% CI: $[84.9 \%, 90.1 \%])$ vs. block $2(M=94.0 \%, 95 \%$ CI: $[92.3 \%, 95.3 \%])$.

Interview responses. In Experiment 2, 100\% of participants reported hearing a difference between the three dolphins' whistles. Thirty-five of 36 reported hearing a difference in frequency contour, 35 reported a difference in duration, 31 reported a difference in timbre, 27 reported a difference in frequency, 20 reported hearing a difference in amplitude, and 8 reported another difference, such as attributing personality or emotional characteristics to the dolphins' whistles (e.g., Dolphin C was described as more “excited"). Participants in Experiment 2 most commonly reported confusing Dolphins B and C, and participants were most likely to report that frequency contour was one of the most confusing similarities between the whistles. Participants' ratings of how frequently they used each auditory cue to identify the dolphin whistles with and without boat noise present during Experiments 2 are shown in Figure 9. Participants primarily 
used frequency contour, followed by duration, as their most frequent listening cues. Participants assessments of which cue or cues were most helpful for each dolphin during Experiments 2 are given in Table 10. Frequency contour was the most frequently reported "main" cue for all three dolphins, but some cues were used differently for the different dolphins. For example, timbre was reportedly used by 11 participants to identify Dolphin $C$ in the absence of noise, and by 8 in the presence of noise, but was never used to identify Dolphin A, and only used twice for Dolphin B in both noise conditions. Likewise, duration was used to identify Dolphin A and C 17-24 times in either noise condition, but only 10-12 times for Dolphin B.

To test the prediction that participants would use different strategies in the presence and absence of boat noise, participants' ratings of how frequently they used each auditory cue in either noise condition during Experiment 2 were compared using a 2 (absence or presence of boat noise) $\times 6$ (auditory cue: amplitude, frequency, frequency contour, duration, timbre, other ) repeated measures analysis of variance (ANOVA). Mauchly's test indicated that the assumption of sphericity had been violated for auditory cue $\left(\chi^{2}(14)=34.25, p<.001\right)$ and the interaction between absence or presence of boat noise and auditory cue $\left(\chi^{2}(14)=43.29, p<.001\right)$, therefore degrees of freedom were corrected using Greenhouse-Geisser estimates of sphericity for these effects ( $\varepsilon=0.72$ and $\varepsilon=0.63$, respectively).

There was a significant main effect for auditory cue, $F(3.57,125.10)=65.71, p<.001$, but no significant effect for the presence or absence of boat noise, $F(1,35)=0.42, p>.05$. Posthoc analyses of the main effect for cue were conducted using the Bonferroni correction for multiple comparisons (Table 11). Participants reported using frequency contour the most often of any cue followed by duration, whereas amplitude and other cues were used least often. 
There was also a significant interaction effect between the presence of boat noise and auditory cue, $F(3.16,110.43)=2.72, p=.045$. For five auditory cues, there was no difference in how frequently cues were used in the presence vs. absence of boat noise (Table 12). Participants reported using whistle amplitude as a cue significantly more often in the presence of boat noise vs. without boat noise. Thus, participants reportedly used the same listening strategy whether or not boat noise was present except for a single auditory cue.

Strategy analysis. To further investigate the hypothesis that participants would alter their strategies in the presence of boat noise, an analysis was conducted to determine whether participants' reported strategies had any relationship with their performance. Participants' ratings of how frequently they used each cue in the absence or presence of boat noise were compared to their performance under the same conditions. This was intended to test whether participants' cue use affected their performance on the task in the presence or absence of boat noise. If the efficacy of participants' listening strategies varied by noise condition, it could constitute another variation between the use of listening strategies in the presence or absence of boat noise. For each auditory cue, participants' ratings of how frequently they used the cue were compared to their performances using a quadratic curve model of best fit. No curves were found to significantly predict participants' performances based on how frequently they used the auditory cues (all $p$ 's $>0.05$ ). Figure 10 contains a scatter plot of participants accuracies and ratings of how frequently they used each cue in the absence of boat noise, and Figure 11 shows the same, but in the presence of boat noise.

\section{Discussion}

Experiment 2 added the challenge of masking noise and lower-amplitude signals to the signature whistle identification task. Ratio of signal to noise played a major role in participants' 
accuracies. Participant performance under different ratios of signal to noise could be sorted into three main groups. The best performance occurred with low signal and no noise or low noise, or with medium signal and no noise, low noise, or medium noise. Participants performed notably worse under conditions with low signal and medium noise, or medium signal and high noise. Performance neared chance under conditions with low signal and high noise, a condition which might resemble situations in-situ wherein a marine vessel has approached a group of dolphins and two individuals, such as a mother and calf, become separated. Though dolphin performance might vary from that of humans, these results provide evidence that low ratios of signal to noise reduce likelihood of correct whistle identification, which is also likely to occur for dolphins. It is also worth noting that human participants in this experiment always knew that a signature whistle would be played in each trial, and were actively listening for one whenever a sound clip was played. In the ocean, dolphins may be more likely to miss a whistle outright under some of these challenging conditions, thus losing even the opportunity to successfully identify it.

There was also an interaction between boat noise sample and signal to noise ratio (Figure 8). In the easiest conditions, there were no differences between the various noise samples, but in harder conditions, such as low signal with medium or high noise, or medium signal with high noise, differences arose in the participant performance across the five noise samples. Furthermore, there was a relatively clear pattern of which noise samples caused the worst performance in these more challenging conditions. Noise 1 was consistently easier, while noises 3 and 4 were consistently more challenging in conditions with more difficult signal to noise ratios. This seems to be indicative of a plateau, above which minor challenges such as differences in the qualities of masking noise do not seem to affect auditory discrimination of signature whistles. However, when the ratio of signal to noise is lower, and thus the masking is 
more severe, differences in the qualities of the masking noises seem to influence how severely they will mask the signal.

The underlying cause for different performance with different boat noise samples may be explained by the similarity in frequency between the noise and the signal, as hypothesized. There were significant interaction effects between boat noise peak frequency and signal to noise ratio, as well as between boat noise peak frequency and whistle peak frequency. Post-hoc analyses revealed that, in lower signal to noise ratio conditions, it was the boat noises with the highest peak frequencies which tended to most severely disrupt whistle identification. Given that all of the dolphin whistles were higher in frequency than that boat noises, this would indicate that boat noises of more similar frequencies to the whistles would be more negatively impactful on performance. This was confirmed by the analysis of the interaction between boat noise peak frequency and whistle peak frequency. In short, this interaction showed that higher-frequency boat noises paired with lower-frequency whistles both led to poorer performance. In addition, the frequency of the whistles only affected performance when in the presence of higher-frequency boat noises. This supports the hypothesis that similarity in frequency between the whistles and the boat noises would impair whistle identification.

In the interview, participants almost universally cited frequency contour as the most important cue for identifying signature whistles, like in Experiment 1. Frequency contour was reportedly the most common cause of mistaken whistle identity. It had the highest frequency of use, and it was the most frequently cited cue for identifying each individual dolphin's whistle as well, indicating that this either does not vary among dolphins or varies only somewhat (e.g., Branstetter et al., 2016a; Branstetter et al., 2016b). 
There was a clear overall strategy which was fairly consistent among participants. Participants reportedly used frequency contour almost every trial, with duration as a close second. Participants reportedly used frequency and timbre about the same - at slightly less than half of the time. Finally, participants reported that they never or almost-never used amplitude or other cues. The lack of usefulness of amplitude might be deceptive, however, as the artificial manipulation of signal amplitude might have left only change in amplitude over time as an available cue. It is unlikely that this would be different in nature, however, given that perceived amplitude will vary with distance as well as other factors. There was no correlation found between participants' strategies and their success. That said, there was a clear overall strategy which was fairly consistent among participants. Therefore, it is possible that low variation between participants' strategies could account for the lack of significant differences in performance based on differences in strategy. Small differences in strategy may have had undetectable differences in performance. Additionally, participants' reported strategies would only reflect how much or little they tried to use each cue, not how effective they were at using the cues.

There was also no overall reported change in strategy in the absence or presence of noise. It seems that, at least in humans, boat noise does not result in a change of listening strategy when identifying whistles from these three dolphins. This is counterintuitive, given that different ratios of signal to noise resulted in performance varying from $40.83 \%$ to $94.07 \%$. It is therefore possible that strategy is unable to account for this change in difficulty, and that the strategy used (attending primarily to frequency contour) was the best possible strategy regardless of noise condition. It is worth noting that there is little way of knowing from this experiment if the strategy that most participants seemingly came to is, in fact, the ideal strategy for this task. 
Further study with human participants is necessary to perform more in-depth analysis of the efficacy of different strategies.

\section{General Discussion}

Experiment 1 was the first study to test the hypothesis that human listeners can auditorily discriminate among authentic whistles from bottlenose dolphins. The results showed that participants were nearly perfect when it came to discriminating among three dolphins' signature whistles, which strongly supported this hypothesis. During Experiment 1, participants reported that frequency contour and timbre were the most useful cues for identifying signature whistles. Experiment 2 followed the same procedure as Experiment 1, but utilized more challenging conditions for the listening task, including trials with boat noise at eight signal to noise ratios. As predicted, performance decreased when the ratio of signal to noise decreased. In more challenging conditions there were also differences in performance depending on which boat noise sample was played, supporting the hypothesis that acoustic characteristics of masking noise affect its' impact on auditory discrimination. This can most likely be explained by the similarity of the frequencies of the whistle signal and the boat noise. These findings indicate that all of these factors might play a role in how successfully a human is able to identify signature whistles in the presence of noise. In the interview, participants reported no significantly different strategy for trials where noise was present versus trials where it was not, except for a significant but small increase in reported use of amplitude. This contradicted the hypothesis that participants would alter their listening strategies in response to challenging conditions. This might indicate that the ideal signature whistle listening strategy does not change even when noise is present.

The next step in this research is to pursue a follow-up study with dolphins. The findings of the current study serve to establish hypotheses for such a study, and the current study's 
methodology may serve as a useful scaffold for such a follow-up study. However, until these findings are compared with similar research on dolphins, it cannot be assumed that these findings will generalize to dolphins. However, the hypotheses and methods which may be developed based in part on the current research could be instrumental in future work on behavioral and social impacts of anthropogenic noise in dolphins.

This study is consistent with previous studies showing humans can discriminate visually among signature whistles (e.g., Sayigh et al., 2007). It is also consistent with Branstetter and colleagues' (2016b) findings that humans could discriminate auditorily among whistle-like sounds. Furthermore, this study is consistent with previous studies that suggest signature whistles are largely defined by, and therefore identified using, frequency contour. Previous studies have shown that humans and dolphins can discriminate among whistle-like stimuli even when frequency contour is the only differentiating cue among stimuli (Branstetter et al., 2016b), and that frequency contour is critical in visual identification of signature whistles using spectrograms (e.g., Janik, 1999; Janik \& Slater, 1998; Kershenbaum et al., 2013; Sayigh et al., 2007; Watwood et al., 2004). In the present study, frequency contour was used the most often of all auditory cues, and participants also reported that their mistakes largely were the result of specific instances when frequency contour was rendered less useful by masking noise. There were a couple of situations in which this occurred. The first was situations when noise masked parts of the frequency contour, thus forcing participants to either attempt to fill in the parts they had missed, or fall back on other cues. The other situation when frequency contour was less effective was when two dolphins possessed whistles with similar contour. Most participants seemed to agree that the frequency contours of Dolphin B and C's signature whistles were qualitatively similar, and those were the dolphins they most often confused. 
Reductions in signal to noise ratio had a large, negative effect on participant

performance. During some trials at the lowest ratios of signal to noise, participants expressed that they were unable to discern any whistle, despite knowing one was present and attempting to specifically listen for it. This seems to confirm the existing literature on how masking noise affects auditory discrimination in humans (Egan \& Hake, 1950) - lower signal to noise ratios lead to greater difficulty and poorer performance. Furthermore, some masking noises were more impairing than others. There could be multiple factors affecting how impactful different boat noise samples were on whistle identification. Though all of the boat noise recordings were set to play at a certain peak amplitude, that did not prevent lower amplitude parts of some noises from allowing more of the underlying signature whistle to be perceived. For example, more intermittent noise samples were less impactful on participant performance. Qualitatively, boat noise 1 was the most intermittent of the noise samples, and also yielded the highest performance in more challenging conditions. In addition, the peak frequency of the boat noise samples was shown to interact with the peak frequency of the whistle samples in their impact on performance. Specifically, more similar peak frequencies resulted in poorer performance. The present study was conducted on humans, but follows patterns of masking seen across different animal taxa, including fish (Vasconcelos et al., 2007), cetaceans (e.g., Branstetter et al., 2013b), and humans (e.g., Egan \& Hake, 1950).

There are some limitations that should be considered when interpreting the results of this study in terms of how marine mammals might be impacted by anthropogenic noise. Perhaps the most obvious is that humans were tested instead of dolphins. Humans have some perceptual differences which might limit the similarity of their results to those of dolphins. Though humans and dolphins possess different external and middle ear structures, both possess a cochlea, and 
both human and dolphin cochlea function similarly (Ketten, 2000). The greatest difference between human and dolphin inner ears is simply that dolphin cochlea are able to detect sounds of higher frequencies than humans are able to (Herman \& Arbeit, 1972; Thompson \& Herman, 1975; Weir et al., 1977). This is unlikely to affect perception of whistles (the stimuli used in the current study), as these occur within human hearing range. Human and dolphin hearing have many similarities, including threshold of amplitude discrimination (Au, 1993; Green, 1993) and frequency discrimination for tonal stimuli (Herman \& Arbeit, 1972; Thompson \& Herman, 1975; Wier, Jesteadt, \& Green, 1977). On another note, dolphins and humans listen to sound in two different media: water and air, respectively. Fortunately, some of the greatest impacts of this difference are the speed at which sound travels and the rate at which sound attenuates in each medium. Both of these differences are irrelevant for the purposes of this study, as sounds were recorded underwater (Kaplan \& Mooney, 2015) and were played directly into the listeners' ears via headphones. Furthermore, there was no dimension of directionality of this audio to be impacted by the speed of transmission of sound traveling through air instead of water.

Perhaps a greater limitation in extending these results to dolphins is a difference in experience between humans and dolphins - though humans process sounds frequently, often in the presence of masking noise, the actual sounds and masking noises that humans experience are still most likely quite different from those with which dolphins are familiar. With this greater familiarity, it is possible that dolphins would outperform humans on this task. Still, even if this were the case, humans would still serve as a valuable model, as there is no reason to believe that the overall ways in which signals and masking noises interact would be greatly varied between the two species. This would likely at least lead to proportional or analogous changes in performance in dolphins when treated with the same stimuli and noise. Additionally, experience 
(as measured by the two testing blocks in Experiment 2) did not seem to improve performance at the most challenging ratio of signal to noise for the human participants, despite performance improving in all other conditions. This might suggest that even if dolphins are more familiar with both the signals and noises used in the study, this may not factor into their performance on the masked listening task. Experiment 2 only contained two blocks due to the number of other variables and conditions within the task. Thus, each block was contained many trials in which participants could have practiced and improved their performance (although one hour of listening cannot be equated to the hundreds or thousands of hours dolphins likely spend listening to signature whistles). If one considers how quickly participants in Experiment 1 seemed to learn this skill, 240 trials would most likely seem sufficient for participants to become experienced listeners. Therefore, the fact that this experience does not translate to proficiency with whistle identification in the presence of noise is indicative that experience may only go so far in combatting the effects of masking.

There are potential differences between the contexts of human subjects participating in this experiment in a lab versus dolphins experiencing analogous situations in the real world. Dolphins experiencing acute boat noise are likely close to a moving boat and could experience stress because of that and other aspects of the situation. A dolphin trying to acoustically discriminate among signature whistles may be separated from another individual, such as a calf and mother, which could lead to further stress. It is worth noting that stress might increase, decrease, or not directly affect a dolphin's auditory discrimination abilities, depending on how stress affects these specific cognitive processes. On another, similar note, dolphins are more familiar with their own natural vocalizations than a human participant. Dolphins may have biological mechanisms specifically adapted to help comprehend these vocalizations, and, 
regardless, certainly have more experience with discriminating among their species' vocalizations, likely both with and without noise present. Humans possess a similar ability phonemic restoration allows us to use context to mentally replace parts of speech which are masked by noise when we are listening to spoken language (Zimbardo \& Gerrig, 1996). These processes are difficult to account for with current knowledge of dolphin auditory perception, communication, and cognitive abilities. However, the large body of literature on acoustic masking in dolphins would seem to indicate that these processes are fairly similar in both humans and dolphins (Au et al., 1988; Au \& Penner, 1981; Branstetter et al., 2016a; Branstetter et al., 2013a; Lemonds, Au, Vlachos, \& Nachtigal, 2012). A follow-up study on dolphin perception of the same stimuli could reveal the extent to which this study's findings generalize to dolphins.

Another area in which this study was limited is in the number of whistle samples used. Only whistles from three dolphins were utilized as stimuli in this experiment, which does not represent the variety of dolphin signature whistles experienced by a typical dolphin. Future research might expand on this, either by specifically studying a wide range of whistles, or by utilizing fewer additional whistles but in multiple experiments. Another possible pitfall is that the whistles used in this study were from dolphins in managed care. As mentioned earlier, there is evidence that dolphins create slightly different signature whistles for themselves when raised in managed care, which often bear some similarity to sounds present in that environment (Miksis et al., 2002). However, it is unlikely that whistles from dolphins in managed care are so different that whistles from wild dolphins might be affected differently by masking noise.

Similarly, only five samples of boat noise were used in the current study. The boat noise is from an environment which wild dolphins are known to frequent, but due to the nature of data 
collection, it is not known what vessels are responsible for the noises used (Kaplan \& Mooney, 2015). Though the characteristics of these vessels, such as size and speed, are unknown, enough is known about the acoustic characteristics of the noises themselves to make assessments about which other types of boat noise might also be impactful. Due to the relatively shallow location at which the recordings were made (Kaplan \& Mooney, 2015), it can be asserted that the noise samples are unlikely to include noise from larger craft such as cruise ships, commercial fishing vessels, or cargo ships. These other types of noise should be used in a future study to gain further understanding of how more diverse types of anthropogenic noise may differ in their masking effects. Though all of the aforementioned limitations do affect how much on can assume these findings might generalize to dolphins, the target organism, these findings are still valuable in generating hypotheses for future studies on dolphins, and might garner some attention which could affect current regulation of marine anthropogenic noise.

Another aspect of the experiment to consider is the interview. This relied upon selfreport, which can be uninformative or problematic in some circumstances (e.g., Speltz \& Bernstein, 1976; McCambridge, de Bruin, \& Witton, 2012). It may have been difficult for some participants to conceptualize and articulate their listening strategies. It is also possible that some participants' reports of their listening strategies reflected what they expected to use, instead of their actual strategies. Finally, participants may have been subjected to demand characteristics to some extent. For example, participants may have felt an expectation to report differences between their strategies in the presence or absence of noise. This study attempted to utilize several mechanisms to mitigate these problems. Participants were given clear definitions of all terms used to establish a universal understanding of the concepts in question. All interview items were clearly defined, such as repeatedly explaining the different levels of the numerical scales 
used. Questions which would be used for more in-depth analyses, especially participants' ratings of how often they used each stimulus, were placed in the middle of the survey, after several other items pertaining to different auditory cues. This was done intentionally, in order to get participants thinking objectively about their use of different cues before coming up with a numerical representation. Given that no significant differences were found between participants' strategies in the presence or absence of noise, it would appear that these strategies were at least partially successful in reducing demand characteristics.

This study used a sample of human subjects which is much more accessible and inexpensive to test than dolphins. Testing dolphins in human care is difficult due to the limited number of dolphins able to perform long-term research (and the willingness of zoos or aquaria to undertake such research). Training dolphins for new behavioral research procedures can take months to years, and collecting experimental data can take several more months or years. The advantage to testing human subjects is that hypotheses can be generated which would allow more efficient testing of dolphin subjects. First, because humans are faster and less expensive to test than dolphins, and can be tested in greater numbers, this methodology allows for efficient testing of hypotheses. These hypotheses can then be verified using dolphin subjects. In addition, human participants can give feedback about their listening strategies, allowing follow-up studies with dolphins to begin with clear hypotheses about what strategies dolphins, themselves, may be using. To follow up on the current study, a version of this methodology should be repeated with trained dolphins in managed care. Dolphins could be trained to touch one of three choice paddles when they hear one of three corresponding signature whistles. Then, for probe trials, boat noise may be introduced, and samples may include different ratios of signal to noise. For dolphins, all of the variables from this human listening study could be included, as well as the new variable of 
familiar versus unfamiliar signature whistles. For example, a mother dolphin might be more capable of identifying her own signature whistle or that of her calf than an unfamiliar dolphin due to familiarity and practice. It could be informative to know whether or not this familiarity affects the impact of masking noise.

Alternately, a similar study could be adapted to study these phenomena in wild dolphins. Though a similar study with wild dolphins would be challenging, a methodology could be developed similar to Sayigh and colleagues' (1998) study, using head turns to indicate familiarity. This type of study relies on the natural tendency of dolphins to turn their heads towards the source of a familiar signature whistle. This could still be used to test many variables which might impact whistle perception, such as the amplitude and frequency of the masking noise. The effect of familiarity on whistle identification could not be tested in this paradigm because dolphins should exhibit little or no response to any unfamiliar whistles and would not be trained to recognize novel whistles. A benefit of testing wild dolphins would be the ability to show how well the effects of masking noise generalize to different populations. Using humans as model listeners before conducting listening studies in the target species may pave the way for later studies by providing more useful hypotheses and creating more appropriate stimuli. Comparing the performance of subjects across individuals, experience levels, and experimental conditions could grant insight into how the strategies of humans and dolphins vary. In addition, strategy analysis could be performed by restricting the available cues, thus forcing subjects to utilize a specific strategy. For example, in order to test the usefulness of frequency contour and duration, some dolphins could be tested on whistles that do not vary in one of these features.

In order to expand the practical applications of this research, future studies should investigate an even wider variety of noises, including marine construction, sonar, and 
environmental noises such as wind. These studies can also test a wider variety of signals, potentially comparing other types of vocalizations, as well as burst pulses, or vocalizations from other species. Cetaceans are far from the only organisms in the ocean to utilize acoustic signals for communication, and it is likely that all such species are impacted by anthropogenic noise masking their communication. It should also be considered that there are some instances of shared acoustic communication across multiple species, and thus research should also touch upon the communication of species which share their ranges (e.g., Herzing \& Johnson, 1997).

The current study provides evidence that anthropogenic masking noise does impair human perception of dolphin signature whistles. If these findings generalize to dolphins, then even slight disruptions of dolphin communication could have population-scale effects on species fitness. This might be a cause for concern for any regions of the ocean where anthropogenic noise and dolphins are both present. This study indicates that some types of boat noise may be more likely to negatively impact acoustic communication underwater. These findings could be extrapolated to make hypotheses in the future about which species of cetaceans are more likely to be hurt by the masking noises present in their regions. Based on the impact of masking noise from marine vessels on dolphin vocalizations, it seems likely that such noise would disturb the natural social behaviors of dolphins in their natural environment. Such disturbance would clearly be in violation of the Marine Mammal Protection Act (1972). Conservationists and policymakers must determine whether this constitutes a significant threat to marine organisms and, if so, amend the Marine Mammal Protection Act such that the extent to which the act applies to anthropogenic noise is clearly stated. If anthropogenic noise is believed to impact marine mammals, whether at an individual or population level, then guidelines need to be added to the Marine Mammal Protection Act to address this. One possible measure might be to regulate the 
construction of marine vessels to ensure that the amplitude and frequency of the noise they produce is within a range that will have lower masking effect on the species in question. Additionally, boat and ship designers could attempt to engineer vessels and motors which will produce noise at frequencies which will attenuate faster in the marine environment. A potentially beneficial direction for future research would be to determine at what distance anthropogenic noise from any individual boat is likely to disturb the behavior of marine mammals. Such research could help regulate what distance vessels must stay from marine mammals based on the noise they produce. This second measure has considerable limitations, however, as marine mammals may be difficult to spot if they are underwater or if weather conditions reduce visibility, or in areas such as rives and canals where marine mammals and boats must share a more confined space. Given how far some noises travel in the ocean before attenuating, considerable distance may be necessary to avoid disturbing marine mammals.

This study fits into a larger research narrative on the masking effects of anthropogenic noise on a variety of species, not only cetaceans. Another key example in the ocean is the Lusitanian toadfish, which, similarly to the dolphin, uses a special whistle to communicate, wherein the frequency contour is believed to contain identifying information about the individual who produces the whistle (Amorim, Simões, \& Fonseca, 2008). Unlike the signature whistle in dolphins, some study has gone into how this species' whistle perception is impacted by anthropogenic noise, finding that the presence of boat noise greatly increased the threshold at which these toadfish could detect conspecific vocalizations (Vasconcelos, Amorim, \& Ladich, 2007). Humans and dolphins have performed similarly on other listening studies before (e.g., DeLong, 2017), and the current study only uses sounds which are present in a dolphin's natural 
environment, and are therefore likely to be salient to dolphins. This may increase the odds that these findings will generalize well to dolphin subjects.

\section{Conclusion}

This was the first study to show that human listeners can auditorily discriminate among authentic whistles from bottlenose dolphins. The performance of the human participants was impacted by signal to noise ratio and the frequencies of the signal and noise. Listeners primarily relied upon frequency contour when identifying whistles, and reported a significant but small increase in their use of the whistles' amplitude when listening in the presence of boat noise. Human participants continue to be a valuable model organism through which to investigate perceptual processes in dolphins. Human participants are abundant and inexpensive, can be tested quickly, and can verbally answer questions about their strategies. Results of these studies with human listeners can be used to generate hypotheses for studies done with dolphins. These comparative studies, when conducted on both humans and dolphins using the same stimuli and similar procedures, may expedite and augment perceptual research and may provide additional data to improve conservation efforts for dolphins. 


\section{References}

Albuquerque, N. d. S., \& Souto, A. d. S. (2013). Motorboat noise can potentially mask the whistle sound of estuarine dolphins (Sotalia guianensis). Ethnobiology and Conservation, 2, 1-15. doi:10.15451/ec2013-8-2.5-1-15

Amorim, M. C. P., Simões, J. M., \& Fonseca, P. J. (2008). Acoustic communication in the Lusitanian toadfish, Halobatrachus didactylus: Evidence for an unusual large vocal repertoire. Journal of the Marine Biological Association of the United Kingdom, 88(5), 1069-1073. doi:10.1017/S0025315408001677

Au, W. W. L. (1980). Echolocation signals of the Atlantic bottlenose dolphin (Tursiops truncatus) in open waters. In R. G. Busnel and J. F. Fish (Eds.), Animal Sonar Systems (pp. 251-282). New York, United States: Plenum Press.

Au, W. W. L. (1993). The Sonar of Dolphins. Springer-Verlag.

Au, W. W. L., \& Martin, D. W. (1989). Insights into dolphin sonar discrimination capabilities from human listening experiments. The Journal of the Acoustical Society of America, 86(5), 1662-1670. doi:10.1121/1.398596

Au, W. W. L., Moore, P. W. B., \& Pawloski, D. A. (1988). Detection of complex echoes in noise by an echolocating dolphin. The Journal of the Acoustical Society of America, 83(2), 662668. doi:10.1121/1.396161

Au, W. W. L., \& Penner, R. H. (1981). Target detection in noise by echolocating Atlantic bottlenose dolphins. The Journal of the Acoustical Society of America, 70(3), 687-693. doi: $10.1121 / 1.386931$

Benoit-Bird, K. J., \& Au, W. W. L. (2009a). Cooperative prey herding by the pelagic dolphin, Stenella longirostris. The Journal of the Acoustical Society of America, 125(1), 125-137. 
Benoit-Bird, K. J., \& Au, W. W. L. (2009b). Phonation behavior of cooperatively foraging spinner dolphins. The Journal of the Acoustical Society of America, 125(1), 539-546. doi:10.1121/1.2967477

Branstetter, B. K., Bakhtiari, K., Black, A., Trickey, J. S., Finneran, J. J., \& Aihara, H. (2016a). Energetic and informational masking of complex sounds by a bottlenose dolphin (Tursiops truncatus). The Journal of the Acoustical Society of America, 140(3), 19041917. doi:10.1121/1.4962530

Branstetter, B. K., DeLong, C. M., Dziedzic, B., Black, A., \& Bakhtiari, K. (2016b). Recognition of frequency modulated whistle-like sounds by a bottlenose dolphin (Tursiops truncatus) and humans with transformations in amplitude, duration and frequency. PloS One, 11(2), e0147512. doi:10.1371/journal.pone.0147512

Branstetter, B. K., Trickey, J. S., Aihara, H., Finneran, J. J., \& Liberman, T. R. (2013a). Time and frequency metrics related to auditory masking of a $10 \mathrm{kHz}$ tone in bottlenose dolphins (Tursiops truncatus). The Journal of the Acoustical Society of America, 134(6), 4556-4565. doi:10.1121/1.4824680

Branstetter, B. K., Trickey, J. S., Bakhtiari, K., Black, A., Aihara, H., \& Finneran, J. J. (2013b). Auditory masking patterns in bottlenose dolphins (Tursiops truncatus) with natural, anthropogenic, and synthesized noise. The Journal of the Acoustical Society of America, 133(3), 1811-1818. doi:10.1121/1.4789939

Buckstaff, K. C. (2004). Effects of watercraft noise on the acoustic behavior of bottlenose dolphins, Tursiops truncatus, in Sarasota Bay, Florida. Marine Mammal Science, 20(4), 709-725. doi:10.1111/j.1748-7692.2004.tb01189.x 
David, J. A. (2006). Likely sensitivity of bottlenose dolphins to pile-driving noise. Water and Environment Journal, 20(1), 48-54. doi:10.1111/j.1747-6593.2005.00023.x

DeLong, C. M. (2017). Human listening experiments provide insight into cetacean auditory perception. Proceedings of Meetings on Acoustics, 29(1), 010001. doi:10.1121/2.0000447

DeLong, C. M., Au, W. W. L., Harley, H. E., Roitblat, H. L., \& Pytka, L. (2007a). Human listeners provide insights into echo features used by dolphins (Tursiops truncatus) to discriminate among objects. Journal of Comparative Psychology, 121(3), 306-319. doi:10.1037/0735-7036.121.3.306

DeLong, C. M., Au, W. W. L., \& Stamper, S. A. (2007b). Echo features used by human listeners to discriminate among objects that vary in material or wall thickness: Implications for echolocating dolphins. The Journal of the Acoustical Society of America, 121(1), 605617. doi:10.1121/1.2400848

DeLong, C. M., Heberle, A. L., Wisniewski, M. G., \& Mercado, E. (2014). The ability to recognize objects from bottlenose dolphin (Tursiops truncatus) echoes generalizes across multiple orientations in humans and neural networks. Animal Cognition, 17(3), 543-557. doi:10.1007/s 10071-013-0685-0

Egan, J. P., \& Hake, H. W. (1950). On the masking pattern of a simple auditory stimulus. The Journal of the Acoustical Society of America, 22(5), 622-630. doi:10.1121/1.1906661

Fish, J. F., Johnson, C. S., \& Ljungblad, D. K. (1976). Sonar target discrimination by instrumented human divers. Journal of the Acoustical Society of America, 59(3), 602606. doi:10.1121/1.380905

Fripp, D., Owen, C., Quintana-Rizzo, E., Shapiro, A., Buckstaff, K., Jankowski, K., ... Tyack, P. L. (2005). Bottlenose dolphin (Tursiops truncatus) calves appear to model their 
signature whistles on the signature whistles of community members. Animal Cognition, 8(1), 17-26. doi:10.1007/s10071-004-0225-z

Gorman, R. P., \& Sawatari, T. (1985). The use of multidimensional perceptual models in the selection of sonar echo features. The Journal of the Acoustical Society of America, 77(3), 1178-1184. doi:10.1121/1.392182

Green, D. M. (1960). Psychoacoustics and detection theory. The Journal of the Acoustical Society of America, 32(10), 1189-1203. doi:10.1121/1.1907882

Green, D. M. (1993). Auditory intensity discrimination. In W. A. Yost, A. N. Popper, \& R. R. Fay (Eds.), Human Psychophysics (pp. 13-55). New York, NY: Springer New York.

Gridley, T., Cockcroft, V., Hawkins, E. R., Lemon Blewitt, M., Morisaka, T., \& Janik, V. M. (2014). Signature whistles in free ranging populations of Indo-Pacific bottlenose dolphins, Tursiops aduncus. Marine Mammal Science, 30(2), 512-527.

doi:10.1111/mms. 12054

Harley, H. E. (2008). Whistle discrimination and categorization by the Atlantic bottlenose dolphin (Tursiops truncatus): A review of the signature whistle framework and a perceptual test. Behavioural Processes, 77(2), 243-268. doi:10.1016.j.beproc.2007.11.002

Hawkins, J. E., \& Stevens, S. S. (1950). The masking of pure tones and of speech by white noise. The Journal of the Acoustical Society of America, 22(1), 6-13. doi:10.1121/1.1906581

Helweg, D. A., Roitblat, H. L., Nachtigall, P. E., Au, W. W. L., \& Irwin, R. J. (1995). Discrimination of echoes from aspect-dependent targets by a bottlenose dolphin and human listeners. In R. A. Kastelein, J. A. Thomas, \& P. E. Nachtigall (Eds.), Sensory 
Systems of Aquatic Mammals (pp. 129-136). Woerden, The Netherlands: De Spil Publishers.

Herman, L. M., \& Arbeit, W. R. (1972). Frequency difference limens in the bottlenose dolphin: 1-70 kc/s. Journal of Auditory Research, 2, 109-120.

Herzing, D. L. (1996). Vocalizations and associated underwater behavior of free-ranging Atlantic spotted dolphins, Stenella frontalis and bottlenose dolphins, Tursiops truncatus. Aquatic Mammals, 22, 61-80.

Herzing, D. L., \& Johnson, C. M. (1997). Interspecific interactions between Atlantic spotted dolphins (Stenella frontalis) and bottlenose dolphins (Tursiops truncatus) in the Bahamas, 1985-1995. Aquatic Mammals, 23, 85-99.

Holm, S. (1979). A simple sequentially rejective multiple test procedure. Scandinavian Journal of Statistics, 6(2), 65-70.

Janik, V. M. (1999). Pitfalls in the categorization of behaviour: A comparison of dolphin whistle classification methods. Animal Behaviour, 57(1), 133-143. doi:10.1006/anbe.1998.0923

Janik, V. M., Dehnhardt, G., \& Todt, D. (1994). Signature whistle variations in a bottlenosed dolphin, Tursiops truncatus. Behavioral Ecology and Sociobiology, 35, 243-248.

Janik, V. M., King, S. L., Sayigh, L. S., \& Wells, R. S. (2013). Identifying signature whistles from recordings of groups of unrestrained bottlenose dolphins (Tursiops truncatus). Marine Mammal Science, 29(1), 109-122. doi:10.1111/j.1748-7692.2011.00549.x

Janik, V. M., \& Sayigh, L. S. (2013). Communication in bottlenose dolphins: 50 years of signature whistle research. Journal of Comparative Physiology A: Neuroethology, Sensory, Neural, and Behavioral Physiology, 199, 479-489. doi:10.1007/s00359-0130817-7 
Janik, V. M., Sayigh, L. S., \& Wells, R. S. (2006). Signature whistle shape conveys identity information to bottlenose dolphins. Proceedings of the National Academy of Sciences, 103(21), 8293-8297. doi:10.1073/pnas.0509918103

Janik, V. M., \& Slater, P. J. (1998). Context-specific use suggests that bottlenose dolphin signature whistles are cohesion calls. Animal Behaviour, 56(4), 829-838.

Janik, V. M., \& Thompson, P. M. (1996). Changes in surfacing patterns of bottlenose dolphins in response to boat traffic. Marine Mammal Science, 12, 597-602.

Jon, S. N., Lutz, J., Maria-Liisa, G.-R., \& Wilhelm, M.-G. H. (1999). Influence of acoustic masking noise in $\mathrm{PMRI}$ of the auditory cortex during phonetic discrimination. Journal of Magnetic Resonance Imaging, 9(1), 19-25. doi:doi:10.1002/(SICI)15222586(199901)9:1<19::AID-JMRI3>3.0.CO;2-K

Kaplan, M. B., \& Mooney, T. A. (2015). Ambient noise and temporal patterns of boat activity in the US Virgin Islands National Park. Marine Pollution Bulletin, 98(1), 221-228. doi:10.1016/j.marpolbul.2015.06.047

Kershenbaum, A., Sayigh, L. S., \& Janik, V. M. (2013). The encoding of individual identity in dolphin signature whistles: How much information is needed? PloS One, 8(10), e 77671. doi:10.1371/journal.pone.0077671

Ketten, D. R. (2000). Cetacean Ears. In W. W. L. Au, R. R. Fay, \& A. N. Popper (Eds.), Hearing by Whales and Dolphins (pp. 43-108). New York, NY: Springer New York.

King, S. L., \& Janik, V. M. (2013). Bottlenose dolphins can use learned vocal labels to address each other. Proceedings of the National Academy of Sciences of the United States of America, 110(32), 13216-13221. doi:10.1073/pnas.1304459110 
King, S. L., Sayigh, L. S., Wells, R. S., Fellner, W., \& Janik, V. M. (2013). Vocal copying of individually distinctive signature whistles in bottlenose dolphins. Proceedings of the Royal Society B: Biological Sciences, 280(1757), 20130053. doi:10.1098/rspb.2013.0053

Lemonds, D. W., Au, W. W. L., Vlachos, S. A., \& Nachtigall, P. E. (2012). High-frequency auditory filter shape for the Atlantic bottlenose dolphin. The Journal of the Acoustical Society of America, 132(2), 1222-1228. doi:10.1121/1.4731212

Lusseau, D. (2003). Effects of tour boats on the behavior of bottlenose dolphins: Using Markov chains to model anthropogenic impacts. Conservation Biology, 17(6), 1785-1793. doi:10.1111/j.1523-1739.2003.00054.x

Marshall, L., \& Jesteadt, W. (1986). Comparison of pure-tone audibility thresholds obtained with audiological and two-interval forced-choice procedures. Journal of Speech, Language, and Hearing Research, 29(1), 82-91. doi:10.1044/jshr.2901.82

Marine Mammal Protection Act, 16 USC $\S \S 1361$ et seq. (1972).

McCambridge, J., de Bruin, M., \& Witton, J. (2012). The effects of demand characteristics on research participant behaviours in non-laboratory settings: A systematic review. PloS One, 7(6), e39116. doi:10.1371/journal.pone.0039116

Mercado, E., III. \& DeLong, C. M. (2010). Dolphin cognition: Representations and processes in perception and memory. International Journal of Comparative Psychology, 23, 344-378.

Miksis, J. L., Tyack, P. L., \& Buck, J. R. (2002). Captive dolphins, Tursiops truncatus, develop signature whistles that match acoustic features of human-made model sounds. The Journal of the Acoustical Society of America, 112(2), 728-739. doi:10.1121/1.1496079 
Muller-Gass, A., Marcoux, A., Logan, J., \& Campbell, K. B. (2001). The intensity of masking noise affects the mismatch negativity to speech sounds in human subjects. Neuroscience Letters, 299(3), 197-200. doi:10.1016/S0304-3940(01)01508-7

Nelken, I., Rotman, Y., \& Yosef, O. B. (1999). Responses of auditory-cortex neurons to structural features of natural sounds. Nature, 397, 154-157. doi:10.1038/16456

Overstrom, N. A. (1983). Association between burst-pulse sounds and aggressive behavior in captive Atlantic bottlenosed dolphins (Tursiops truncatus). Zoo Biology, 2(2), 93-103. doi:10.1002/zoo.1430020203

Pirotta, E., Merchant, N. D., Thompson, P. M., Barton, T. R., \& Lusseau, D. (2015). Quantifying the effect of boat disturbance on bottlenose dolphin foraging activity. Biological Conservation, 181, 82-89. doi:10.1016/j.biocon.2014.11.003

Quick, N. J., \& Janik, V. M. (2008). Whistle rates of wild bottlenose dolphins (Tursiops truncatus): Influences of group size and behavior. Journal of Comparative Psychology, 122(3), 305-311. doi:10.1037/0735-7036.122.3.305

Quick, N. J., \& Janik, V. M. (2012). Bottlenose dolphins exchange signature whistles when meeting at sea. Proceedings of the Royal Society B: Biological Sciences, 279(1738), 2539-2545. doi:10.1098/rspb.2011.2537

Raudenbush, S. W., \& Bryk, A. S. (2002). Hierarchical Linear Models: Applications and Data Analysis Methods. Sage.

Ross, D. (2005). Ship sources of ambient noise. IEEE Journal of Oceanic Engineering, 30(2), 257-261. doi:10.1109/JOE.2005.850879 
Sayigh, L. S., Esch, H. C., Wells, R. S., \& Janik, V. M. (2007). Facts about signature whistles of bottlenose dolphins, Tursiops truncatus. Animal Behaviour, 74(6), 1631-1642. doi:10.1016/j.anbehav.2007.02.018

Sayigh, L. S., \& Janik, V. M. (2010). Dolphin signature whistles. In M. D. Breed \& J. Moore (Eds.), Encyclopedia of Animal Behavior (pp. 553-561). Oxford: Academic Press.

Sayigh, L. S., Tyack, P. L., Wells, R. S., \& Scott, M. D. (1990). Signature whistles of freeranging bottlenose dolphins Tursiops truncatus: Stability and mother-offspring comparisons. Behavioral Ecology and Sociobiology, 26(4), 247-260. doi:10.1007/BF00178318

Sayigh, L. S., Tyack, P. L., Wells, R. S., Scott, M. D., \& Irvine, A. B. (1995). Sex difference in signature whistle production of free-ranging bottlenose dolphins, Tursiops truncates. Behavioral Ecology and Sociobiology, 36(3), 171-177. doi:10.1007/bf00177793

Sayigh, L. S., Tyack, P. L., Wells, R. S., Solow, A. R., Scott, M. D., \& Irvine, A. B. (1999). Individual recognition in wild bottlenose dolphins: A field test using playback experiments. Animal Behaviour, 57(1), 41-50. doi:10.1006/anbe.1998.0961

Sayigh, L. S., Wells, R. S., \& Janik, V. M. (2017). What's in a voice? Dolphins do not use voice cues for individual recognition. Animal Cognition, 20(6), 1067-1079. doi:10.1007/s 10071-017-1123-5

Smolker, R., Mann, J., \& Smuts, B. (1993). Use of signature whistles during separations and reunions by wild bottlenose dolphin mothers and infants. Behavioral Ecology and Sociobiology, 33(6), 393-402. 
Speltz, M. L., \& Bernstein, D. A. (1976). Sex differences in fearfulness: Verbal report, overt avoidance and demand characteristics. Journal of Behavior Therapy and Experimental Psychiatry, 7(2), 117-122. doi:10.1016/0005-7916(76)90067-7

Thompson, R. K. R., \& Herman, L. M. (1975). Underwater frequency discrimination in the bottlenosed dolphin (1-140 kHz) and the human (1-8 kHz). The Journal of the Acoustical Society of America, 57(4), 943-948. doi:10.1121/1.380513

Tukey, J. (1949). Comparing individual means in the analysis of variance. Biometrics, 5(2), 99114.

Vasconcelos, R. O., Amorim, M. C. P., \& Ladich, F. (2007). Effects of ship noise on the detectability of communication signals in the Lusitanian toadfish. Journal of Experimental Biology, 210(12), 2104-2112. doi:10.1242/jeb.004317

Watwood, S. L., Tyack, P. L., \& Wells, R. S. (2004). Whistle sharing in paired male bottlenose dolphins, Tursiops truncatus. Behavioral Ecology and Sociobiology, 55(6), 531-543. doi:10.1007/s00265-003-0724-y

Weilgart, L. S. (2007). The impacts of anthropogenic ocean noise on cetaceans and implications for management. Canadian Journal of Zoology, 85(11), 1091-1116. doi:10.1139/Z07-101

Wier, C. C., Jesteadt, W., \& Green, D. M. (1977). Frequency discrimination as a function of frequency and sensation level. The Journal of the Acoustical Society of America, 61(1), 178-184. doi:10.1121/1.381251

Würsig, B., \& Richardson, W. J. (2009). Noise, Effects of. In Encyclopedia of Marine Mammals (Second Edition) (pp. 765-773). London: Academic Press.

Zimbardo, P. G., \& Gerrig, R. J. (1996). Perception. In Psychology and Life, 14 (pp. 258-302). New York, NY: HarperCollins College Publishers. 
Appendix A

Participant Information Form

Dolphin Whistle Study

ID:

Date Tested:

Age:

Gender:

Have you ever participated in any psychoacoustic/hearing studies? (describe)

\section{Part 1: Musical Ability}

1a. Do you play an instrument or sing?

1b. Which instrument?

2. Rate your ability level

( 1 = very little ability/proficiency, $4=$ moderate ability/proficiency, $7=$ expert/professional level, please circle one)

2

\section{Part 2: Hearing Profile}

1. Has a professional audiologist or doctor ever diagnosed you as having a hearing problem? (give date)

2. Do you have trouble hearing any types of sounds? (if yes, list)

\section{Part 3: Noise Exposure}

1a. Do you work in an environment with a lot of background noise?

1b. If yes, how often does your work require careful listening while noise is happening?

$(1=$ once per day or less, $4=$ once per hour, $7=$ nearly constantly, please circle one $)$

$\begin{array}{llllllll}1 & 2 & 3 & 4 & 5 & 6 & 7\end{array}$

2a. Do you work in an environment that requires you to wear hearing protection?

2b. If yes, how well does the hearing protection block loud sounds?

( 1 = not at all, $4=$ blocks about half of sounds, $7=$ completely, please circle one $)$ 


\section{Appendix A (continued)}

3a. How frequently do you listen to music?

$(1=$ less than once per week, $4=$ once every 1-2 days, $7=$ near-constantly, please circle one $)$

$\begin{array}{llllllll}1 & 2 & 3 & 4 & 5 & 6 & 7\end{array}$

3b. How do you listen to music most of the time? (please circle one)

$\begin{array}{ccccc} & \text { Using in-ear } & \text { Using over-ear } & \text { Using a speaker } & \\ \text { In the car } & \text { headphones (earbuds) } & \text { headphones } & \text { (including } & \text { Other } \\ & & & \text { computer speakers) }\end{array}$

3c. What volume do you listen to music at, most of the time?

$(1$ = minimum possible volume, 4 = half of maximum volume, 7 = maximum possible volume, please circle one) 


\section{Appendix $B$ \\ Listening Task Training Script \\ Signature Whistle Listening Task Introduction}

Before you start the listening task, I'll tell you a little bit more about the subject of the study, and about the task you'll be doing.

First, you should know that dolphins use a variety of sounds to communicate, including whistles known as "signature whistles." Each dolphin has a unique signature whistle, somewhat like human names. This means that if you learn to recognize a dolphin's signature whistle, you can identify that dolphin using nothing but the sound of that whistle.

In this study, you will listen to signature whistles from three dolphins. First, you'll get to listen to one whistle from each dolphin, and you may ask for this whistle to be repeated until you to become familiar with all three dolphins and their signature whistles.

Ready to start with the training?

\section{Signature Whistle Listening Task Training}

The following samples contain signature whistles from three dolphins, which we will call "Dolphin A," "Dolphin B," and "Dolphin C." I'll play all three whistles three times for you. After that, you may request to listen to these samples as many times as you like, until you feel like you could differentiate between all three signature whistles. Try to note specific features of the signature whistles which might help you differentiate later on. As a reminder, if the volume is ever uncomfortably high, or if you need to for any other reason, please take your headphones off and tell me as soon as possible. Put on your headphones now, and I'll play you examples of each dolphin's signature whistle. 


\section{Appendix B (continued)}

\section{Signature Whistle Listening Task Instructions}

Next you'll be tested on your ability to identify the dolphins by their whistles. For each trial, I'll play a whistle for you, then you'll tell me which dolphin you think the whistle belongs to. Throughout this task, I'd like you to pay attention to which auditory cues you're noticing and identifying the dolphins with. After the test, I'll give you a quick interview about what strategies you used to distinguish between the whistles.

For each trial, I'll play one dolphin's signature whistle for you. After the trial is complete, I'd like you to say which dolphin you think produced the whistle, and I will tell you whether your choice was correct or wrong. For some trials, there will be other noise present along with the signature whistle. You can only listen to each trial once, so if you aren't sure, just make your best guess. Once I've told you correct or wrong, I'll begin the next trial.

In order to avoid distractions, you should either close your eyes or focus on the fixation cross on the door, unless you are referencing the vocabulary sheet. The task should take about 30 minutes to complete. Please put on your headphones, and I'll begin the signature whistle listening task. 


\section{Appendix $C$}

\section{Terms and Definitions Handout}

Amplitude, or loudness: how loud or quiet a sound is (aka. volume, intensity). For example: if a car honks its horn as it approaches you, the volume of the horn sound will increase as the car grows nearer. This increase in volume is also known as increasing amplitude.
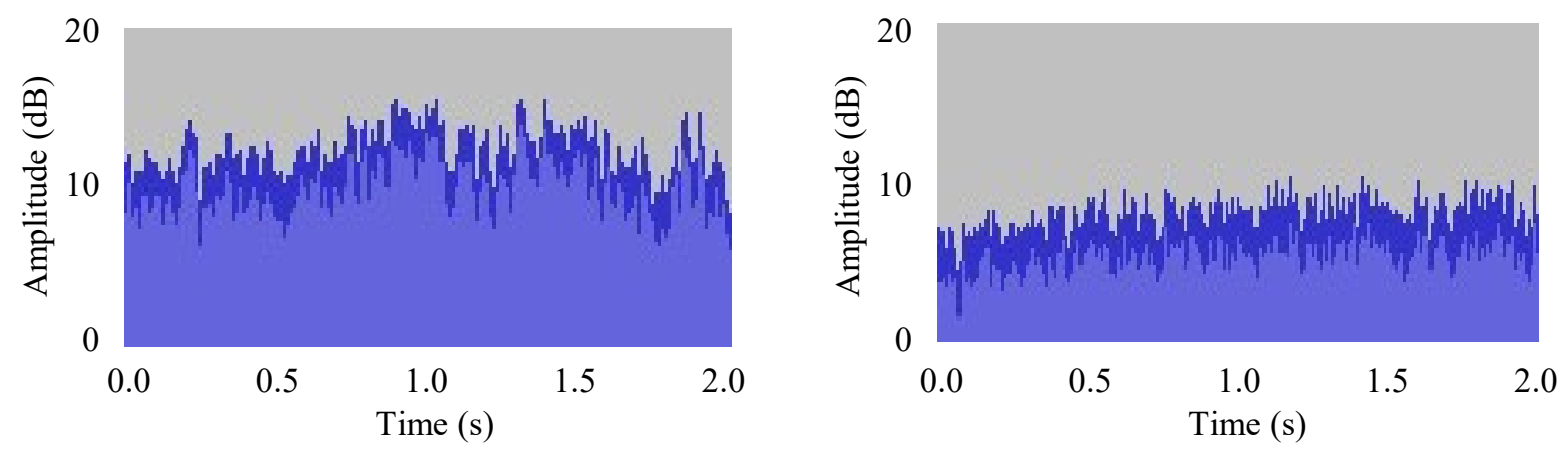

Frequency, or pitch: overall, how high or low a sound is.
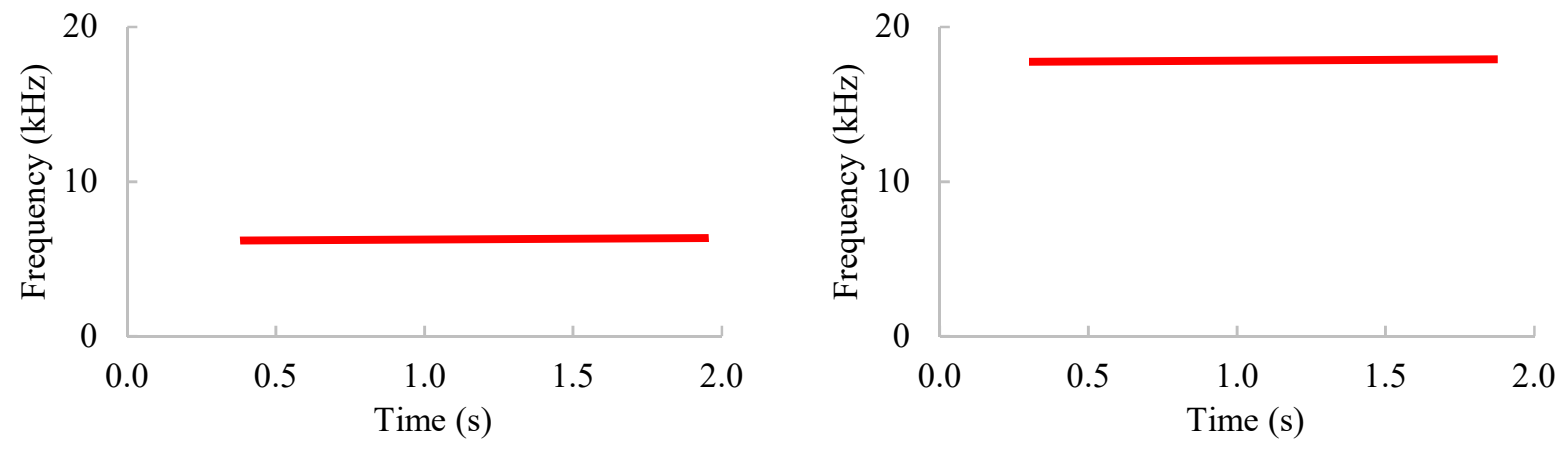

Frequency contour: The profile of change in a sound's frequency (pitch) over time. Frequency contour describes how a sound changes in pitch over the course of the sound.
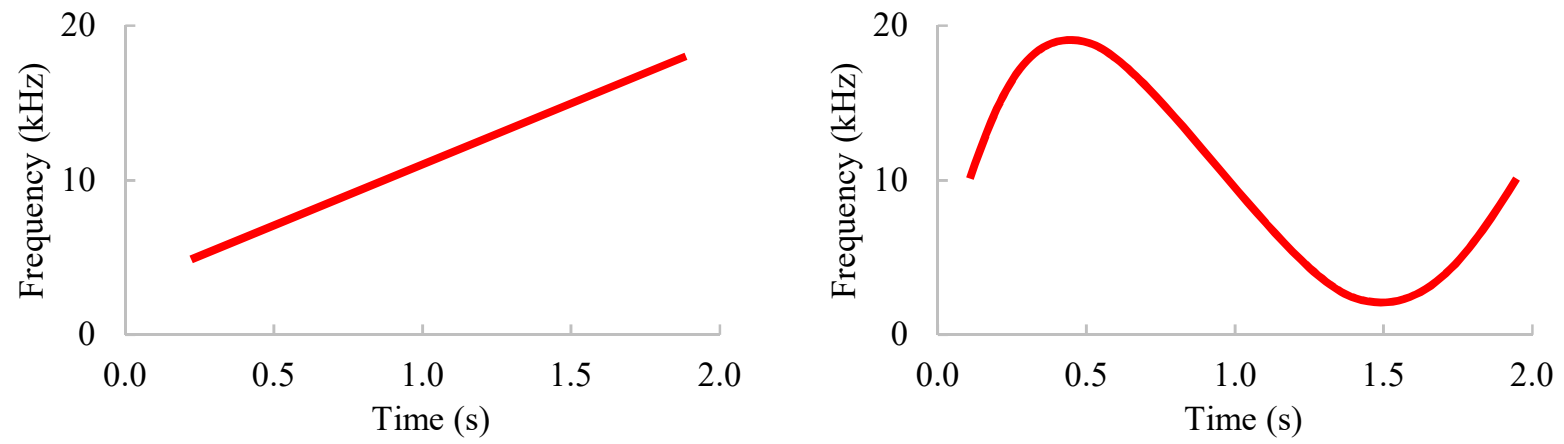


\section{Appendix C (continued)}

Duration, or length: how long a sound lasts.
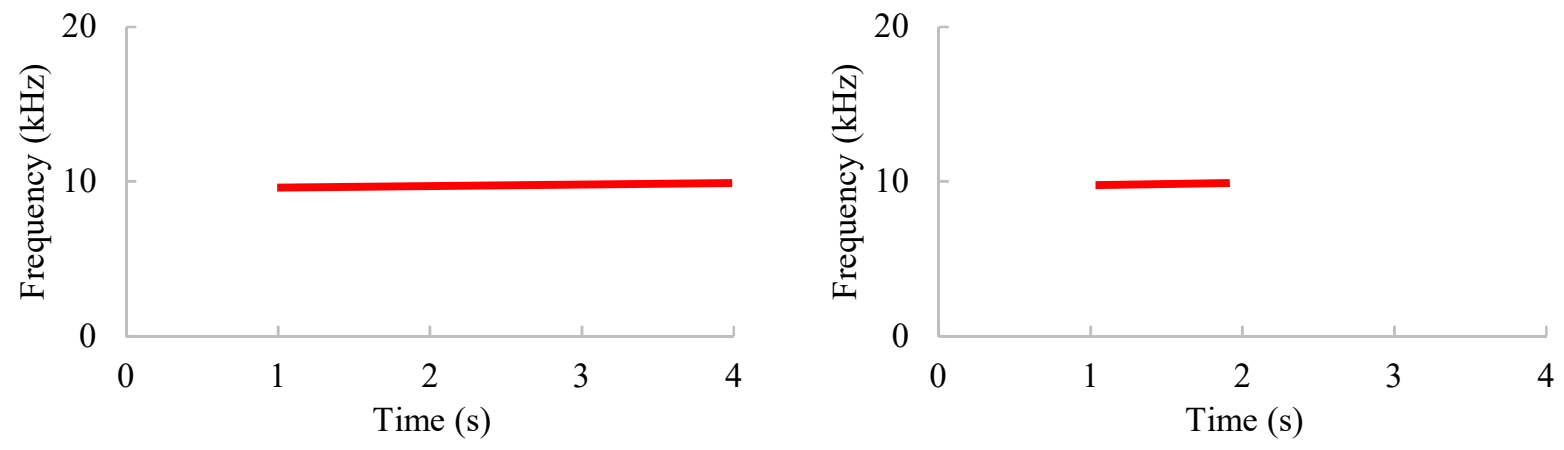

Timbre, or sound quality: the quality of a sound that allows it to be distinguished from sounds of the same pitch, loudness, and duration, such as the difference between how various instruments sound when played (aka. tone color). For example: if you play middle $\mathrm{C}$ on a violin and on a trumpet at the same amplitude, you will hear a difference in the sound quality even though the sounds are played at the same frequency and amplitude.
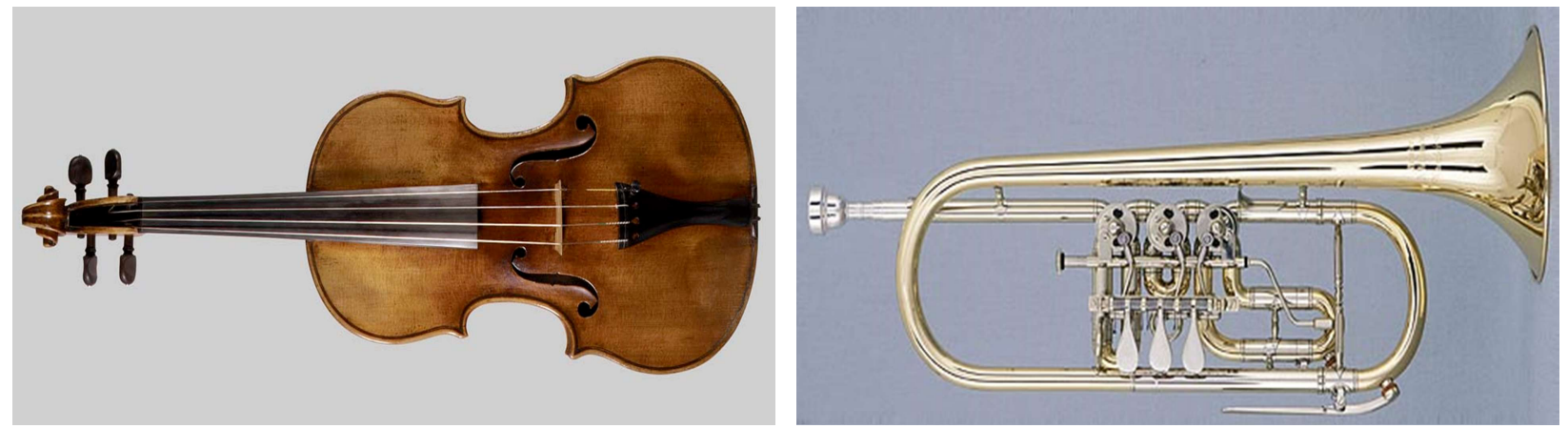

Other features: There may be other sound features which you identify and utilize when distinguishing between signature whistles. You are encouraged to take note of these features as well and explain them during the interview. 


\section{Appendix D}

\section{End of Experiment Interview}

\section{UNPLUG HEADPHONES. PLAY BASELINE SOUNDS AT FULL VOLUME. (EXP. 1)}

\section{Did you hear any differences between the signature whistles of the three dolphins?}

Yes

2. Did you hear a difference in

$\begin{array}{lllll}\text { Amplitude } & \text { Frequency } & \begin{array}{c}\text { Frequency } \\ \text { Contour }\end{array} & \text { Duration } & \text { Timbre }\end{array}$

\section{Other:}

If a difference in amplitude was heard, ask the participant to rank the sounds in terms of loudness ( 1 = loudest, 3 = quietest)

Dolphin A Dolphin B Dolphin C

If a difference in frequency was heard, ask the participant to rank the sounds in terms of pitch ( 1 = highest pitch, 3 = lowest pitch)

Dolphin A Dolphin B Dolphin C

If a difference in frequency contour was heard, ask the participant to describe the sounds in terms of frequency contour (can suggest terms like upsweep and loop)

Dolphin A Dolphin B Dolphin C

If a difference in duration was heard, ask the participant to rank the sounds in terms of duration (1 = shortest, 3 = longest)

Dolphin A Dolphin B Dolphin C

If a difference in timbre was heard, ask the participant to describe

Dolphin A Dolphin B Dolphin C 


\section{Appendix D (continued)}

3. Which pair of whistles do you think you confused most often (in other words, which pair of whistles had the most similar sounds)? How were those whistles similar?
Dolphin A
Dolphin B
Dolphin C

How were they similar?
Amplitude
Frequency
Frequency
Contour
Duration
Timbre
Other

Other:

4. Did you pay attention to different cues with each dolphin's whistle?

Dolphin A:

Amplitude Frequency $\begin{gathered}\text { Frequency } \\ \text { Contour }\end{gathered}$ Duration Timbre $\quad$ Other

Dolphin B:

$\begin{array}{lllll}\text { Amplitude Frequency } & \begin{array}{c}\text { Frequency } \\ \text { Contour }\end{array} \text { Duration } & \text { Timbre } & \text { Other }\end{array}$

Dolphin C:

Amplitude Frequency $\begin{gathered}\text { Frequency } \\ \text { Contour }\end{gathered}$ Duration Timbre $\quad$ Other 


\section{Appendix D (continued)}

5. Overall, how often did you use each cue? Give me a number from 1 to 7 where 1 means you never used the cue, 4 means you used it for about half of the trials, and 7 means you used it every trial.

Amplitude:

1

2

3

4

5

6

7

Frequency

1

2

3

4

5

6

7

Frequency Contour

1

2

3

4

5

6

7

Duration

1

2

3

4

5

6

7

Timbre

1

2

3

4

5

6

7

Other

1

2

3

4

5

6

7

6. Overall, was the task of telling the difference between whistles easy or difficult? Give me a number from 1 to 7 where 1 is the easiest and 7 is the most difficult. 


\section{Appendix E}

Interview Part 2 (Experiment 2 only)

\section{PLAY BASELINE \& MASKED SOUNDS (PILOT, EXP. 2)}

7. Overall, was the task of telling the difference between signature whistles easy or difficult when there was other noise present (compared to where you had to tell the difference between signature whistles without noise)? Give me a number from 1 to 7 where 1 is the easiest and 7 is the most difficult. Again, this is only for when additional noise was played at the same time as the whistles.

1

2

3

4

5

6

8. Overall, how often would you say you used each cue was when background noise was present? Give me a number from 1 to 7 where 1 means you never used the cue, 4 means you used it for about half of the trials, and 7 means you used it every trial.

Amplitude:

1

\section{Frequency}

1

Frequency Contour

1

Duration

1

Timbre

1

Other

2
3

4

4

4

5

6

7 


\section{Appendix E (continued)}

9. In this experiment, the signature whistles were sometimes played with other noise present. Did you have to change the cues you listened to in response to the noise?

\section{Dolphin A:}

$\begin{array}{llll}\text { Amplitude Frequency } & \begin{array}{c}\text { Frequency } \\ \text { Contour }\end{array} \text { Duration } & \text { Timbre }\end{array}$

\section{Dolphin B:}

$\begin{array}{llll}\text { Amplitude Frequency } & \begin{array}{c}\text { Frequency } \\ \text { Contour }\end{array} \text { Duration } & \text { Timbre }\end{array}$ Dolphin C:

Amplitude Frequency $\begin{gathered}\text { Frequency } \\ \text { Contour }\end{gathered}$ Duration Timbre Other


Table 1

Experiment 1 Performance by Test Block

\begin{tabular}{lccc}
\hline & Mean & \multicolumn{2}{c}{$95 \%$ Confidence Interval } \\
\cline { 2 - 4 } Block & Performance (\%) & Lower Bound (\%) & Upper Bound (\%) \\
\hline 1 & 91.43 & 87.63 & 95.22 \\
2 & 97.62 & 95.55 & 99.69 \\
3 & 98.10 & 96.24 & 99.95 \\
4 & 98.57 & 96.96 & 100.18 \\
5 & 99.05 & 97.73 & 100.36 \\
6 & 99.52 & 98.59 & 100.46 \\
7 & 100.00 & 100.00 & 100.00 \\
8 & 99.05 & 97.73 & 100.36 \\
9 & 100.00 & 100.00 & 100.00 \\
10 & 100.00 & 100.00 & 100.00 \\
11 & 100.00 & 100.00 & 100.00 \\
12 & 99.52 & 98.59 & 100.46 \\
13 & 99.52 & 98.59 & 100.46 \\
14 & 100.00 & 100.00 & 100.00 \\
15 & 99.52 & 98.59 & 100.46 \\
16 & 99.52 & 98.59 & \\
\hline$N o t e$. Each & &
\end{tabular}

Note. Each block contained 15 trials: all 5 exemplars from each of the 3 dolphins. Trial order was randomized within each test block and for each participant. 
Table 2

Cues Reported Most Useful for Identifying Each Dolphin in Experiment 1

\begin{tabular}{lllll}
\hline \multirow{2}{*}{ Whistle cues } & \multicolumn{4}{l}{ Number of times reported used per dolphin } \\
\cline { 2 - 5 } & Dolphin A & Dolphin B & Dolphin C & Sum \\
\hline Amplitude & 2 & 2 & 1 & 5 \\
Frequency & 0 & 2 & 2 & 4 \\
Frequency contour & 11 & 14 & 10 & 35 \\
Duration & 9 & 2 & 3 & 14 \\
Timbre & 0 & 1 & 7 & 8 \\
Other & 0 & 0 & 0 & 0 \\
Sum & 22 & 21 & 23 & 66 \\
\hline
\end{tabular}

Note. Participants $(\mathrm{N}=14)$ were allowed to select one or two cues per dolphin. They were encouraged to give a single cue if there was a specific cue which was clearly the most useful for identifying the dolphin in question, or to give two cues if there were multiple cues that were used to identify that dolphin. 
Table 3

Samples of Boat Noise

\begin{tabular}{|c|c|c|c|c|}
\hline Sample & $\begin{array}{l}\text { Date and Time of } \\
\text { Recording (UTC) }\end{array}$ & $\begin{array}{l}\text { Peak Amplitude } \\
\text { (dB) }\end{array}$ & $\begin{array}{c}\text { Peak Frequency } \\
(\mathrm{Hz})\end{array}$ & $\begin{array}{c}\text { Peak Amplitude of } \\
\text { Peak Frequency (dB) }\end{array}$ \\
\hline N0 & $\begin{array}{c}\text { July 7, } 2016 \\
17: 30: 02\end{array}$ & -20.310 & 170 & -48.0 \\
\hline N1 & $\begin{array}{c}\text { May 30, } 2016 \\
21: 20: 02\end{array}$ & -18.771 & 63 & -44.4 \\
\hline $\mathrm{N} 2$ & $\begin{array}{c}\text { June 27, } 2016 \\
\text { 19:30:02 }\end{array}$ & -18.913 & 186 & -34.0 \\
\hline N3 & $\begin{array}{c}\text { July 3, } 2016 \\
15: 20: 02\end{array}$ & -16.250 & 233 & -42.7 \\
\hline N4 & $\begin{array}{c}\text { July 7, } 2016 \\
16: 60: 02\end{array}$ & -11.995 & 132 & -33.8 \\
\hline N5 & $\begin{array}{c}\text { July 11, } 2016 \\
17: 50: 02\end{array}$ & -10.867 & 166 & -36.8 \\
\hline
\end{tabular}

Note. UTC $=$ Coordinated Universal Time. The local time where recordings were collected was 4 hours behind UTC time. Peak amplitude of peak frequency is the maximum amplitude, in decibels, at the frequency which achieves the greatest amplitude over the duration of the noise sample. 
Table 4

Amplitudes of Signature Whistle and Boat Noise in Experiment 2

\begin{tabular}{llc}
\hline $\begin{array}{l}\text { Peak Amplitude of } \\
\text { Signature Whistles }\end{array}$ & Peak Amplitude of Boat & \\
$(\mathrm{dB})$ & Noise & Ratio of signal to noise \\
\hline 50 & N/A & Low signal, no noise \\
& 60 & Low signal, low noise \\
& 65 & Low signal, medium noise \\
& 70 & Low signal, high noise \\
60 & N/A & Medium signal, no noise \\
& 60 & Medium signal, low noise \\
& 65 & Medium signal, medium noise \\
& 70 & Medium signal, high noise \\
\hline
\end{tabular}

Note. Amplitude of all sounds fluctuated throughout each sample. Sounds were normalized in Audacity® 2.22, 2018 using their peak amplitudes, as these determined which period of a sound would be most salient to participants. Peak amplitudes measured above were measured using Bose ${ }^{\circledR}$ On-Ear headphones (Bose Corporation, 2006) and a digital sound level meter (Model SLM01, Tacklife). 
Table 5

Descriptive Statistics of Post-Hoc Analysis of Main Effect for Signal to Noise Ratio

\begin{tabular}{|c|c|c|c|c|c|}
\hline \multirow[b]{2}{*}{$\begin{array}{l}\text { Whistle } \\
\text { Amplitude }\end{array}$} & \multirow[b]{2}{*}{$\begin{array}{l}\text { Noise } \\
\text { Amplitude }\end{array}$} & \multirow{2}{*}{$\begin{array}{l}\text { Mean } \\
\text { Performance } \\
(\%)\end{array}$} & \multicolumn{2}{|c|}{ 95\% Confidence Interval } & \multirow[b]{2}{*}{ Group } \\
\hline & & & $\begin{array}{l}\text { Lower Bound } \\
(\%)\end{array}$ & Upper Bound (\%) & \\
\hline \multirow[t]{4}{*}{ Low } & None & 97.5 & 95.8 & 98.6 & A \\
\hline & Low & 94.0 & 91.8 & 95.6 & $\mathrm{~B}$ \\
\hline & Medium & 78.3 & 73.6 & 82.3 & $\mathrm{C}$ \\
\hline & High & 38.9 & 33.5 & 44.4 & $\mathrm{D}$ \\
\hline \multirow[t]{4}{*}{ Medium } & None & 97.8 & 95.5 & 98.9 & $\mathrm{AB}$ \\
\hline & Low & 96.9 & 94.8 & 98.2 & $\mathrm{AB}$ \\
\hline & Medium & 95.9 & 94.1 & 97.2 & $\mathrm{AB}$ \\
\hline & High & 78.0 & 73.3 & 82.1 & $\mathrm{C}$ \\
\hline
\end{tabular}

Note. This table shows the post-hoc analysis of the main effect for signal to noise ratio. The displayed data were generated using a multilevel logistic regression model (Raundenbush \& Bryk, 2002). An experimental type I error level of $\alpha=0.05$ was used with Tukey's (1949) HSD to correct for multiple comparisons. Conditions where performance was not statistically significantly different share a group, and conditions where performance was statistically significantly different have different groups. 
Table 6

Odds Ratios and Significance Testing from Post-Hoc Analysis of the Main Effect for Signal to

Noise Ratio

\begin{tabular}{|c|c|c|c|c|c|}
\hline \multicolumn{2}{|c|}{ First Signal to Noise Ratio } & \multicolumn{2}{|c|}{ Second Signal to Noise Ratio } & \multirow[b]{3}{*}{ Odds Ratio } & \multirow[b]{3}{*}{$p$ Value } \\
\hline Whistle & Noise & Whistle & Noise & & \\
\hline Amplitude & Amplitude & Amplitude & Amplitude & & \\
\hline \multirow[t]{22}{*}{ Low } & \multirow{7}{*}{ None } & \multirow[t]{3}{*}{ Low } & Low & 2.54 & 0.042 \\
\hline & & & Medium & 10.95 & $<0.001$ \\
\hline & & & High & 62.02 & $<0.001$ \\
\hline & & \multirow[t]{4}{*}{ Medium } & None & 1.11 & 1.000 \\
\hline & & & Low & 1.26 & 0.999 \\
\hline & & & Medium & 1.69 & 0.712 \\
\hline & & & High & 11.11 & $<0.001$ \\
\hline & \multirow[t]{6}{*}{ Low } & \multirow[t]{2}{*}{ Low } & Medium & 4.32 & $<0.001$ \\
\hline & & & High & 24.46 & $<0.001$ \\
\hline & & \multirow[t]{4}{*}{ Medium } & None & $3.56^{*} 10^{-1}$ & 0.112 \\
\hline & & & Low & $4.98 * 10^{-1}$ & 0.252 \\
\hline & & & Medium & $6.67 * 10^{-1}$ & 0.594 \\
\hline & & & High & 4.38 & $<0.001$ \\
\hline & \multirow[t]{5}{*}{ Medium } & Low & High & 5.66 & $<0.001$ \\
\hline & & \multirow[t]{4}{*}{ Medium } & None & $8.23 * 10^{-2}$ & $<0.001$ \\
\hline & & & Low & $1.15 * 10^{-1}$ & $<0.001$ \\
\hline & & & Medium & $1.54 * 10^{-1}$ & $<0.001$ \\
\hline & & & High & 1.01 & 1.000 \\
\hline & \multirow[t]{4}{*}{ High } & \multirow[t]{4}{*}{ Medium } & None & $1.45 * 10^{-2}$ & $<0.001$ \\
\hline & & & Low & $2.03 * 10^{-2}$ & $<0.001$ \\
\hline & & & Medium & $2.73 * 10^{-2}$ & $<0.001$ \\
\hline & & & High & $1.79 * 10^{-1}$ & $<0.001$ \\
\hline \multirow[t]{6}{*}{ Medium } & \multirow[t]{3}{*}{ None } & \multirow[t]{3}{*}{ Medium } & Low & 1.40 & 0.995 \\
\hline & & & Medium & 1.88 & 0.743 \\
\hline & & & High & 12.33 & $<0.001$ \\
\hline & \multirow[t]{2}{*}{ Low } & \multirow[t]{2}{*}{ Medium } & Medium & $7.46^{*} 10^{-1}$ & 0.981 \\
\hline & & & High & 8.81 & $<0.001$ \\
\hline & Medium & Medium & High & 6.57 & $<0.001$ \\
\hline
\end{tabular}

Note. This table shows the post-hoc analysis of the main effect for signal to noise ratio. The displayed data were generated using a multilevel logistic regression model (Raundenbush \& Bryk, 2002). An experimental type I error level of $\alpha=0.05$ was used with Tukey's (1949) HSD to correct for multiple comparisons. 
Table 7

Post-Hoc Analysis of Interaction Effect Between Boat Noise Sample and Signal to Noise Ratio

\begin{tabular}{|c|c|c|c|c|c|c|}
\hline \multirow{3}{*}{$\begin{array}{l}\text { Whistle } \\
\text { Amplitude }\end{array}$} & \multirow{3}{*}{$\begin{array}{l}\text { Noise } \\
\text { Amplitude }\end{array}$} & \multirow{3}{*}{$\begin{array}{l}\text { Boat } \\
\text { Noise } \\
\text { Sample }\end{array}$} & \multirow{3}{*}{$\begin{array}{l}\text { Mean } \\
\text { Performance } \\
(\%)\end{array}$} & \multicolumn{2}{|c|}{$95 \%$ Confidence Interval } & \multirow[t]{3}{*}{ Group } \\
\hline & & & & Lower & Upper & \\
\hline & & & & Bound $(\%)$ & Bound $(\%)$ & \\
\hline \multirow[t]{15}{*}{ Low } & \multirow[t]{5}{*}{ Low } & 1 & 93.1 & 88.8 & 95.8 & A \\
\hline & & 2 & 96.0 & 92.9 & 97.8 & $\mathrm{AB}$ \\
\hline & & 3 & 91.4 & 86.6 & 94.6 & $\mathrm{BC}$ \\
\hline & & 4 & 92.3 & 87.8 & 95.3 & $\mathrm{ABC}$ \\
\hline & & 5 & 89.8 & 84.6 & 93.4 & $\mathrm{C}$ \\
\hline & \multirow[t]{5}{*}{ Medium } & 1 & 93.1 & 88.8 & 95.8 & A \\
\hline & & 2 & 82.3 & 75.2 & 87.6 & $\mathrm{~B}$ \\
\hline & & 3 & 57.4 & 48.6 & 65.8 & $\mathrm{C}$ \\
\hline & & 4 & 65.1 & 56.2 & 73.1 & $\mathrm{C}$ \\
\hline & & 5 & 79.4 & 72.2 & 85.1 & $\mathrm{~B}$ \\
\hline & \multirow[t]{5}{*}{ High } & 1 & 53.5 & 45.0 & 61.9 & $\mathrm{~A}$ \\
\hline & & 2 & 43.4 & 34.8 & 52.4 & $\mathrm{AB}$ \\
\hline & & 3 & 28.0 & 21.3 & 35.9 & $\mathrm{C}$ \\
\hline & & 4 & 30.7 & 23.5 & 38.9 & $\mathrm{BC}$ \\
\hline & & 5 & 40.5 & 32.5 & 49.0 & $\mathrm{ABC}$ \\
\hline \multirow[t]{15}{*}{ Medium } & \multirow[t]{5}{*}{ Low } & 1 & 97.2 & 94.3 & 98.7 & A \\
\hline & & 2 & 97.7 & 95.2 & 98.9 & A \\
\hline & & 3 & 97.8 & 95.2 & 99.0 & A \\
\hline & & 4 & 95.5 & 91.5 & 97.7 & $\mathrm{~A}$ \\
\hline & & 5 & 95.5 & 91.5 & 97.7 & $\mathrm{~A}$ \\
\hline & \multirow[t]{5}{*}{ Medium } & 1 & 97.7 & 95.2 & 98.9 & $\mathrm{~A}$ \\
\hline & & 2 & 96.5 & 93.5 & 98.2 & $\mathrm{AB}$ \\
\hline & & 3 & 91.9 & 87.1 & 95.0 & B \\
\hline & & 4 & 96.4 & 93.2 & 98.1 & $\mathrm{AB}$ \\
\hline & & 5 & 95.1 & 91.4 & 97.2 & $\mathrm{AB}$ \\
\hline & \multirow[t]{5}{*}{ High } & 1 & 92.5 & 88.1 & 95.4 & $\mathrm{~A}$ \\
\hline & & 2 & 86.8 & 80.9 & 91.1 & $\mathrm{AB}$ \\
\hline & & 3 & 48.6 & 40.0 & 57.3 & $\mathrm{C}$ \\
\hline & & 4 & 60.6 & 51.6 & 68.9 & $\mathrm{C}$ \\
\hline & & 5 & 82.6 & 76.0 & 87.7 & B \\
\hline
\end{tabular}

Note. This table shows the post-hoc analysis of the interaction effect between boat noise

sample and signal to noise ratio. The displayed data were generated using a multilevel logistic regression model (Raundenbush \& Bryk, 2002). An experimental type I error level of $\alpha=0.05$ was used with Holm's correction (1979) for multiple hypothesis 
testing. Noises 1-5 were compared within each level of signal to noise ratio; no comparisons were made between levels of signal to noise ratio. Conditions where performance was not statistically significantly different share a group, and conditions where performance was statistically significantly different have different groups. Conditions with no boat noise (low signal with no noise, medium signal with no noise) were not included as there were no noises played during these trials. 
Table 8

Post-Hoc Analysis of Interaction Effect Between Boat Noise Peak Frequency and Signal to Noise Ratio

\begin{tabular}{|c|c|c|c|c|c|c|c|}
\hline \multirow[b]{3}{*}{$\begin{array}{l}\text { Whistle } \\
\text { Amplitude }\end{array}$} & \multirow[b]{3}{*}{$\begin{array}{l}\text { Noise } \\
\text { Amplitude }\end{array}$} & \multirow[b]{3}{*}{$\begin{array}{l}\text { Noise } \\
\text { Sample }\end{array}$} & \multirow{3}{*}{$\begin{array}{l}\text { Noise Peak } \\
\text { Frequency } \\
(\mathrm{kHz})\end{array}$} & \multirow{3}{*}{$\begin{array}{l}\text { Mean } \\
\text { Performance } \\
(\%)\end{array}$} & \multicolumn{2}{|c|}{$\begin{array}{l}\text { 95\% Confidence } \\
\text { Interval }\end{array}$} & \multirow{3}{*}{ Group } \\
\hline & & & & & Lower & Upper & \\
\hline & & & & & $\begin{array}{l}\text { Bound } \\
(\%)\end{array}$ & $\begin{array}{l}\text { Bound } \\
(\%)\end{array}$ & \\
\hline \multirow[t]{10}{*}{ Low } & \multirow[t]{5}{*}{ Medium } & 1 & 0.063 & 86.5 & 81.8 & 90.1 & A \\
\hline & & 4 & 0.132 & 77.8 & 73.4 & 81.6 & B \\
\hline & & 5 & 0.166 & 72.2 & 67.7 & 76.4 & $\mathrm{C}$ \\
\hline & & 2 & 0.186 & 68.6 & 63.6 & 73.2 & $\mathrm{D}$ \\
\hline & & 3 & 0.233 & 59.2 & 52.3 & 65.7 & $\mathrm{E}$ \\
\hline & \multirow[t]{5}{*}{ High } & 1 & 0.063 & 50.2 & 43.0 & 57.3 & A \\
\hline & & 4 & 0.132 & 42.5 & 37.4 & 47.7 & B \\
\hline & & 5 & 0.166 & 38.8 & 34.0 & 43.9 & $\mathrm{C}$ \\
\hline & & 2 & 0.186 & 36.7 & 31.8 & 42.0 & $\mathrm{D}$ \\
\hline & & 3 & 0.233 & 32.0 & 26.4 & 38.2 & $\mathrm{E}$ \\
\hline \multirow[t]{10}{*}{ Medium } & \multirow[t]{5}{*}{ Medium } & 1 & 0.063 & 96.9 & 94.6 & 98.2 & $\mathrm{~A}$ \\
\hline & & 4 & 0.132 & 95.2 & 93.3 & 96.6 & B \\
\hline & & 5 & 0.166 & 94.0 & 92.1 & 95.5 & $\mathrm{C}$ \\
\hline & & 2 & 0.186 & 93.3 & 91.0 & 95.0 & $\mathrm{D}$ \\
\hline & & 3 & 0.233 & 91.0 & 87.1 & 93.8 & $\mathrm{E}$ \\
\hline & \multirow[t]{5}{*}{ High } & 1 & 0.063 & 86.4 & 81.8 & 90.0 & A \\
\hline & & 4 & 0.132 & 77.0 & 72.6 & 80.9 & B \\
\hline & & 5 & 0.166 & 70.9 & 66.3 & 75.1 & $\mathrm{C}$ \\
\hline & & 2 & 0.186 & 66.9 & 61.9 & 71.6 & $\mathrm{D}$ \\
\hline & & 3 & 0.233 & 56.6 & 49.8 & 63.2 & $\mathrm{E}$ \\
\hline
\end{tabular}

Note. This table shows the interaction effect between noise peak frequency and signal to noise ratio. The displayed data were generated using a multilevel logistic regression model (Raundenbush \& Bryk, 2002), after controlling for other variables. An experimental type I error level of $\alpha=0.05$ was used with Holm's correction (1979) for multiple hypothesis testing. Conditions where performance was not statistically significantly different share a group, and conditions where performance was statistically significantly different have different groups. The following signal to noise conditions are not included and had no statistically significant, 
within-group differences: low signal and no noise, low signal and low noise, medium signal and no noise, and medium signal and low noise conditions. 
Table 9

Post-Hoc Analysis of Interaction Effect Between Noise Peak Frequency and Whistle Peak

Frequency

\begin{tabular}{|c|c|c|c|c|c|c|c|}
\hline \multirow[b]{3}{*}{$\begin{array}{l}\text { Noise } \\
\text { Sample }\end{array}$} & \multirow{3}{*}{$\begin{array}{l}\text { Noise } \\
\text { Peak } \\
\text { Frequency } \\
(\mathrm{kHz})\end{array}$} & \multirow[b]{3}{*}{ Dolphin } & \multirow{3}{*}{$\begin{array}{l}\text { Whistle } \\
\text { Peak } \\
\text { Frequency } \\
(\mathrm{kHz})\end{array}$} & \multirow{3}{*}{$\begin{array}{l}\text { Mean } \\
\text { Performance } \\
(\%)\end{array}$} & \multicolumn{2}{|c|}{$\begin{array}{l}95 \% \text { Confidence } \\
\text { Interval }\end{array}$} & \multirow[b]{3}{*}{ Group } \\
\hline & & & & & Lower & Upper & \\
\hline & & & & & $\begin{array}{l}\text { Bound } \\
(\%)\end{array}$ & $\begin{array}{l}\text { Bound } \\
(\%)\end{array}$ & \\
\hline \multirow[t]{3}{*}{1} & 0.063 & $\mathrm{C}$ & 7.07 & 92.2 & 89.8 & 94.0 & $\mathrm{~A}$ \\
\hline & & $\mathrm{A}$ & 8.68 & 91.8 & 89.9 & 93.3 & $\mathrm{~A}$ \\
\hline & & B & 10.05 & 91.4 & 88.9 & 93.4 & $\mathrm{~A}$ \\
\hline \multirow[t]{3}{*}{4} & 0.132 & $\mathrm{C}$ & 7.07 & 87.6 & 85.1 & 89.7 & A \\
\hline & & $\mathrm{A}$ & 8.68 & 88.8 & 86.8 & 90.5 & A \\
\hline & & B & 10.05 & 89.7 & 87.6 & 91.5 & A \\
\hline \multirow[t]{3}{*}{5} & 0.166 & $\mathrm{C}$ & 7.07 & 84.6 & 81.7 & 87.0 & A \\
\hline & & A & 8.68 & 87.0 & 84.8 & 88.9 & B \\
\hline & & B & 10.05 & 88.8 & 86.6 & 90.7 & $\mathrm{C}$ \\
\hline \multirow[t]{3}{*}{2} & 0.186 & $\mathrm{C}$ & 7.07 & 82.5 & 79.4 & 85.3 & A \\
\hline & & $\mathrm{A}$ & 8.68 & 86.8 & 83.5 & 87.9 & $\mathrm{~B}$ \\
\hline & & B & 10.05 & 88.3 & 85.9 & 90.3 & $\mathrm{C}$ \\
\hline \multirow[t]{3}{*}{3} & 0.233 & $\mathrm{C}$ & 7.07 & 76.9 & 72.4 & 80.9 & A \\
\hline & & A & 8.68 & 82.8 & 79.7 & 85.5 & B \\
\hline & & B & 10.05 & 86.8 & 83.7 & 89.4 & $\mathrm{C}$ \\
\hline
\end{tabular}

Note. This table shows the interaction effect between noise peak frequency and whistle peak

frequency. The displayed data were generated using a multilevel logistic regression model

(Raundenbush \& Bryk, 2002), after controlling for other variables. An experimental type I error

level of $\alpha=0.05$ was used with Holm's correction (1979) for multiple hypothesis testing.

Conditions where performance was not statistically significantly different share a group, and

conditions where performance was statistically significantly different have different groups. The

peak frequencies of dolphin whistle $\mathrm{A}, \mathrm{B}$, and $\mathrm{C}$ were compared within each level of peak noise

frequency; no comparisons were made between samples of noise. 
Table 10

Cues Reported Most Useful for Each Dolphin in Experiment 2

\begin{tabular}{|c|c|c|c|c|c|c|c|c|}
\hline \multirow[t]{2}{*}{ Whistle cues } & \multicolumn{4}{|c|}{$\begin{array}{l}\text { Number of times reported used without } \\
\text { noise per dolphin }\end{array}$} & \multicolumn{4}{|c|}{$\begin{array}{l}\text { Number of times reported used with } \\
\text { noise per dolphin }\end{array}$} \\
\hline & $\begin{array}{l}\text { Dolphin } \\
\text { A }\end{array}$ & $\begin{array}{l}\text { Dolphin } \\
\text { B }\end{array}$ & $\begin{array}{l}\text { Dolphin } \\
\text { C }\end{array}$ & Sum & $\begin{array}{l}\text { Dolphin } \\
\text { A }\end{array}$ & $\begin{array}{l}\text { Dolphin } \\
\text { B }\end{array}$ & $\begin{array}{l}\text { Dolphin } \\
\text { C }\end{array}$ & Sum \\
\hline Amplitude & 1 & 0 & 3 & 4 & 3 & 0 & 3 & 6 \\
\hline Frequency & 2 & 3 & 6 & 11 & 7 & 6 & 4 & 17 \\
\hline Frequency contour & 23 & 33 & 26 & 82 & 22 & 31 & 27 & 80 \\
\hline Duration & 24 & 10 & 17 & 51 & 19 & 12 & 20 & 51 \\
\hline Timbre & 0 & 2 & 11 & 13 & 0 & 2 & 8 & 10 \\
\hline Other & 0 & 0 & 0 & 0 & 0 & 1 & 1 & 2 \\
\hline Sum & 50 & 48 & 63 & 161 & 51 & 52 & 63 & 166 \\
\hline
\end{tabular}

Note. Participants $(\mathrm{N}=36)$ were allowed to select one or two cues per dolphin. They

were encouraged to give a single cue if there was a specific cue which was clearly the

most useful for identifying the dolphin in question, or to give two cues if there were

multiple cues that were used to identify that dolphin. 
Table 11

Reported Cue Use in Experiment 2

\begin{tabular}{llll}
\hline Cue & Mean Use & Standard Deviation & Group \\
\hline Amplitude & 2.06 & 1.32 & $\mathrm{D}$ \\
Frequency & 3.51 & 1.80 & $\mathrm{C}$ \\
Frequency Contour & 6.57 & 0.87 & $\mathrm{~A}$ \\
Duration & 5.43 & 1.71 & $\mathrm{~B}$ \\
Timbre & 3.40 & 1.90 & $\mathrm{C}$ \\
Other & 1.57 & 1.35 & $\mathrm{D}$ \\
\hline
\end{tabular}

Note. For each cue, participants were asked to give a number from one to seven, where one would indicate that they never used the cue in question, four would indicate that they used it in about half of the trials, and seven would indicate that they used it in every trial. This table shows the mean reported cue use by participants in the interview phase of Experiment 2. An experimental type I error level of $\alpha=0.05$ was used with a Bonferroni correction for multiple hypothesis testing. Conditions where reported cue use was not statistically significantly different share a group, and conditions where reported cue use was statistically significantly different have different groups. 
Table 12

Post-Hoc Analysis of Interaction Effect Between Reported Cue Use and Presence or Absence of Noise

\begin{tabular}{|c|c|c|c|c|}
\hline Cue & $\begin{array}{l}\text { Noise Present } \\
\text { or Absent }\end{array}$ & Reported Use & $\begin{array}{l}\text { Standard } \\
\text { Deviation }\end{array}$ & Group \\
\hline \multirow[t]{2}{*}{ Amplitude } & Absent & 1.72 & 0.97 & $\mathrm{~B}$ \\
\hline & Present & 2.39 & 1.53 & A \\
\hline \multirow[t]{2}{*}{ Frequency } & Absent & 3.53 & 1.81 & $\mathrm{~A}$ \\
\hline & Present & 3.50 & 1.81 & A \\
\hline \multirow[t]{2}{*}{ Frequency Contour } & Absent & 6.58 & 0.65 & $\mathrm{~A}$ \\
\hline & Present & 6.36 & 1.05 & A \\
\hline \multirow[t]{2}{*}{ Duration } & Absent & 5.56 & 1.58 & A \\
\hline & Present & 5.31 & 1.85 & $\mathrm{~A}$ \\
\hline \multirow[t]{2}{*}{ Timbre } & Absent & 3.36 & 1.79 & $\mathrm{~A}$ \\
\hline & Present & 3.44 & 2.02 & A \\
\hline \multirow[t]{2}{*}{ Other } & Absent & 1.53 & 1.32 & A \\
\hline & Present & 1.61 & 1.40 & $\mathrm{~A}$ \\
\hline
\end{tabular}

Note. For each cue, participants were asked to give a number from one to seven, where one would indicate that they never used the cue in question, four would indicate that they used it in about half of the trials, and seven would indicate that they used it in every trial. This table shows the interaction effect between reported cue use and the presence or absence of boat noise. The data were analyzed using a 2 (absence or presence of noise) $\times 6$ (auditory cue) repeated measures analysis of variance (ANOVA with an experimental type I error level of $\alpha$ $=0.05$ and a Bonferroni correction for multiple hypothesis testing. Reported cue use in the presence or absence of noise was compared for each cue, and comparisons were not made between cues. Conditions where reported cue use was not statistically significantly different share a group, and conditions where reported cue use was statistically significantly different have different groups. 

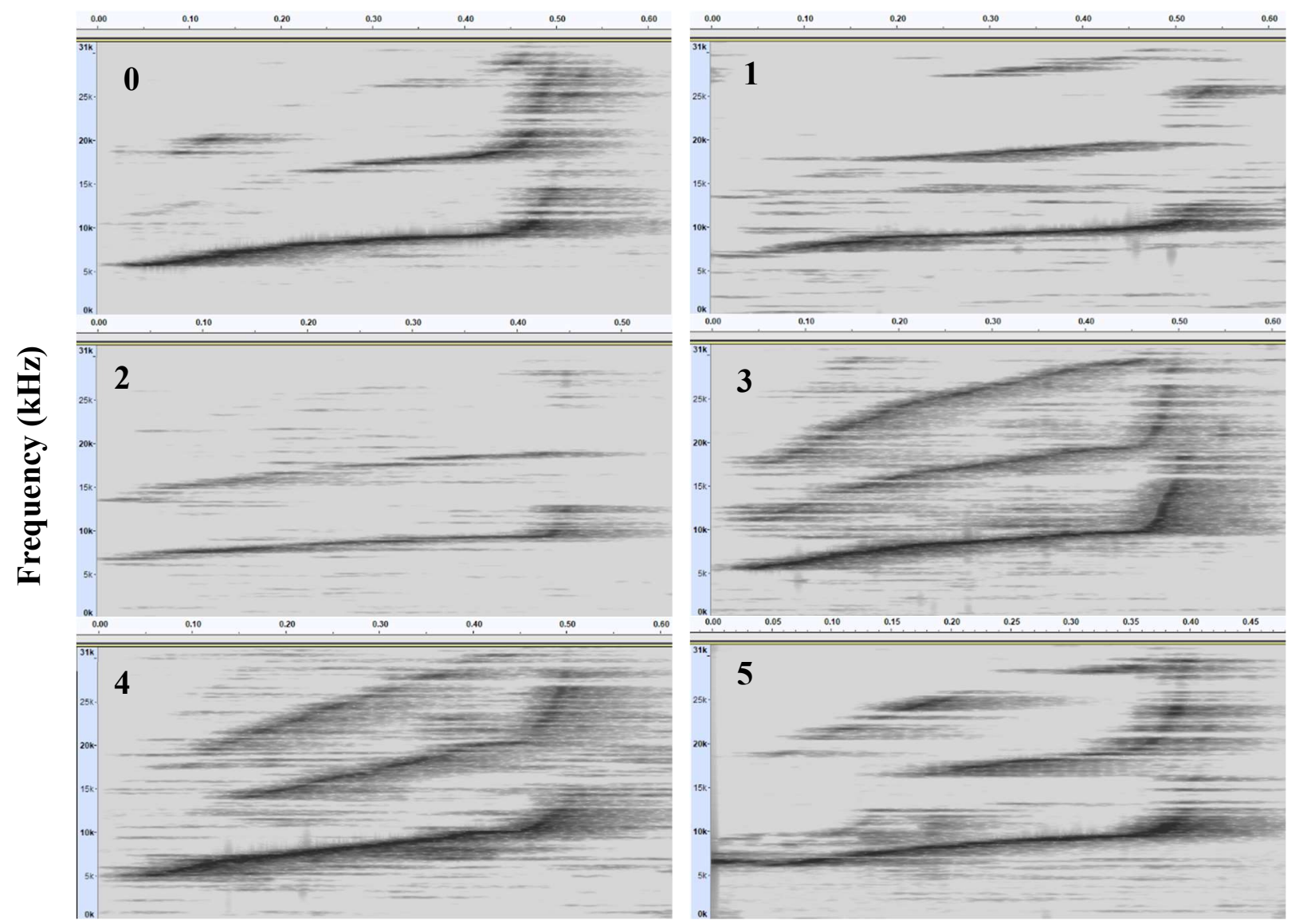

Time (s)

Figure 1. These spectrograms show the five exemplars of Dolphin A's signature whistle used during the experiments. Exemplar 0 was used for training, and exemplars 1-5 were used during the listening tasks. Dolphin A's signature whistle consisted of a segment with relatively flat frequency followed by an upsweep. 


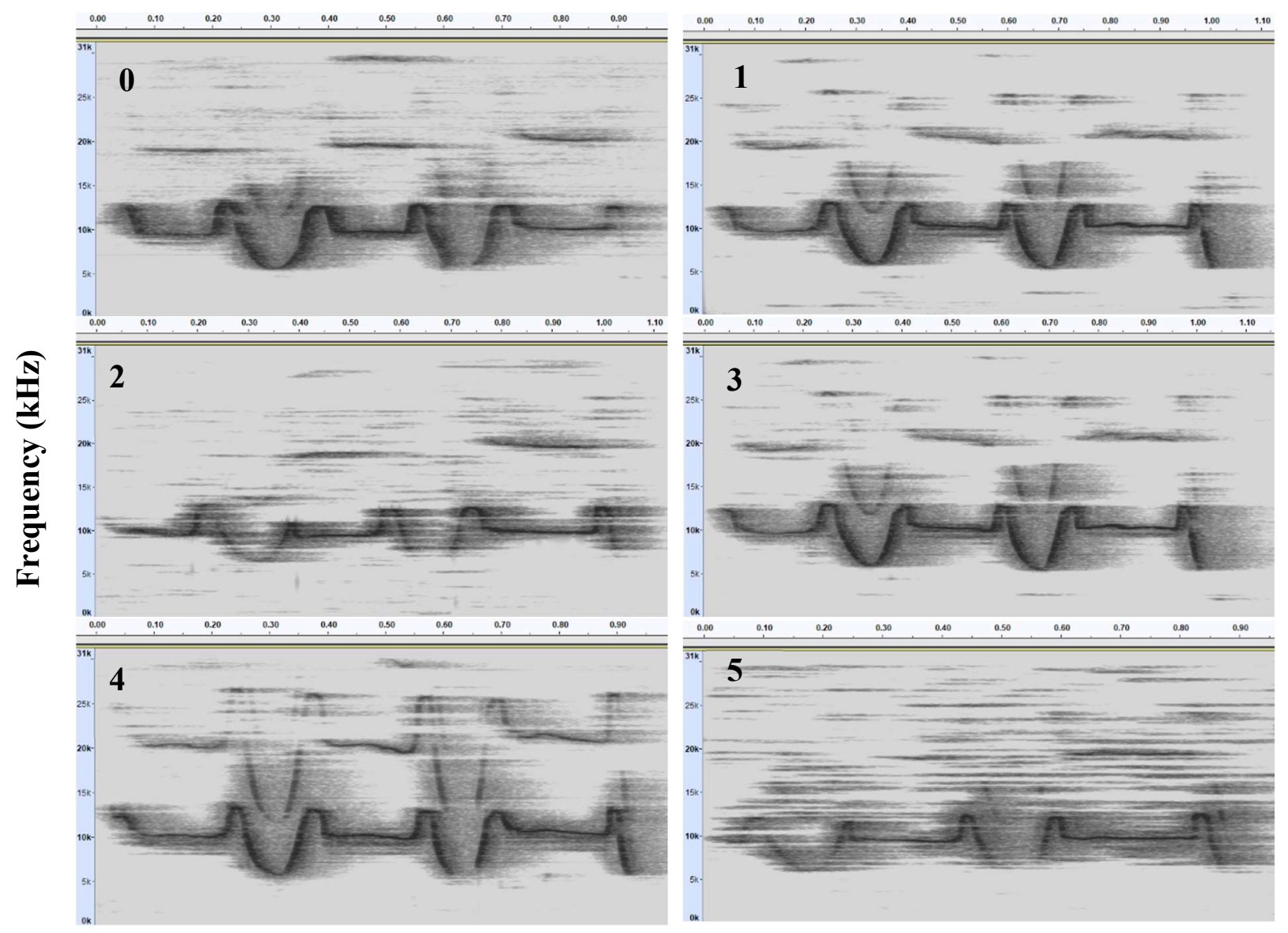

Time (s)

Figure 2. These spectrograms show the frequency contour of Dolphin B's signature whistle over the duration of the whistle. Exemplar 0 was used for training, and exemplars 1-5 were used during the listening tasks. Dolphin B's signature whistle contained segments of relatively constant frequency interspersed with segments with downsweep followed by a symmetrical upsweep. 

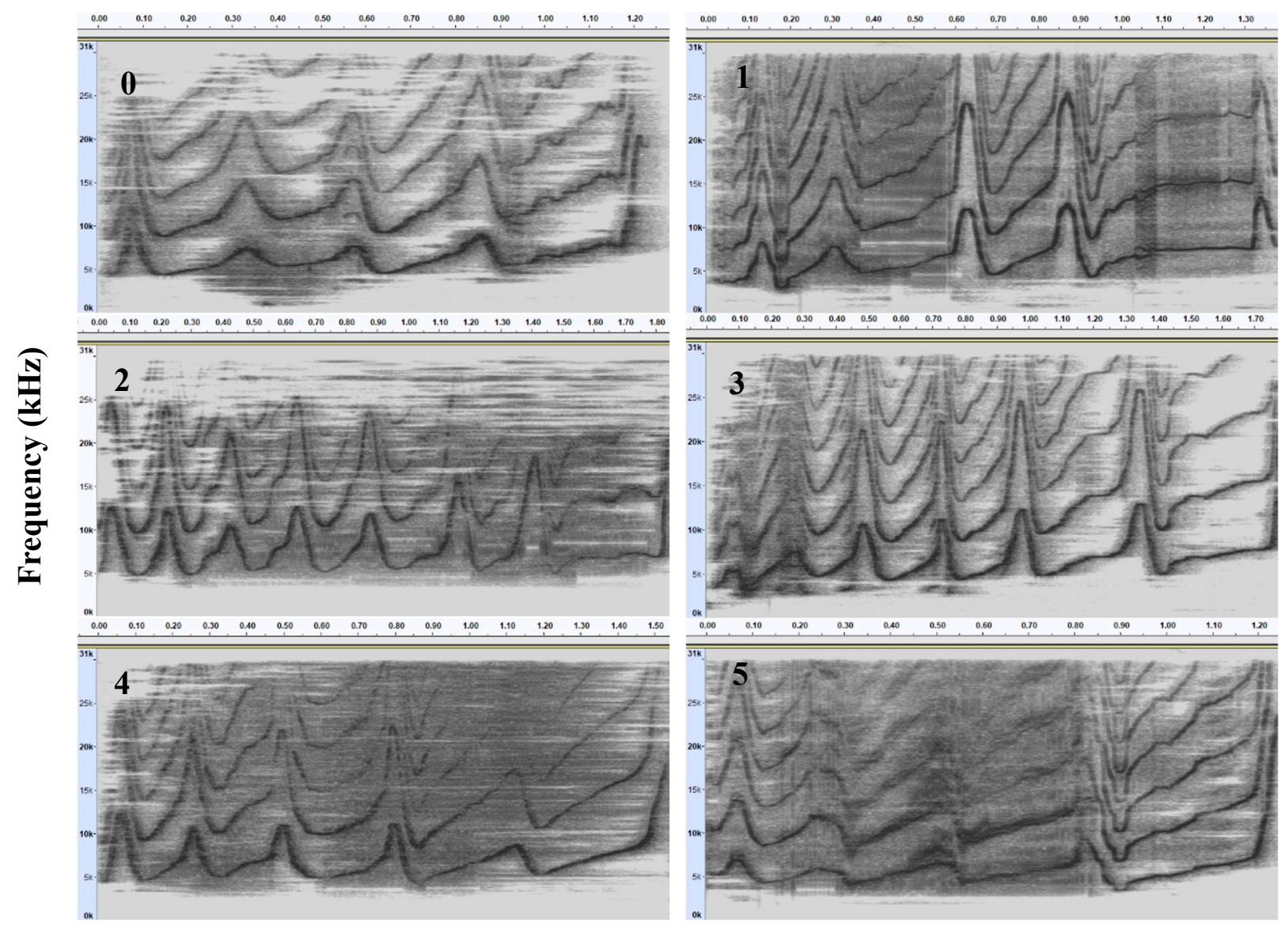

Time (s)

Figure 3. These spectrograms show the frequency contour of Dolphin C's signature whistle over the duration of the whistle. Exemplar 0 was used for training, and exemplars 1-5 were used during the listening tasks. The whistle contains repeated sequences (loops) which are all similar in frequency contour and duration, but the overall number of these loops are not the same for every sample of Dolphin C's signature whistle, so the samples of this signature whistle were not always of the same duration. Note the difference in the number of loops in exemplar 1 and exemplar 3. 


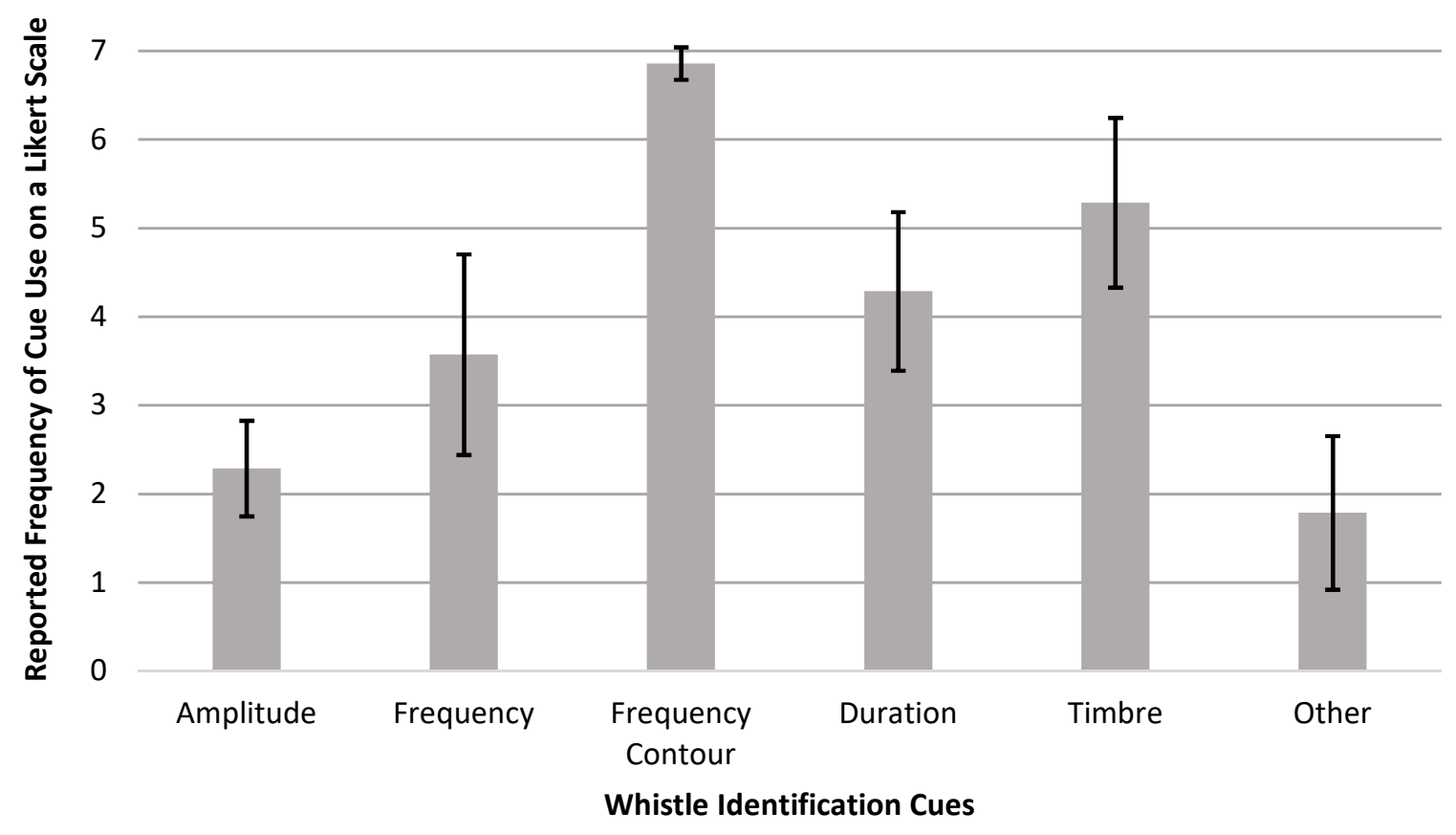

Figure 4. This bar graph shows participants' ratings of how frequently they used each auditory cue to help identify the whistle in a given trial in Experiment 1. For each cue, participants were asked to give a number from one to seven, where one would indicate that they never used the cue in question, four would indicate that they used it in about half of the trials, and seven would indicate that they used it for every trial. Error bars indicate $95 \%$ confidence intervals. 

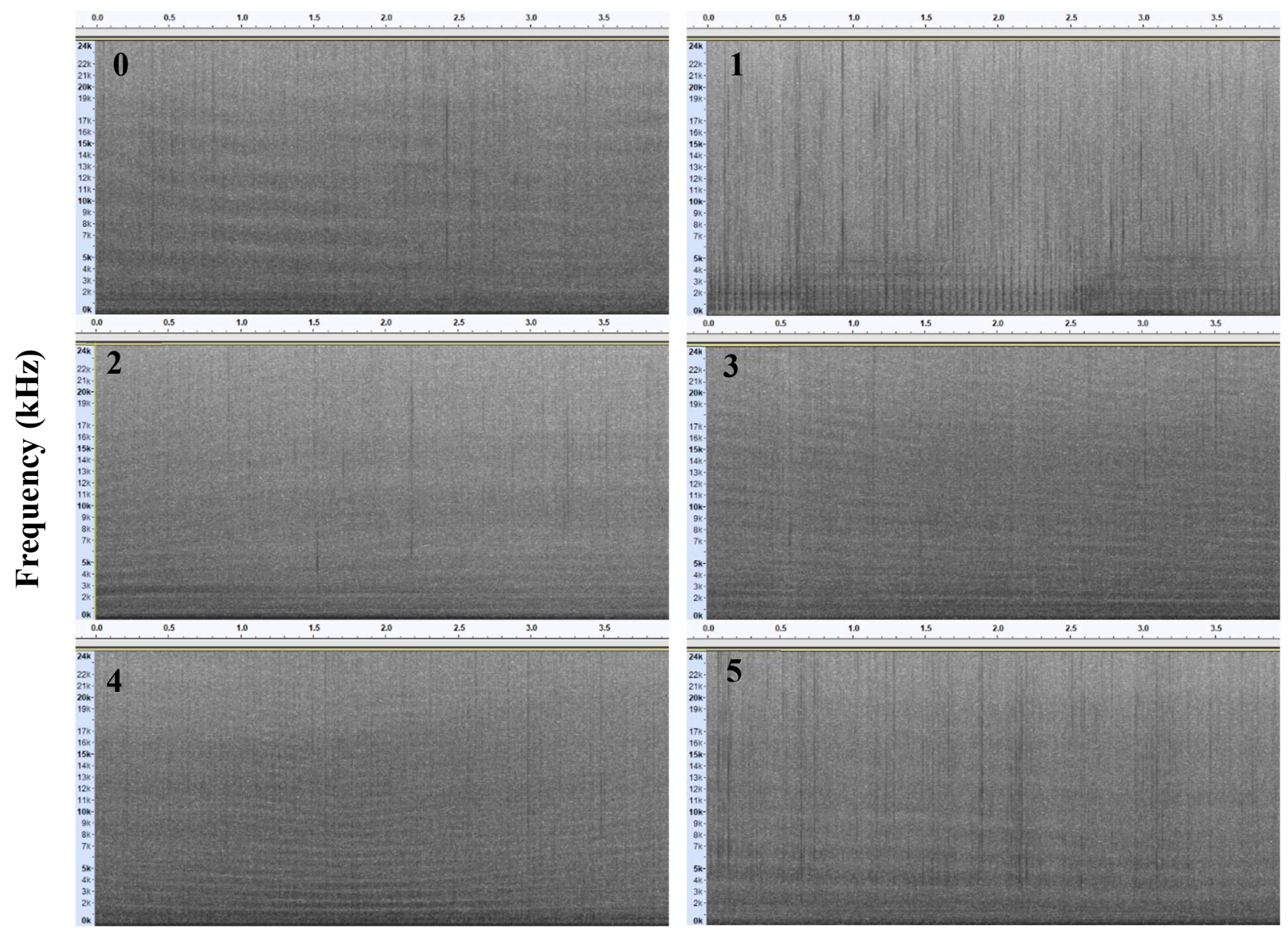

Time (s)

Figure 5. These spectrograms show the six samples of boat noise. Noise 0 was used for an example of boat noise in the interview, and noises 1-5 were used during the listening tasks.

Noises were generally broad-spectrum, continuous sounds. Some samples also had intermittent or repeated broadband sounds in quick succession, with noise 1 containing these features most noticeably of the samples. 

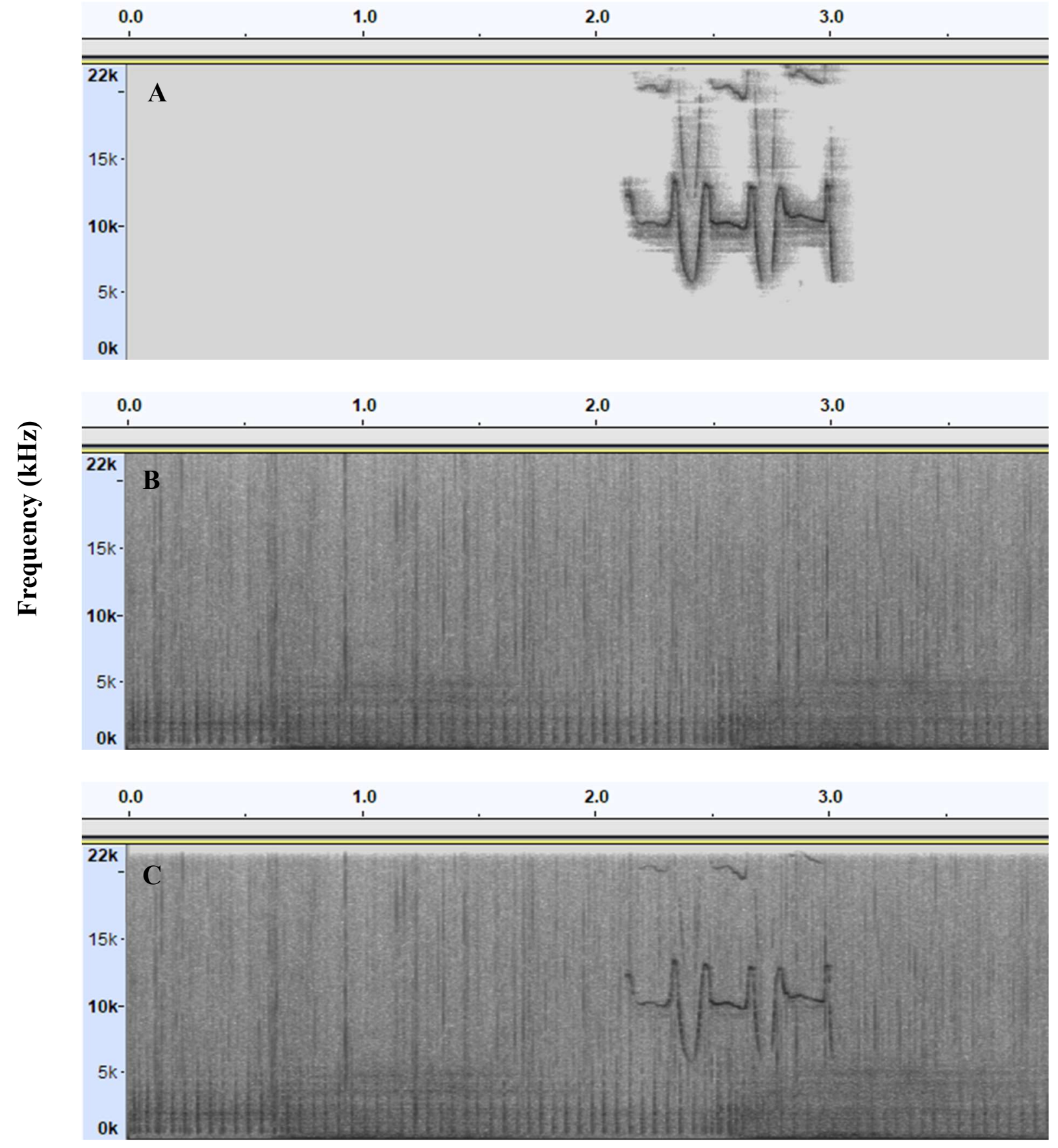

Time (s)

Figure 6. These spectrograms show a sample signature whistle (A), a sample of boat noise (B), and then the two samples merged into a single sound file (C), with both the whistle and the boat noise at their original amplitudes. 


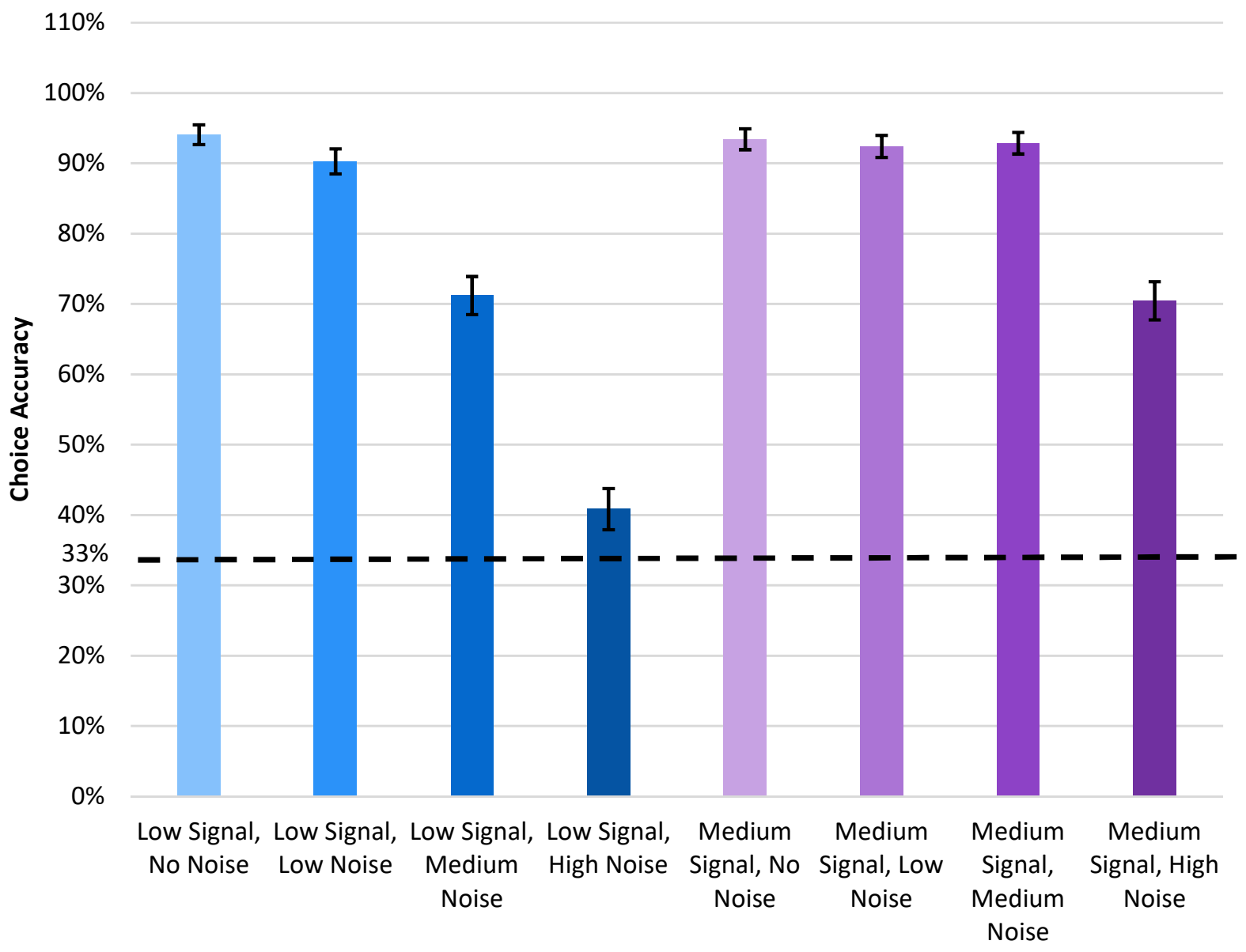

Noise Conditions

Figure 7. This bar graph shows participant average performance at different levels of signal and noise in Experiment 2. Chance performance was 33\% accuracy. Error bars are 95\% confidence intervals. 


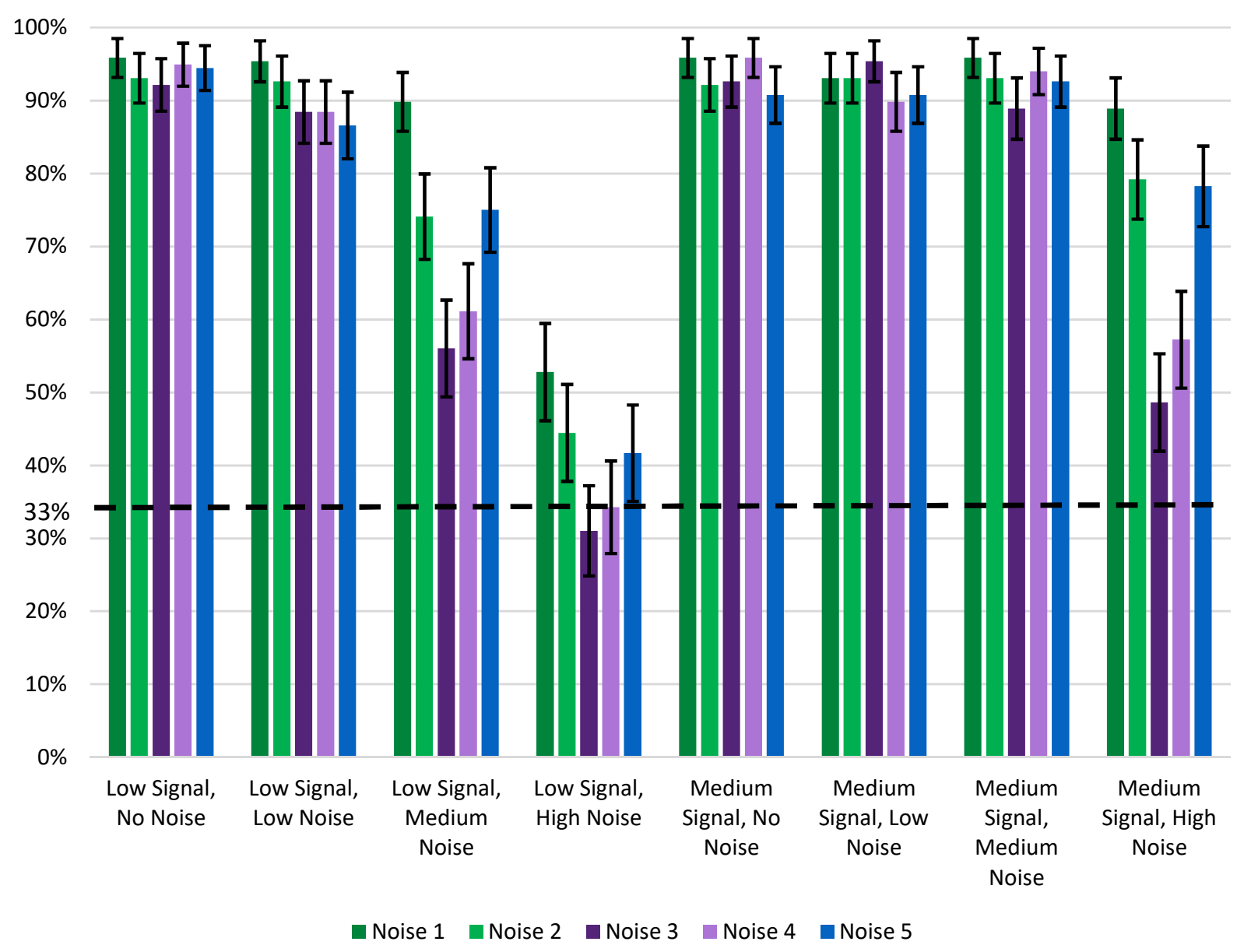

Figure 8. This bar graph shows participant average performance at different levels of signal and noise in Experiment 2, shown across the five samples of boat noise. Chance performance was $33 \%$ accuracy. Error bars are $95 \%$ confidence intervals. 


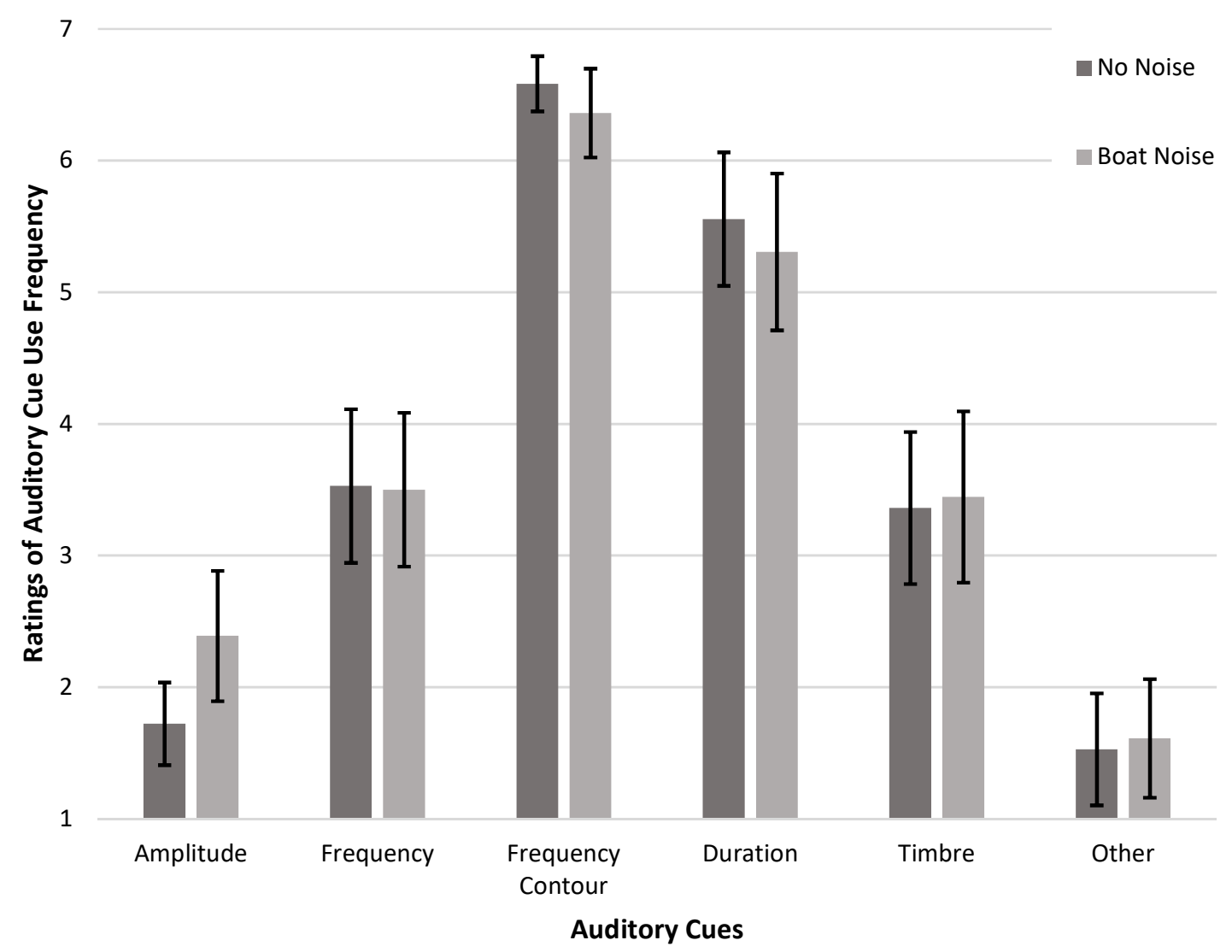

Figure 9. This bar graph shows participants' ratings of how frequently they used each auditory cue to help identify the whistle in a given trial in Experiment 2. For each cue, participants were asked to give a number from one to seven, where one would indicate that they never used the cue in question, four would indicate that they used it in about half of the trials, and seven would indicate that they used it for every trial. Error bars indicate $95 \%$ confidence intervals. 


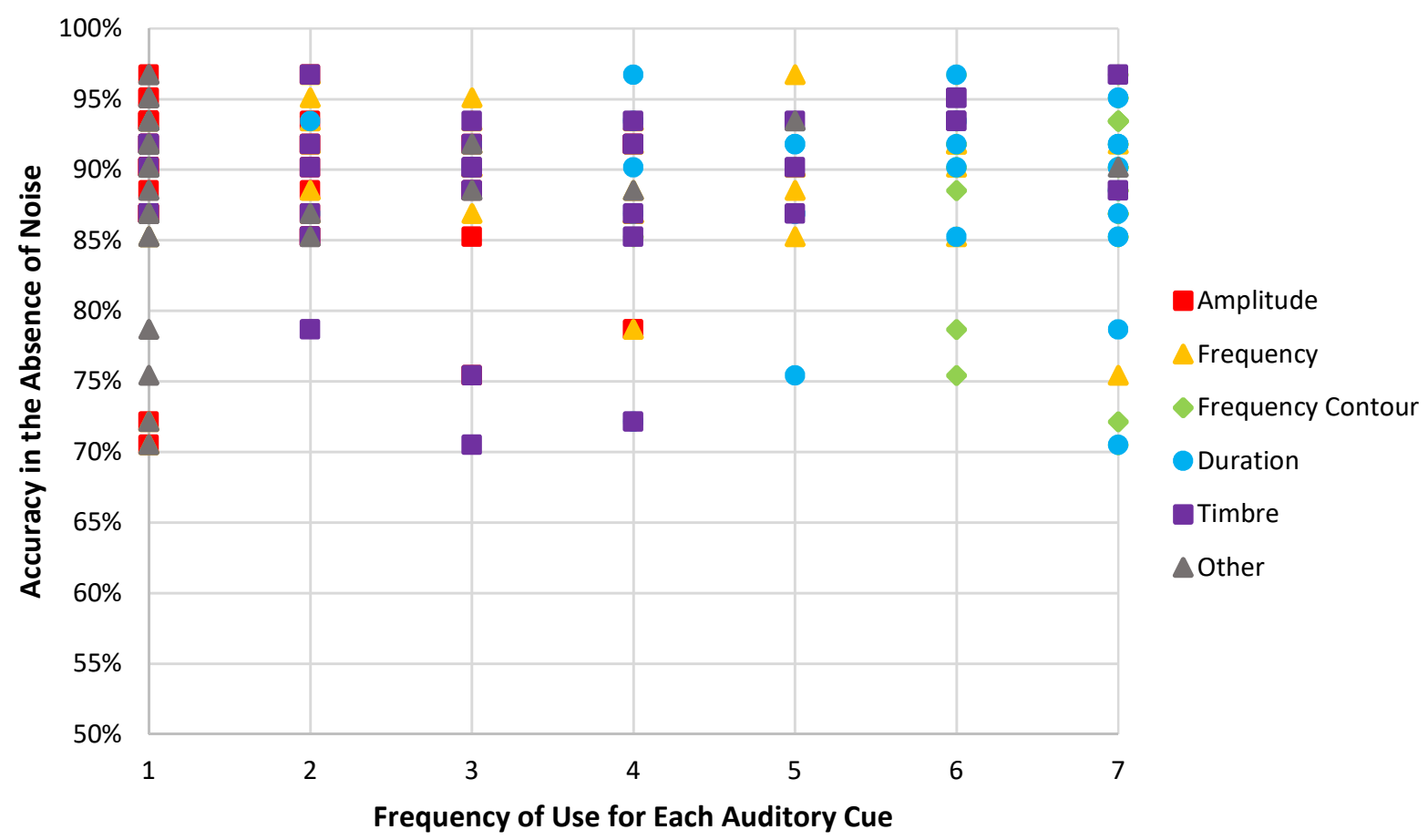

Figure 10. Participant accuracies in Experiment 2 in the absence of noise, compared to participants' ratings of how often they used each auditory cue in the absence of noise. Each point represents one participant's accuracy in the absence of noise on the y-axis, and how frequently they claimed to use a given cue on the x-axis. Some points are be covered by overlapping instances of data. No significant relationships were found between participants' strategies and performance. 


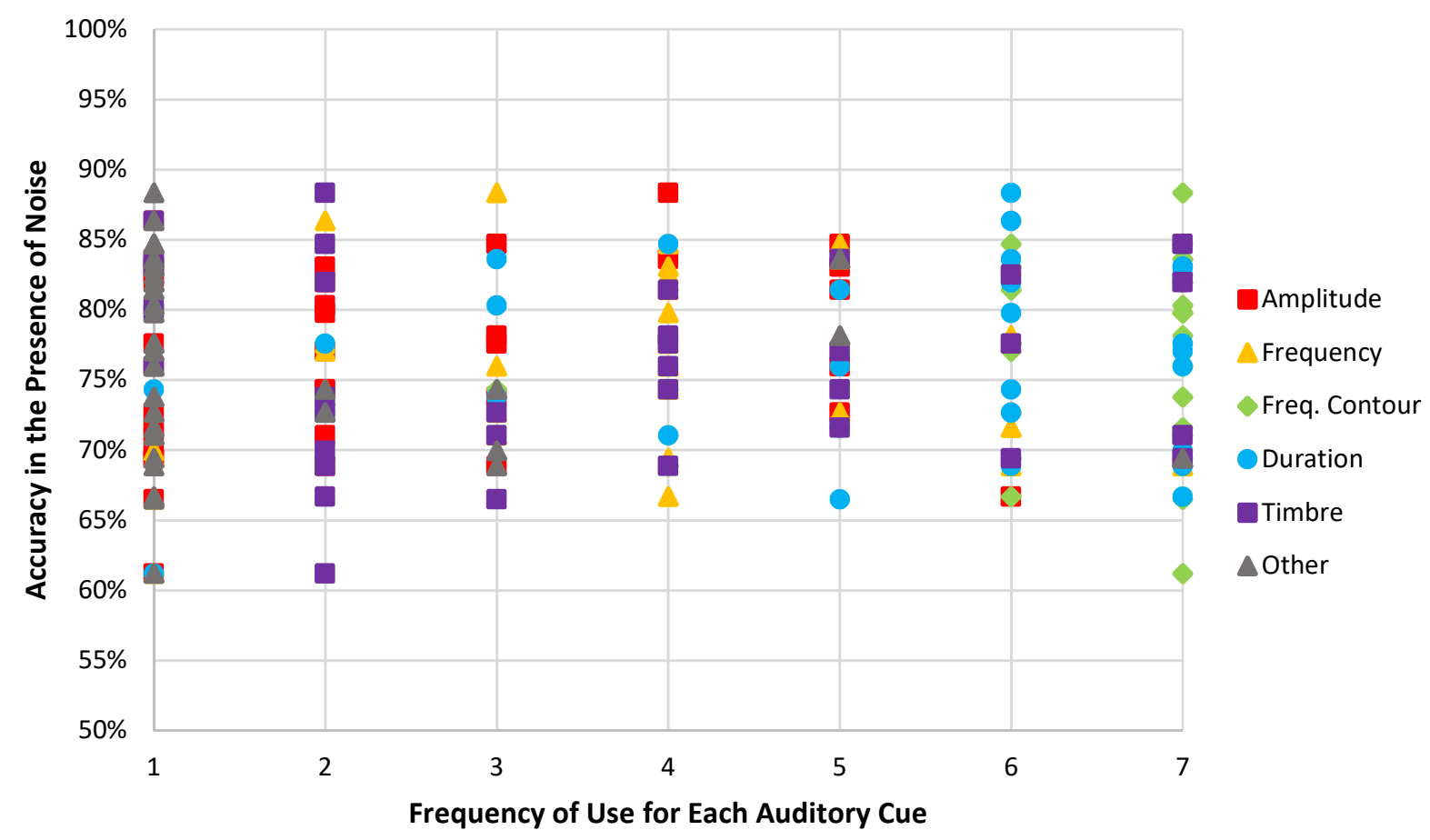

Figure 11. Participant accuracies in Experiment 2 in the presence of noise, compared to participants' ratings of how often they used each auditory cue in the absence of noise. Each point represents one participant's accuracy in the absence of noise on the y-axis, and how frequently they claimed to use a given cue on the x-axis. Some points are be covered by overlapping instances of data. No significant relationships were found between participants' strategies and performance. 